\title{
Experimental mathematics on the magnetic susceptibility of the square lattice Ising model
}

\author{
S. Boukraa ${ }^{\dagger}$, A. J. Guttmann ${ }^{\ddagger}$, S. Hassani ${ }^{\S}$, I. Jensen ${ }^{\ddagger}$, \\ J.-M. Maillard ${ }^{\|}$, B. Nickel ${ }^{+}$and N. Zenine ${ }^{\S}$ \\ $\nmid$ LPTHIRM and Département d'Aéronautique, Université de Blida, Algeria \\ $\ddagger$ ARC Centre of Excellence for Mathematics and Statistics of Complex Systems \\ Department of Mathematics and Statistics, The University of Melbourne, \\ Victoria 3010, Australia \\ $\S$ Centre de Recherche Nucléaire d'Alger, 2 Bd. Frantz Fanon, BP 399, 16000 \\ Alger, Algeria \\ || LPTMC, Université de Paris, Tour 24, 4ème étage, case 121, 4 Place Jussieu, \\ 75252 Paris Cedex 05, France \\ + Department of Physics, University of Guelph, Guelph, Ontario N1G 2W1, \\ Canada \\ E-mail: maillard@lptmc.jussieu.fr, maillard@lptl.jussieu.fr, \\ tonyg@ms.unimelb.edu.au, I. Jensen@ms.unimelb.edu.au, \\ njzenine@yahoo.com, boukraa@mail.univ-blida.dz
}

\begin{abstract}
We calculate very long low- and high-temperature series for the susceptibility $\chi$ of the square lattice Ising model as well as very long series for the five-particle contribution $\chi^{(5)}$ and six-particle contribution $\chi^{(6)}$. These calculations have been made possible by the use of highly optimized polynomial time modular algorithms and a total of more than 150000 CPU hours on computer clusters. The series for $\chi$ (low- and high-temperature regime), $\chi^{(5)}$ and $\chi^{(6)}$ are now extended to 2000 terms. In addition, for $\chi^{(5)}, 10000$ terms of the series are calculated modulo a single prime, and have been used to find the linear ODE satisfied by $\chi^{(5)}$ modulo a prime.

A diff-Padé analysis of the 2000 terms series for $\chi^{(5)}$ and $\chi^{(6)}$ confirms to a very high degree of confidence previous conjectures about the location and strength of the singularities of the $n$-particle components of the susceptibility, up to a small set of "additional" singularities. The exponents at all the singularities of the Fuchsian linear ODE of $\chi^{(5)}$ and the (as yet unknown) ODE of $\chi^{(6)}$ are given: they are all rational numbers. We find the presence of singularities at $w=1 / 2$ for the linear ODE of $\chi^{(5)}$, and $w^{2}=1 / 8$ for the ODE of $\chi^{(6)}$, which are not singularities of the "physical" $\chi^{(5)}$ and $\chi^{(6)}$, that is to say the series-solutions of the ODE's which are analytic at $w=0$.

Furthermore, analysis of the long series for $\chi^{(5)}\left(\right.$ and $\left.\chi^{(6)}\right)$ combined with the corresponding long series for the full susceptibility $\chi$ yields previously conjectured singularities in some $\chi^{(n)}, n \geq 7$. The exponents at all these singularities are also seen to be rational numbers.

We also present a mechanism of resummation of the logarithmic singularities of the $\chi^{(n)}$ leading to the known power-law critical behaviour occurring in the full $\chi$, and perform a power spectrum analysis giving strong arguments in favor of the existence of a natural boundary for the full susceptibility $\chi$.
\end{abstract}

PACS: 05.50.+q, 05.10.-a, 02.30.Hq, 02.30.Gp, 02.40.Xx

AMS Classification scheme numbers: 34M55, 47E05, 81Qxx, 32G34, 34Lxx, $34 \mathrm{Mxx}, 14 \mathrm{Kxx}$ 
Key-words: Susceptibility of the Ising model, long series expansions, singular behavior, diff-Padé series analysis, modular formal calculations, pinch singularities, holonomic functions, multivalued functions, Fuchsian linear differential equations, indicial polynomials, rational critical exponents, holonomy theory, apparent singularities, natural boundary, fast-Fourier transform.

\section{Introduction}

The magnetic susceptibility of the two-dimensional Ising model has attracted the attention of many of the mathematicians and physicists who work (or worked) in the area of equilibrium statistical mechanics for more than 60 years, ever since Onsager's celebrated solution of the free-energy of the two-dimensional Ising model [1].

Let $\sigma_{i, j}$ be the spin at lattice site $(i, j)$. The two-point correlation function is defined as

$$
C(M, N)=\left\langle\sigma_{0,0} \sigma_{M, N}\right\rangle,
$$

and the magnetic susceptibility is given by

$$
k T \cdot \chi=\sum_{M} \sum_{N}\left(C(M, N)-\mathcal{M}^{2}\right),
$$

where the magnetisation $\mathcal{M}$ is zero for $T>T_{c}$ and, in the isotropic case, $\mathcal{M}=$ $\left(1-s^{-4}\right)^{1 / 8}$ for $T<T_{c}$ (in the scaling limit $M, N \rightarrow \infty$ ), where $s=\sinh (2 J / k T)$.

To this day, while we still lack a closed-form solution for the susceptibility, we do have a great deal of associated knowledge. While there have been many breakthroughs in the study of this problem, we would like to mention five in particular.

Firstly, in 1976, Wu, McCoy, Tracy and Barouch [2] showed that the susceptibility can be expressed as an infinite sum of contributions, known as n-particle contributions, so that the high-temperature susceptibility is given by

$$
k T \cdot \chi_{H}(w)=\sum \chi^{(2 n+1)}(w)=\frac{1}{s} \cdot\left(1-s^{4}\right)^{\frac{1}{4}} \cdot \sum \tilde{\chi}^{(2 n+1)}(w)
$$

and the low-temperature susceptibility is given by

$$
k T \cdot \chi_{L}(w)=\sum \chi^{(2 n)}(w)=\left(1-1 / s^{4}\right)^{\frac{1}{4}} \cdot \sum \tilde{\chi}^{(2 n)}(w)
$$

in terms of the self-dual temperature variable $w=\frac{1}{2} s /\left(1+s^{2}\right)$.

As is now well known [2], the $n$-particle contributions have an integral representation and are given by the $(n-1)$-dimensional integrals $[3,4,5,6]$,

$$
\tilde{\chi}^{(n)}(w)=\frac{1}{n !} \cdot\left(\prod_{j=1}^{n-1} \int_{0}^{2 \pi} \frac{d \phi_{j}}{2 \pi}\right)\left(\prod_{j=1}^{n} y_{j}\right) \cdot R^{(n)} \cdot\left(G^{(n)}\right)^{2},
$$

where§

$$
G^{(n)}=\prod_{1 \leq i<j \leq n} h_{i j}, \quad h_{i j}=\frac{2 \sin \left(\left(\phi_{i}-\phi_{j}\right) / 2\right) \cdot \sqrt{x_{i} x_{j}}}{1-x_{i} x_{j}},
$$

and

$$
R^{(n)}=\frac{1+\prod_{i=1}^{n} x_{i}}{1-\prod_{i=1}^{n} x_{i}}
$$

$\S$ The Fermionic term $G^{(n)}$ has several representations [4]. 
with

$$
\begin{aligned}
& x_{i}=\frac{2 w}{1-2 w \cos \left(\phi_{i}\right)+\sqrt{\left(1-2 w \cos \left(\phi_{i}\right)\right)^{2}-4 w^{2}}}, \\
& y_{i}=\frac{2 w}{\sqrt{\left(1-2 w \cos \left(\phi_{i}\right)\right)^{2}-4 w^{2}}}, \quad \sum_{j=1}^{n} \phi_{j}=0
\end{aligned}
$$

valid for small $w$ and, elsewhere, by analytic continuation. The variable $w$ corresponds to small values of $s$ as well as to large values of $s$. In discussing analytic properties of $\tilde{\chi}^{(n)}$ we will often refer to the principal disc by which we mean $|s| \leq 1$ for $\tilde{\chi}^{(2 n+1)}$ and $|s| \geq 1$ for $\tilde{\chi}^{(2 n)}$. For simplicity in writing we will however always refer to the principal disc as $|s| \leq 1$ for the generic $\tilde{\chi}^{(n)}$ and leave it to the reader to understand the correct high/low temperature distinction.

Next, in 1996, Guttmann and Enting [7] gave compelling arguments (though not a proof) that the anisotropic Ising susceptibility was in a different class of functions to that of most functions of exactly solved lattice models. In particular, both the Ising free-energy and magnetisation are known to be holonomic functions (i.e. differentiably finite or D-finite functions), while the susceptibility, they argued, was not. This is an important clue as to why the susceptibility is, mathematically, a much more difficult problem than the free-energy or magnetisation.

In 1999 and 2000, Nickel [3, 4] suggested that the isotropic Ising susceptibility possessed a natural boundary on the unit circle $|s|=1$. While again not providing a rigorous proof, Nickel's arguments were most compelling. Note that functions with a natural boundary cannot be D-finite.

Then in 2001, Orrick, Nickel, Guttmann and Perk [8] presented a polynomial time algorithm for the generation of the coefficients of the series expansion of the susceptibility, in fact in time $\mathrm{O}\left(N^{6}\right)$ for a series of $N$ terms. From an algebraiccombinatorics viewpoint, a polynomial time algorithm is considered a solution. Naturally, a closed form solution is preferable, both for elegance, and so that the analytic structure can be readily extracted. Furthermore, Orrick et al. [8] gave a detailed asymptotic analysis, based on a series expansion of some 323 terms in both high- and low-temperature expansions, and most crucially, short distance correlation functions as series in $\tau=(1 / s-s) / 2$, the temperature deviation from the critical value. Various exponents and amplitude parameters were extracted to unimaginable accuracy, typically 30 or more digits, and these answered most of the outstanding questions as to the structure of the scaling functions.

Orrick et al. [8] also addressed the question of the implications of the unit (complex s-plane) circle as a natural boundary [3, 4]. Isolated singularities can obviously be "seen" and indeed Orrick et al. needed to subtract or otherwise suppress the effect of a number of such singularities, including a contribution from $\tilde{\chi}^{(6)}$, to achieve the accuracy they did in their critical point analysis. They also presented a heuristic argument and calculation to show that the $n \rightarrow \infty$ accumulation of the $\tilde{\chi}^{(n)}$ singularities implied that expansions in $\tau$ would only be asymptotic. Their prediction of the rate at which expansion coefficients diverge was qualitatively confirmed by numerical results on a toy model, subsequently called the diagonal Ising model by Boukraa et al. [9].

In 2004, Zenine, Boukraa, Hassani and Maillard [10] made an important step towards the understanding of the three-particle contribution $\tilde{\chi}^{(3)}$, based on a novel and powerful method for series expansions [11], followed by an inspired computer search 
for the underlying linear ODE generating the series expansion. They obtained the Fuchsian linear ODE for $\tilde{\chi}^{(3)}$. In 2005, the same group [12] found the Fuchsian linear ODE for $\tilde{\chi}^{(4)}$ by similar methods. An important observation coming out of the $\tilde{\chi}^{(3)}$ work was that there were singularities that had not been predicted by Nickel [3, 4] and that the $\tilde{\chi}^{(n)}$ were in fact much more complicated functions than had been imagined. This gave considerable urgency to finding new results for higher order $\tilde{\chi}^{(n)}$.

Since finding the linear ODE for $\tilde{\chi}^{(n)}$ with $n>4$ is clearly a huge task, another strategy to get some kind of handle on the analytic structure of $\tilde{\chi}^{(n)}$ was considered. A set of simplified integrals were introduced $[13,14]$ by making the integrand of $\tilde{\chi}^{(n)}$ simpler and/or by restricting the multiple integral to an integral over a single variable. The hope was that these integrals will preserve some (or hopefully all) of the singularity structure of the full problem. From these model integral investigations, a reasonably consistent picture of the singularities emerged and a large set of candidate singularities for those actually occurring in the linear ODE's of $\tilde{\chi}^{(n)}$ was obtained.

In this paper we return to the problem of finding linear ODE's for $\tilde{\chi}^{(n)}$ (for $n>4$ ) or, failing that, to obtaining long series that can be analysed by other means. To save space and repetition, we will drop the important adjective linear before ODE in the following, but all mention of ODEs should be taken as a reference to a linear Fuchsian $O D E$, unless otherwise stated. A strong motivation for obtaining such long series for the full susceptibility $\tilde{\chi}$, and the $n$-particle contributions $\tilde{\chi}^{(5)}$ and $\tilde{\chi}^{(6)}$ is not to improve already known numerical calculations (critical exponents, amplitudes, ...), but to shed some light on important, and not yet understood, physical problems such as the structure of the ODE's they satisfy, the implication of new singularities for the natural boundary, heuristic asymptotics of Orrick et al. [8], and the mechanism of resummation of logarithmic singularities.

Indeed, in all previous analyses of the full $\chi$ and the individual $\chi^{(n)}$, a point appeared that was left unresolved [15]. This issue is the power/log behaviour of each $\chi^{(n)}$ at the singular points which is not the same as the behaviour of the full $\chi$. That is to say, the asymptotic behaviour of the full susceptibility $\chi$ is (with $\tau=\frac{1}{2}(1 / s-s)$ )

$$
\chi \sim \text { ct. }|\tau|^{-7 / 4}+\text { ct. }+ \text { ct. }|\tau|^{1 / 4}+\mathrm{O}(|\tau| \log |\tau|),
$$

whereas:

$$
\chi^{(n)} \sim c t .|\tau|^{-7 / 4}+c t .|\tau|^{1 / 4}(\log |\tau|)^{n-1}+\mathrm{O}\left(\log |\tau|^{n-2}\right),
$$

where $c t$. denotes constants. In order to resolve the issue of how the individual terms of the form $|\tau|^{1 / 4}(\log |\tau|)^{n-1}$ occurring in $\chi^{(n)}$ combine to give a constant in the full susceptibility, we require a better understanding of each $\chi^{(n)}$ rather than getting the full $\chi$ to higher accuracy. With the complete solutions for $\tilde{\chi}^{(3)}$ and $\tilde{\chi}^{(4)}$, long series expansions for the higher terms $\chi^{(n)}$ and for the full $\chi$ will allow us to completely resolve this issue.

Our achievements, with regard to series generation, are that we have extended the series for the full $\chi, \tilde{\chi}^{(5)}$ and $\tilde{\chi}^{(6)}$ to about 2000 termsई. We also have series for $\tilde{\chi}^{(5)}$, modulo a single prime, to 10000 terms, and this has enabled us to find the associated linear ODE modulo a prime and get a complete picture of the singularities and exponents in that case. Most of the details of this $\tilde{\chi}^{(5)}$ linear ODE analysis will be given in a future paper.

In this paper we report in Section 2 on how the generation of the long series has been achieved and in Section 3 we describe an efficient method for finding the

$\S$ More precisely, for $\tilde{\chi}^{(6)}$ we have 3260 coefficients in $w$ or 1616 non-zero terms (that is in $x=w^{2}$ ). 
associated linear ODE's and give some results from the linear ODE mod prime analysis of $\tilde{\chi}^{(5)}$. Section 4 is devoted to a floating-point diff-Padé analysis of the 2000 terms exact series for $\tilde{\chi}^{(5)}$. There is no such numerical work on long series in the literature and so we have had no a priori knowledge about what to expect. In view of this, we have been strongly motivated to make a detailed comparison between the numerical and exact $\tilde{\chi}^{(5)}$ results. The comparison has been instructive and we now have a reasonably good basis for judging the efficacy and reliability of the diff-Padé method. Specifically, there are clear patterns showing which singularities and exponents can be obtained and which will be missed. We report also on the floating-point diff-Padé analysis of the 1600 term exact series for $\tilde{\chi}^{(6)}$ where only limited exact results are known based on our previous $[13,14]$ toy model investigations. Section 5 is devoted to a study of the singularities that occur in the higher $\tilde{\chi}^{(n)}, n \geq 7$, by performing a diff-Padé analysis on the full $\tilde{\chi}$ series from which the small $n$ contributions $\tilde{\chi}^{(n)}$, $n<7$ terms have been removed. We address in Section 6 the "logarithm summation" problem discussed above, and resolve it. A simplified model is introduced to show how the subdominant singularities in $\chi^{(n)}$, that individually vanish as the temperature approaches its critical value, sum to yield the known finite amplitude at the critical point. In Section 7 we describe the fast Fourier transform (FFT) that was used by Orrick et al. [8] as a diagnostic tool in the study of series singularities. Here we use it to confirm the absence of certain singularities on the complex $s$-plane unit circle and confirm the existence of a natural boundary for $\chi$.

Except for Appendix A on Fuchs' relations, the Appendices revisit and extend the Landau singularity analysis $[13,14,16]$ of $\tilde{\chi}^{(n)}$. In Appendix B we prove that in the absence of the "Fermionic factor" $(G(n))^{2}$ in (5), the singularities found in $[13,14]$ are exhaustive. We also prove that none of these singularities, beyond those found by Nickel [3], can lie on the principal $s$-plane unit circle. This dispels any hope that there might be singularity cancellation and that, as a result, the unit circle may not be a natural boundary.

The presence of the Fermionic factor in the $\tilde{\chi}^{(n)}$ integral (5) is too complicated to deal with in its entirety, and Appendices B and D provide a more limited perspective. We describe in Appendix D what happens when all, but a single term of the complete Fermionic product, is dropped from the integrand of $\tilde{\chi}^{(n)}$. The linear ODE describing the series in that case has additional singularities. On the other hand we show that there are no such Landau singularities $[13,14]$ on various analytic continuations $\$$ of the $\tilde{\chi}^{(n)}$. The conclusion seems to be that the singularities of the integral representation of $\tilde{\chi}^{(n)}$ do not identify with but are just included into singularities of the linear ODE associated with a $\tilde{\chi}^{(n)}$. In Appendix $\mathrm{E}$ we give a power counting argument to determine the singularity exponents of one class of Landau singularities. The close analogy of these to the principal $s$-plane unit circle singularities, and the simple formula for the exponent values, motivate us to call them the "normal" exponents. Other exponents are known to be present based on the exact and diff-Padé analysis in Sections 4 and 5 , and, as a tentative terminology, we denote these other exponents as "anomalous".

9 The Landau singularity analysis $[13,14]$ is local. This amounts to keeping no track of which local square-root branch we are on for each singularity. 


\section{Extremely long series for the susceptibility}

\subsection{The full susceptibility $\chi$}

Orrick et al. [8] used an algorithm of complexity $\mathrm{O}\left(N^{6}\right)$ to obtain the first $N$ coefficients of the susceptibility series. As a result, they generated and analysed series with more than 300 terms in both the high- and low-temperature regime. This remarkable polynomial growth of the algorithm is due to the use of quadratic partial difference equations (see [8]) which are finite difference Painlevé equations [17, 18].

The susceptibility $\chi$ is obtained via (2) with this quadratic partial difference system of equations providing a means to calculate the two-point correlations $C(m, n)$ efficiently, and simultaneously, for both high- and low-temperature series. A susceptibility series of $N$ terms requires $C(m, n)$ on the octant $m+n \leq 2 N$, $m<n$, deduced as series from the quadratic difference equations, with the diagonal $C(n, n), n \leq N$, as initial value data. The latter can be obtained from either a Toeplitz determinant expression [17] or a set of non-linear recursion relations [19]. The calculation of $C(m, n)$ on a particular site requires the multiplication of the $C\left(m^{\prime}, n^{\prime}\right)$ series of length $\mathrm{O}(N)$ for the same and/or neighbouring sites. If the series multiplication is done as a naive product then the complexity of the susceptibility determination is that of $\mathrm{O}\left(N^{2}\right)$ integer multiplications on $\mathrm{O}\left(N^{2}\right)$ sites. The Maple code used by Orrick et al. [8] ran in a time approximately proportional to $N^{6}$. This was as expected since the integer (digit) size grows linearly in $N$ and naive integer multiplication scales as $N^{2}$. There was no attempt to improve on this code as the 323 term series obtained was deemed entirely adequate.

Obvious improvements can be made. First, we know that $\chi$ when expressed in the variable $s / 2$ (high temperature) or $1 /\left(4 s^{2}\right)$ (low temperature) is a series with integer coefficients. This implies that if the series generating program is run using modular arithmetic [20] with a number of different primes, then the full series coefficients can be reconstructed from the residue series via the Chinese remainder theorem. The number of primes necessary is easily estimated. Since $|s|=1$ is the singularity boundary for both high and low temperatures, the high-temperature series coefficients in $s / 2$ grow with the number of terms $N$ as $2^{N}$, while at low temperature the series coefficients in $1 /\left(4 s^{2}\right)$ grow as $4^{N}$. The latter expressed in numbers of bits is $2 N$ and with primes of bit length 15 that we typically use, we arrive at $\simeq 2 N / 15$ as the required number of primes.

The modular arithmetic approach leaves the complexity of the $\chi$ series generation to $N$ terms at $\mathrm{O}\left(N^{4}\right)$ multiplications for a single prime. For the full calculation that requires $\mathrm{O}(N)$ primes the complexity is $\mathrm{O}\left(N^{5}\right)$. However, once the generating program is running in integer mod prime mode, it is easy to implement series multiplication via fast Fourier transform (FFT) [20]. This changes the series multiplications from $\mathrm{O}\left(N^{2}\right)$ to $\mathrm{O}(N \log (N))$ and gives as our final estimate for the $\chi$ generation complexity $\mathrm{O}\left(N^{4} \log (N)\right)$. In the next subsection we show how similar complexity improvements in the generation of the $\tilde{\chi}^{(n)}$ series have been achieved.

For the extension of the $\chi$ series to 2000 terms as reported below, we translated the Maple code from Orrick et al. [8] to Fortran 9 and ran it in integer mod prime mode. We also incorporated the FFT multiplication of series. Finally we changed the $C(n, n)$ initialization to use the Jimbo and Miwa recursion [19].

I In preliminary studies we obtained 1600 terms of the high and low temperature expansions of $\chi$ with highly optimised $\mathrm{C}++$ programs. 
With these changes the calculation on the APAC (Australian Partnership for Advanced Computing) SGI Altrix cluster with $1.6 \mathrm{GHz}$ Itanium2 processors using 280 primes took about $240 \mathrm{CPU}$ hours in total (we note that the algorithm without FFT multiplication would require about twice the above amount of CPU time).

The resulting series can be found on the web-page [21] where we give the first 2000 coefficients of the high- and low-temperature expansions in the variables $u=s / 2$ and $v=1 /\left(4 s^{2}\right)$, respectively. In addition we also give the expansion for $\tilde{\chi}_{H}$ and $\tilde{\chi}_{L}$ in the self-dual variable $w=\frac{1}{2} s /\left(1+s^{2}\right)$. The series in the $u$ or $v$ variables, or in the $w$ variable, are probably the best as far as computer encoding and modular calculations are concerned since all the coefficients are integers rather than rational numbers. For example, for any $n>2, \tilde{\chi}^{(n)}=2^{n} w^{n^{2}}\left[1+4 n^{2} w^{2}+\mathrm{O}\left(w^{3}\right)\right]$.

\subsection{The contributions $\tilde{\chi}^{(5)}$ and $\tilde{\chi}^{(6)}$}

The previous longest series available for $\chi^{(5)}$ and $\chi^{(6)}$ can be found in [8] where the first 182 and 140 terms respectively of the series in $s$ are listed. We have extended these series dramatically - two extreme examples being the full integer series for $\tilde{\chi}^{(5)}$ to 2000 terms and the series for $\tilde{\chi}^{(5)}$ modulo a single prime to 10000 terms. The latter extension is only possible because the complexity order for a mod prime series of length $N$ has been reduced to $\mathrm{O}\left(N^{4} \log (N)\right)$. We outline in this section how this reduction has been achieved.

Our method for evaluating the integral expression (5) for $\tilde{\chi}^{(n)}$ remains as described in earlier publications [11, 12]. We first convert (5) back to an $n$-fold $\phi_{i}$ integration with the explicit phase constraint $2 \pi \delta\left(\sum \phi_{i}\right)$ now in the integrand. This constraint is then replaced by the equivalent $\sum_{k} \exp \left(\mathrm{i} k \sum \phi_{i}\right)$, thus decoupling all $\phi_{i}$ integrations at the expense of a sum over the Fourier integer $k$, where the sum extends from $k=-\infty$ to $\infty$.

Next we expand all denominator factors in the integrand of $\tilde{\chi}^{(n)}$, thereby converting the integrand into a sum of $n$-fold products $\prod y_{i} x_{i}^{n_{i}}$. Each $\phi_{i}$ integration then picks out the $k^{t h}$ Fourier coefficient of $y_{i} x_{i}^{n_{i}}$, namely $w^{|k|+n_{i}+1} \cdot a\left(k, n_{i}\right)$, where $a\left(k, n_{i}\right)$ is proportional to a hypergeometric function ${ }_{4} F_{3}$ in the variable $16 w^{2}$. The net result of all these operations is that we have replaced the $\tilde{\chi}^{(n)}$ continuum integration by a nested summation of products of hypergeometric functions.

The complexity of this calculation is of some order that we can now easily determine. If we want a series of length $N$, then the Fourier $k$ sum can be restricted to $\mathrm{O}(N)$ as can all the hypergeometric function series. The evaluation of products of series of length $\mathrm{O}(N)$ is either an $\mathrm{O}\left(N^{2}\right)$ calculation if done as a naive product or $\mathrm{O}(N \log (N))$ if done by FFT. These two operations, namely the $k$ sum and the series multiplication, are inherent to our method and give an irreducible minimum complexity of either $O\left(N^{3}\right)$ or $\mathrm{O}\left(N^{2} \log (N)\right)$. The only place where we have some freedom to reduce the complexity of our $\tilde{\chi}^{(n)}$ series evaluation is in the number of summations that are required for the expansion of the denominator factors in the original $\tilde{\chi}^{(n)}$ integrand. The total number of products $\prod y_{i} x_{i}^{n_{i}}$ must be no more than $\mathrm{O}\left(N^{2}\right)$ to keep the overall complexity at $\mathrm{O}\left(N^{4} \log (N)\right)$ and this, in turn, implies the denominator expansion must be limited to two independent summations.

I Trigonometric functions in the integrand such as $\sin \left(\left(\phi_{i}-\phi_{j}\right) / 2\right)$ are dealt with by expanding them into a sum of phases $\exp \left(i \sum m_{i} \phi_{i}\right)$ with each $m_{i}$ some small integer. These can be incorporated into shifts $k \phi_{i} \rightarrow\left(k+m_{i}\right) \cdot \phi_{i}$ and do not change the form of the integrand. Since such shifts also do not change the complexity order of the $\tilde{\chi}^{(n)}$ calculation they will not be considered further. 
This limitation on the summations immediately shows that the product form (6) for the Fermionic factor $G^{(n)}$ is not appropriate and alternatives must be used. The useful formulae for high-temperature series are those given in [3], in particular equation (5) for some low order $\tilde{\chi}^{(2 n+1)}$ and equation (10) for the general case. For low-temperature series we note that $G^{(2 n)}$ has been shown to be a Pfaffian [22] in the $h_{i j}$ defined in (6). By a rearrangement of terms in the Pfaffian one finds that the analog of equation (10) in [3] is

$$
\begin{aligned}
& H^{(2 n)}=\frac{\left(G^{(2 n)}\right)^{2}}{(2 n) !}=\frac{1}{(2 n)} h_{12} h_{2 n-1,2 n} \\
& \times \prod_{m=1}^{n-1}\left(h_{2 m, 2 m+1} h_{2 m-1,2 m+2}+\frac{1}{(2 m)} h_{2 m-1,2 m} h_{2 m+1,2 m+2}\right) .
\end{aligned}
$$

Use of label interchange symmetry, $h_{i j}=-h_{j i}$, allows one to combine terms in (12) further. For the first few low order terms we have

$$
\begin{aligned}
H^{(2)} & =-\frac{1}{2}\left(h_{12} h_{21}\right), \\
H^{(4)} & =\frac{1}{8}\left(h_{12} h_{21}\right)\left(h_{34} h_{43}\right)-\frac{1}{4}\left(h_{12} h_{23} h_{34} h_{41}\right), \\
H^{(6)} & =-\frac{1}{48}\left(h_{12} h_{21}\right)\left(h_{34} h_{43}\right)\left(h_{56} h_{65}\right)+\frac{1}{8}\left(h_{12} h_{23} h_{34} h_{41}\right)\left(h_{56} h_{65}\right) \\
& -\frac{1}{6}\left(h_{12} h_{23} h_{34} h_{45} h_{56} h_{61}\right),
\end{aligned}
$$

written in an obvious cyclic form. It is these expressions, in particular the one for $H^{(6)}$, that are the starting point of our discussion of the reduction in the denominator expansion summations. The most complicated term contributing to $\tilde{\chi}^{(6)}$ has an integrand that contains the last term of $H^{(6)}$ in (13) and thus the seven denominator factors

$$
\begin{aligned}
& \left(1-x_{1} x_{2} x_{3} x_{4} x_{5} x_{6}\right)^{-1}\left(1-x_{1} x_{2}\right)^{-1}\left(1-x_{2} x_{3}\right)^{-1} \\
& \times\left(1-x_{3} x_{4}\right)^{-1}\left(1-x_{4} x_{5}\right)^{-1}\left(1-x_{5} x_{6}\right)^{-1}\left(1-x_{6} x_{1}\right)^{-1} .
\end{aligned}
$$

Naive expansion of these denominators results in a 7 -fold sum - clearly a 5 -fold excess that must be eliminated. The first step towards this elimination is the use of partial fraction rearrangement, a trick that was already used in the evaluation of $\tilde{\chi}^{(3)}$ and $\tilde{\chi}^{(4)}$ in $[11,12]$. The version we use here is based on the identity

$$
\begin{aligned}
& (1-A)^{-1}(1-B)^{-1}(1-C)^{-1}= \\
& \quad(1-A B C)^{-1}\left[1-(1-A)^{-1}-(1-B)^{-1}-(1-C)^{-1}+\right. \\
& \left.\quad(1-A)^{-1}(1-B)^{-1}+(1-B)^{-1}(1-C)^{-1}+(1-C)^{-1}(1-A)^{-1}\right],
\end{aligned}
$$

in which we first set $A, B, C=x_{1} x_{2}, x_{3} x_{4}, x_{5} x_{6}$ and then $A, B, C=$ $x_{2} x_{3}, x_{4} x_{5}, x_{6} x_{1}$. The product of these two forms of (15) enables us to replace (14) by

$$
\begin{gathered}
\left(1-x_{1} x_{2} x_{3} x_{4} x_{5} x_{6}\right)^{-3}\left(1-x_{1} x_{2}\right)^{-1}\left(1-x_{2} x_{3}\right)^{-1} \\
\times\left(1-x_{3} x_{4}\right)^{-1}\left(1-x_{4} x_{5}\right)^{-1}
\end{gathered}
$$

plus terms with similar, or fewer, denominators. That is, (16) is now the most complicated set of denominators in the integrand contributing to $\tilde{\chi}^{(6)}$. With the replacement of the first factor by $\left(1-x_{1} x_{2} x_{3} x_{4} x_{5}\right)^{-1}$, (16) becomes the equivalent 
most complicated term in the evaluation of $\tilde{\chi}^{(5)}$. Thus all our subsequent remarks apply equally to both $\tilde{\chi}^{(5)}$ and $\tilde{\chi}^{(6)}$.

Expansion of (16) results in the formal 5-fold summation

$$
S=\sum_{m, n_{1}, p, q, n_{5}} x_{1}^{m+n_{1}} x_{2}^{m+n_{1}+p} x_{3}^{m+p+q} x_{4}^{m+q+n_{5}} x_{5}^{m+n_{5}} x_{6}^{m},
$$

which is of complexity $\mathrm{O}\left(N^{5}\right)$. However the $n_{1}$ and $n_{5}$ summations can be done independently and thus (17) is in fact only of complexity $\mathrm{O}\left(N^{3} \cdot(N+N)\right)=\mathrm{O}\left(N^{4}\right)$. This is not yet an adequate reduction and we can do better by eliminating the $n_{1}$ and $n_{5}$ summations entirely via the use of recursion relations. For example, we define the $n_{1}$ sum of the $x_{1}, x_{2}$ pair as

$$
A_{12}(m, p)=\sum_{n_{1}=0} x_{1}^{m+n_{1}} x_{2}^{m+n_{1}+p}=\sum_{r=m} x_{1}^{r} x_{2}^{r+p}
$$

and note that because $m$ appears only as a limit on a dummy variable sum, the $A_{12}$ satisfies the recursion

$$
\begin{aligned}
& A_{12}(m, p)=\delta A_{12}(m, p)+A_{12}(m+1, p), \\
& \delta A_{12}(m, p)=x_{1}^{m} x_{2}^{m+p} .
\end{aligned}
$$

Only the lower limit on the summation in (18) has been given explicitly. There is also an upper limit that depends on the length $N$ of the series in $\tilde{\chi}^{(5)}$ or $\tilde{\chi}^{(6)}$ we want to obtain. Thus $A_{12}(m, p)$ vanishes for $m$ large enough, and (19) shows that, as $m$ is decreased from this upper limit, each determination of $A_{12}(m, p)$ requires only the evaluation of a single product and its accumulation into a previously stored result $\ddagger$. If we now take it as given that the $m$ summation in (17) is performed in decreasing sequence we obtain

$$
S=\sum_{m, p, q} A_{12}(m, p) \cdot x_{3}^{m+p+q} \cdot A_{54}(m, q) \cdot x_{6}^{m},
$$

which is of complexity $\mathrm{O}\left(N^{3}\right)$.

It remains to be shown that the idea of recursion can be applied once more, ultimately reducing the complexity of the calculation of $S$ to $\mathrm{O}\left(N^{2}\right)$. For this purpose define

$$
B_{123}(m, q)=\sum_{p=0} A_{12}(m, p) x_{3}^{m+p+q},
$$

which, for $q>0$, can be put in the recursive form

$$
\begin{aligned}
B_{123}(m, q) & =\sum_{p=0}\left[\delta A_{12}(m, p)+A_{12}(m+1, p)\right] x_{3}^{m+p+q} \\
& =\delta B_{123}(m, q)+B_{123}(m+1, q-1) .
\end{aligned}
$$

For $\delta B_{123}(m, q)$, which is the first sum in (22), we have

$$
\delta B_{123}(m, q)=\sum_{p=0} x_{1}^{m} x_{2}^{m+p} x_{3}^{m+p+q}=x_{1}^{m} \cdot A_{23}(m, q),
$$

$\ddagger$ Our discussion here is schematic. It is to be understood that the $\phi_{i}$ integrations have been carried out and the "single product" being referred to is the product of the two hypergeometric function series associated with $x_{1}^{m} x_{2}^{m+p}$. An additional implication is that $A_{12}(m, p)$ has absolutely no functional dependence on its subscripts and could equally well be denoted $A(m, p)$. The subscripts have only been included to indicate a connection to a particular factor in the $\tilde{\chi}^{(5)}$ or $\tilde{\chi}^{(6)}$ integrand. 
a single product analogous to $\delta A_{12}$ in (19). The new feature in the recursion (22) for $B_{123}$, relative to (19) for $A_{12}$, is that we must maintain in storage an entire array of elements indexed by $q$. Furthermore, for each $m$ we must supply, by a separate calculation, the $q=0$ term

$$
B_{123}(m, 0)=\sum_{p=0} A_{12}(m, p) \cdot x_{3}^{m+p} .
$$

Although this does require a sum, the fact that $q=0$ is fixed means the contribution of this evaluation to the complexity order of $S$ is still only $\mathrm{O}\left(N^{2}\right)$. With the general $B_{123}$ now given either by (22) or (24), we obtain

$$
S=\sum_{m, q} B_{123}(m, q) \cdot A_{54}(m, q) \cdot x_{6}^{m},
$$

which is the two-fold sum, and thus the $\mathrm{O}\left(N^{2}\right)$ result, we were looking for. Note that memory requirements are also quite minimal. We need to store the $B$ array which has $\mathrm{O}(N)$ elements each of which is a series of length $\mathrm{O}(N)$. Thus memory requirements also scale as $N^{2}$.

Our initial Fortran coding for the $\tilde{\chi}^{(5)}$ and $\tilde{\chi}^{(6)}$ series generation used only naive series multiplication and thus was $O\left(N^{5}\right)$ for a single prime. With these programs, series for $\tilde{\chi}^{(5)}$ to 2000 terms and $\tilde{\chi}^{(6)}$ to 3260 terms were generated in about 100000 CPU hours running 160 primes in parallel. The calculations were carried out on the afore-mentioned APAC cluster. Series for $\tilde{\chi}^{(5)}$ to 6000 terms, modulo the single prime $p_{0}=32749$, were obtained in about $40000 \mathrm{CPU}$ hours using 32 processors on one of the VPAC (Victorian Partnership for Advanced Computing) facilities which is a Linux cluster based on Xeon 2.8Ghz CPUs.

When it was observed that the 6000 terms were not sufficient to obtain the linear ODE for $\tilde{\chi}^{(5)}$, the Fortran codes were modified to include FFT series multiplication and series modulo $p_{0}$ for $\tilde{\chi}^{(5)}$ to 10000 terms were obtained.

The total CPU time for $\tilde{\chi}^{(5)}$ to 10000 terms was about 17000 hours (6000 terms take around 2000 hours using the FFT algorithm on the APAC).

The calculation was done in parallel using 128 processors. The algorithm is straightforward to parallelise because the calculations in the outer most loop, that is the sum over Fourier mode integer $k$, can be done independently for each value of $k$. The only issue is that the time required decreases with $k$. In order to use approximately the same time per processor we simply assign calculations with a given $k$ to processors in an alternating pattern such that processor 0 does $k=0, k=255$, $k=256, k=512, k=513, \ldots$ while processor 1 does $k=1, k=254, k=257$, $k=511, k=514, \ldots$ and so on up to processor 127 which does $k=127, k=128$, $k=383, k=384, k=639, \ldots$. This simple assignment scheme ensures a good balance with the total time used by various processors differing by less than $4 \%$.

It is this 10000 terms series that has enabled us to obtain the exact ODE modulo $p_{0}$ for $\tilde{\chi}^{(5)}$, thus making possible the various comparisons found elsewhere in this paper.

We conclude with two observations on the technical aspects of the mod prime calculations. Firstly, a very frequent operation in our codes is the accumulation of two products, i.e. $a \rightarrow a+b c+d e$. If the variables are integers modulo $p$ then the accumulation can be done as a standard 32 bit integer operation with the single (Fortran) call $a=\bmod (a+b c+d e, p)$ without overflow provided $p<2^{15}$. It is this feature that dictates our choice of primes and in particular $p_{0}=2^{15}-19$. Secondly, 
we follow a recommended practice [23] of loading the floating point FFT routines with mod prime variables in "balanced" form. That is, if any mod $p$ variable $v$ is greater than $p / 2$ it is loaded as $v-p$. This eliminates most of the "dc" part of the input signal and typically increases the safety margin in the output rounding of float to integer by several bits. We keep track of the differences in these rounding operations so as to guarantee our programs generate all integers correctly.

\section{Fuchsian ODEs for long series modulo a prime}

\subsection{The linear ODE for $\tilde{\chi}^{(5)}$}

We begin with a remark that applies to all the subsequent discussion, namely that there is no single unique $O D E$ that describes any given series. There is a minimum order linear ODE that is unique but this typically contains a very large number of apparent singularities and can only be determined from a corresponding larger number of series coefficients. In our quest for linear ODE's corresponding to given very long series expansions, we are interested in the Fuchsian linear ODE requiring the minimum number of coefficients from the series in order to be obtained. In general there are any number of intermediate ODE's but unless otherwise required by the context, we will call all of these the underlying linear ODE, without distinction.

The 2000 terms generated for $\tilde{\chi}^{(5)}$ and $\tilde{\chi}^{(6)}$ are not sufficient to find the exact underlying linear ODE. However an alternative approach is to use mod prime series to find the linear ODE modulo a prime. From such a linear ODE we can get singularity positions modulo a prime, indicial equations and hence singularity exponents modulo a prime, and indeed practically everything that could be obtained from the exact ODE but restricted to prime residues. Furthermore if, for example, the modulo prime factorization of the head polynomial of the linear ODE yields factors with small coefficients, then one can, with almost perfect certainty, conclude that one has all the exact singularity locations. Similar remarks would also apply, say, to singularity exponentsł. And indeed, because we have been able to find the mod prime linear ODE for $\tilde{\chi}^{(5)}$ and because the singularity locations and exponents appear to be simple, we are confident that what we report are in fact the exact values.

Because series generation is expensive we want to be sure our algorithm for deducing the underlying ODE requires the fewest number of terms. We report in this section on a method [24] that is slightly different from that used for, say, $\tilde{\chi}^{(3)}$ but that appears to have a number of appealing advantages. We will report on some of our results on the analysis of $\tilde{\chi}^{(5)}$, in particular those that affect the number of series terms required to find the ODE. Most of the details, such as the factorization of the linear ODE, will be left for a future publication.

An essential constraint on the linear ODE underlying a series $S(x)$ of the type we are considering here is that it must be Fuchsian. Specifically this means that $x=0$ and $x=\infty$ are regular singular points. A form for the linear differential operator that automatically satisfies this $(0, \infty)$ regularity constraint is:

$$
L_{M D}=\sum_{m=0}^{M} \sum_{d=0}^{D} a_{m d} \cdot x^{d} \cdot\left(x \frac{\mathrm{d}}{\mathrm{d} x}\right)^{m}, \quad a_{M 0} \neq 0, a_{M D} \neq 0 .
$$

$\ddagger$ Of course, if there is an ambiguity one can always use additional primes and resolve the ambiguity by the Chinese remainder theorem. 
The $a_{M 0} \neq 0$ condition is the obvious constraint to make $x=0$ a regular singular point and it is the use of the operator $x \frac{\mathrm{d}}{\mathrm{d} x}$ rather than $\mathrm{d} / \mathrm{d} x$ that makes analysis around $x=\infty$ simple. The change of variable $x=1 / y$ turns (26) into

$$
L_{M D}=\sum_{m=0}^{M} \sum_{d=0}^{D} a_{m d} \cdot y^{D-d} \cdot\left(-y \frac{\mathrm{d}}{\mathrm{d} y}\right)^{m}, \quad a_{M 0} \neq 0, a_{M D} \neq 0,
$$

where one can see that the condition for $x=\infty(y=0)$ to be a regular singular point is $a_{M D} \neq 0$. A simple rearrangement of terms shows that $L_{M D}$ can also be written

$$
L_{M D}=\sum_{m=0}^{M} \sum_{d=0}^{D} b_{m d} \cdot x^{d+m} \cdot\left(\frac{\mathrm{d}}{\mathrm{d} x}\right)^{m},
$$

with the $b$ coefficients being linear combinations of the $a$. There is no single $b$ coefficient analog of $a_{M D} \neq 0$. We will use (26) exclusively, particularly for the purpose of determining the $a_{m d}$, but this does not preclude transforming to (28) if required.

To determine the $a_{m d}$ in (26) we demand $L_{M D}(S(x))=0$ and this yields a set of linear equations that we arrange in some well defined order. There exists a nontrivial solution if the $N_{M D} \times N_{M D}$ determinant (with $N_{M D}=(M+1) \cdot(D+1)$ ) corresponding to the chosen ordering vanishes. We test this by standard Gaussian elimination, creating an upper triangular matrix $U$ in the process. If we find $U(N, N)=0$ for some $N$, a non-trivial solution exists. If $N<N_{M D}$ we set to zero all $a_{m d}$ in the ordered list beyond $N$. Of the remaining $a_{m d}$ we set $a_{M 0}=1$, thus guaranteeing that $x=0$ is a regular singular point, and determine the rest by back substitution. We guarantee that $x=\infty$ is a regular singular point by choosing the initial ordering of elements such that no matter what $N$ is, the first elements set to zero will be those from row $a_{0 d}$, then row $a_{1 d}$, etc. In this way the element $a_{M D}$ will never vanish unless all $a_{m D}, m<M$ vanish or the series $S(x)$ has $x=\infty$ as an irregular singular point. We have not systematically investigated what happens when we change the element ordering within these constraints.

The $N$ for which $U(N, N)=0$ is the minimum number of coefficients needed to find the linear ODE within the constraint of a given $M$ and $D$. Obviously, $N \leq N_{M D}=(M+1) \cdot(D+1)$. Henceforth, $D$ will always refer to the minimum $D$ for which a solution is found for the given $M$. Then we can define a unique nonnegative deviation $\Delta$ by $N=N_{M D}-\Delta$. Examples of such constants are given in Table 1 based on our analysis of $\tilde{\chi}^{(5)}$ and the combination $2 \cdot \tilde{\chi}^{(5)}-\tilde{\chi}^{(3)}$.

A very striking empirical observation arises from Table 1 and has been checked in many cases as summarised in Table 2. For reasons we do not understand, there exists the linear relationship

$$
N=A \cdot M+B \cdot D-C=(M+1) \cdot(D+1)-\Delta
$$

where $A, B$ and $C$ are constants depending on the particular series $S(x)$. For $\tilde{\chi}^{(5)}$ they are $A=72, B=33, C=900$, while for the combination $2 \cdot \tilde{\chi}^{(5)}-\tilde{\chi}^{(3)}$ they are $A=68, B=30, C=744$ as can be verified from Table 1 . Note that (29) has no (positive) solution for $D$ if $M<B$. Thus $B=M_{0}$ is the minimum order possible for the linear differential operator that annihilates $S(x) \S$. Similarly, $A=D_{0}$ is the minimum possible degree and thus we can rewrite (29) in the more definitive form

$$
N=D_{0} \cdot M+M_{0} \cdot D-C=(M+1) \cdot(D+1)-\Delta .
$$

$\S$ Generically $B=M_{0}$. However, if the ODE is such that $a_{0 d}=0$ for all $d$ then the constant $B=M_{0}-1$, that is, the minimum order minus 1 . 
Table 1. $M$ is the order of the linear ODE, $D$ is the degree of each polynomial multiplying each derivative, $N_{M D}=(M+1)(D+1), N$ is the actual number of terms predicted by (29) as necessary to find an ODE of the given order $M$, and $\Delta$ is the difference $N_{M D}-N$. The first five columns gives this data for $\tilde{\chi}^{(5)}$ while the next five columns gives this data for $2 \cdot \tilde{\chi}^{(5)}-\tilde{\chi}^{(3)}$, clearly showing the saving in the number of terms needed to identify the linear ODE.

\begin{tabular}{|c|c|c|c|c||c|c|c|c|c|}
\hline \multicolumn{9}{|c|}{ Terms needed to find $\tilde{\chi}^{(5)}$ Terms needed to find $2 \cdot \tilde{\chi}^{(5)}-\tilde{\chi}^{(3)}$} \\
\hline$M$ & $D$ & $N_{M D}$ & $N$ & $\Delta$ & $M$ & $D$ & $N_{M D}$ & $N$ & $\Delta$ \\
\hline 52 & 141 & 7526 & 7497 & 29 & 48 & 131 & 6468 & 6450 & 18 \\
53 & 137 & 7452 & 7437 & 15 & 49 & 128 & 6450 & 6428 & 22 \\
54 & 134 & 7425 & 7410 & 15 & 50 & 125 & 6426 & 6406 & 20 \\
55 & 132 & 7448 & 7416 & 32 & 51 & 123 & 6448 & 6414 & 34 \\
56 & 129 & 7410 & 7389 & 21 & 52 & 120 & 6413 & 6392 & 21 \\
57 & 127 & 7424 & 7395 & 29 & 53 & 118 & 6426 & 6400 & 26 \\
58 & 125 & 7434 & 7401 & 33 & 54 & 116 & 6435 & 6408 & 27 \\
59 & 123 & 7440 & 7407 & 33 & 55 & 114 & 6440 & 6416 & 24 \\
60 & 121 & 7442 & 7413 & 29 & 56 & 112 & 6441 & 6424 & 17 \\
61 & 119 & 7440 & 7419 & 21 & 57 & 111 & 6496 & 6462 & 34 \\
\hline
\end{tabular}

The minimum order $M_{0}$ and degree $D_{0}$ can also be inferred directly from the ODE independently of (30).

The head polynomial $\sum_{d=0}^{D} a_{m d} x^{d}$ in (26) can be factored modulo a prime and the greatest common divisor of these, from several different $L_{M D}$, is the polynomial $P$ whose zeros are the "true singularities" of the linear ODE. In all cases we have tested, the degree of this head polynomial factor is the $D_{0}$ in $(30)$. For $\tilde{\chi}^{(5)}(w)$ the factor, $P(w)=P_{D_{0}}(w)=P_{72}(w)$, is sufficiently simple that we are confident that it equals what one would obtain from the exact (not modulo a prime) ODE. We report it (cf. (34)) in Section 3.3 in the context of a more general discussion of singularities and make extensive use of it in Section 4 for comparison purposes with results obtained from floating-point diff-Padé analysis of the 2000 term exact $\tilde{\chi}^{(5)}$ series.

The multiplicity of any zero $x=x_{s}$ of $P_{D_{0}}(x)$ is the number of linearly independent singular functions in the neighbourhood of $x_{s} \sharp$. If we add to this list of multiplicities the number of independent singular functions at $x=0$ and at $y=1 / x=0$, then the maximum multiplicity is the minimum order of the ODE. We have again verified that this agrees with $M_{0}$ in (30), although there is a subtlety to determining the true multiplicities at $x=0$ and $y=0$. By our definition of $L_{M D}$ in (26) the indicial equations at these points are, $\sum_{m=0}^{M} a_{m 0} \cdot p^{m}=0$, and $\sum_{m=0}^{M} a_{m D} \cdot(-p)^{m}=0$, respectively, and thus of degree $M$. If there are positive integer roots then, even if we factor these indicial equations modulo a prime for several $L_{M D}$ and take the greatest common divisor, we can only be sure that the true multiplicity is less than or equal to the degree of the greatest common divisor. The ambiguity at $x=\infty$ is easily resolved by analysing the transformed series $S(z)$ where $x=z /(1-z / \alpha)$, thus mapping $x=\infty$ to $z=\alpha$. In all our examples the situation at $x=0$ has been unambiguous but we do not see why this would be the

$\sharp$ There is no difficulty in finding the roots of the associated indicial equation mod prime. In the case of $\tilde{\chi}^{(5)}$ we are again confident that we have all the exact exponents and these are reported in Section 4 . 
case in general.

To interpret the constant $C$ in (30) set $M=M_{0}$ and define $\Delta=\Delta_{0}$ in this case. Then $D=\left(D_{0}-1\right) M_{0}-C+\Delta_{0}-1$. On the other hand we know that when $M=M_{0}$, the head polynomial of the linear ODE factorizes into two polynomials, one of degree $D_{0}$ giving the true singularities of the linear ODE and one of degree $D_{a p p}$ whose zeros are all apparent singularities. Thus we can write $D=D_{0}+D_{a p p}$ which, combined with the solution $D=\left(D_{0}-1\right) M_{0}-C+\Delta_{0}-1$, yields

$$
C=\left(D_{0}-1\right) \cdot\left(M_{0}-1\right)-D_{a p p}+\Delta_{0}-2,
$$

giving a direct connection between $C$ and the apparent polynomial of the minimum order linear ODE. We remark further that $D_{a p p}$ is related to a (true) singularity exponent sum-rule. In the case of $\tilde{\chi}^{(5)}$ the exponents we have determined yield $D_{a p p}=1384$ and hence $C=(72-1)(33-1)-1384+\Delta_{0}-2=886+\Delta_{0} \geq 886$, which is consistent with the observed $C=900$. The details of this sum-rule calculation can be found in Appendix A.

The deviations $\Delta$ observed in Table 1 are quite small and a reasonable approximation to (30) is obtained by setting $\Delta=0$. Then (30) is both a specification of $N$, the minimum number of series terms needed to get a linear ODE, and a relationship between $M$ and $D$. We can use the latter to eliminate, say, $D$ and the former to find the minimum possible $N$. The result is that one should be exploring the region around $(M+1) /(D+1)=M_{0} / D_{0}$ to obtain the minimum $N$.

In earlier work [11, 12] we observed a "Russian-doll" structure for the linear differential equations for $\tilde{\chi}^{(3)}$ and $\tilde{\chi}^{(4)}$ and a similar inherited Russian-doll structure on the $n$-particle contributions of the "diagonal susceptibility" [9]. We conjecture for arbitrary $\tilde{\chi}^{(n)}$ a "strong" Russian-doll structure for the linear differential operator for $\tilde{\chi}^{(n)}$, which is to say that the linear differential operator for $\tilde{\chi}^{(n)}$ right-divides the linear differential operator for $\tilde{\chi}^{(n+2)}$. We can now verify this conjecture on $\tilde{\chi}^{(5)}$ and the results are shown in Table 2 .

Further, a stronger property amounts to saying that we actually have, in the decomposition of the linear differential operator for $\tilde{\chi}^{(n+2)}$, the linear differential operator for $\tilde{\chi}^{(n)}$ occuring as part of a direct sum. Such a reduction was found [10, $11,12]$ for the combinations $6 \cdot \tilde{\chi}^{(n+2)}-n \cdot \tilde{\chi}^{(n)}, n=1$ or 2 , and we now verify this conjecture for the case $n=3$. Detailed results that we referred to earlier are in Table 1. A summary of all the observed operator reductions appears in Table 2.

Some operator reduction data in Table 2 calls for explanation. Since the differential operator $L_{7}$ for $\tilde{\chi}^{(3)}$ [11], acting on the $\tilde{\chi}^{(5)}$ series gives an order 26 ODE (see sixth line in Table 2) and the minimal ODE for $6 \tilde{\chi}^{(5)}-3 \tilde{\chi}^{(3)}$ is of order 30, i.e. less than 33 (see ninth line in Table 2), one can conclude that this order 30 differential operator contains an order 4 differential operator occurring in the known $L_{7}$. From this order 4 differential operator, we focus here on the differential operators of order 1, which because they have no apparent singularities, are most effective in reducing the number of series coefficients that need to be generated. There are two such order 1 operators $\sharp$; their solutions are

$$
S_{1}=w /(1-4 w), \quad S_{2}=w^{2} /\left((1-4 w) \sqrt{1-16 w^{2}}\right),
$$

We have also found an order 1 operator whose solution

$$
S_{3}=w^{2} /(1-4 w)^{2}
$$

$\sharp$ They already occurred [10] as solutions of $L_{7}$, the differential operator for $\tilde{\chi}^{(3)}$. 
Table 2. Summary of results for various series. The equation for the size of the zero determinant is obtained from fits to data such as that shown in Table 1 for $\tilde{\chi}^{(5)}$. The last five columns are the data for the case that the zero determinant size is a minimum. A reduced difference in braces in column 7 appears in those cases where a constant is a solution of the ODE and the matrix being tested could have been taken as size $O \times(D+1)$ rather than $(O+1) \times(D+1)$.

The $\Phi_{H}^{(n)}$ series are the model integrals [14] (see (35)).

\begin{tabular}{|c|c|c|c|c|c|c|}
\hline Series & $N=D_{0} M+M_{0} D-C$ & $M$ & $D$ & $N_{M D}$ & $N$ & $\Delta$ \\
\hline$\tilde{\chi}^{(1)}$ & $1 M+1 D+1$ & 1 & 1 & 4 & 3 & 1 \\
$\tilde{\chi}^{(2)}$ & $1 M+2 D+2$ & 2 & 1 & 6 & 6 & 0 \\
$\tilde{\chi}^{(3)}$ & $12 M+7 D-40$ & 11 & 17 & 216 & 211 & 5 \\
$\tilde{\chi}^{(4)}$ & $7 M+9 D-36$ & 15 & 9 & 160 & 150 & $10(0)$ \\
$\tilde{\chi}^{(5)}$ & $72 M+33 D-900$ & 56 & 129 & 7410 & 7389 & 21 \\
$L_{7}\left(\tilde{\chi}^{(5)}\right)$ & $60 M+26 D+611$ & 54 & 131 & 7260 & 7257 & 3 \\
$6 \tilde{\chi}^{(3)}-\tilde{\chi}^{(1)}$ & $12 M+6 D-28$ & 10 & 17 & 198 & 194 & 4 \\
$6 \tilde{\chi}^{(4)}-2 \tilde{\chi}^{(2)}$ & $6 M+7 D-17$ & 13 & 8 & 126 & 117 & $9(0)$ \\
$6 \tilde{\chi}^{(5)}-3 \tilde{\chi}^{(3)}$ & $68 M+30 D-744$ & 52 & 120 & 6413 & 6392 & 21 \\
$L_{2}$ & $65 M+28 D-526$ & 50 & 117 & 6018 & 6000 & 18 \\
$L_{3}$ & $64 M+27 D-409$ & 49 & 117 & 5900 & 5886 & 14 \\
$\Phi_{H}^{(3)}$ & $10 M+5 D-21$ & 8 & 13 & 126 & 124 & 2 \\
$\Phi_{H}^{(4)}$ & $5 M+6 D-12$ & 9 & 6 & 70 & 69 & 1 \\
$\Phi_{H}^{(5)}$ & $45 M+17 D-277$ & 28 & 80 & 2349 & 2343 & 6 \\
$\Phi_{H}^{(6)}$ & $26 M+27 D-342$ & 48 & 39 & 1960 & 1959 & 1 \\
$\Phi_{H}^{(7)}$ & $145 M+49 D-1943$ & 92 & 257 & 23994 & 23990 & 4 \\
\hline
\end{tabular}

is a solution of the order 30 differential operator for $6 \tilde{\chi}^{(5)}-3 \tilde{\chi}^{(3)}$, but not a solution of the differential operator for $\tilde{\chi}^{(3)}$. Let us introduce the second order differential operator $\ddagger L_{2}$ which simultaneously annihilates $S_{1}$ and $S_{2}$ and the order 3 differential operator $L_{3}$ which annilates $S_{3}$ as well. When these act on the series $6 \tilde{\chi}^{(5)}-3 \tilde{\chi}^{(3)}$, the reductions in $N$ are shown in Table 2 (tenth and eleventh lines, labelled respectively as $L_{2}$ and $L_{3}$ ). The essential observation is that, although we found in our first computer runs that 6000 terms were not enough to get the linear ODE mod $p_{0}$ for $\tilde{\chi}^{(5)}$, this is now more than adequate to get the linear ODE modulo $p$ for $\tilde{\chi}^{(5)}$ for as many new primes $p$ as we wish to investigate.

\subsection{On the linear $O D E$ for $\tilde{\chi}^{(6)}$}

We do not have corresponding results for $\tilde{\chi}^{(6)}$ and it seems likely that obtaining long enough series in this case is beyond our presently available computing resources. We base this on the following very crude correspondence: determining the exact linear ODE for $\tilde{\chi}^{(3)}$ required a minimal series of about 220 terms. The exact ODE for $\tilde{\chi}^{(4)}$ required about 170 terms $\left(\right.$ in $x=w^{2}$ ). The ratio $N_{\tilde{\chi}^{(4)}} / N_{\tilde{\chi}^{(3)}} \simeq 170 / 220 \simeq 0.77$. There is a similar ratio for $\Phi_{H}^{(n)}$, integrals without the Fermionic factor introduced in [14]. From Table 2, we have $N_{\Phi^{(4)}} / N_{\Phi^{(3)}} \simeq 80 / 130 \simeq 0.62$ and $N_{\Phi^{(6)}} / N_{\Phi^{(5)}} \simeq$ 


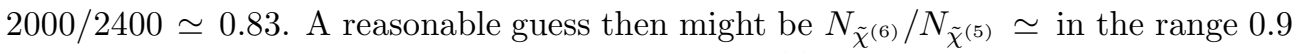
to 1.1. Although we generated 10000 terms for $\tilde{\chi}^{(5)}$ only about 7500 were actually required. Our guess is that about the same number will be required for $\tilde{\chi}^{(6)}$. Now our codes for $\tilde{\chi}^{(5)}$ and $\tilde{\chi}^{(6)}$ are such that 7500 terms for $\tilde{\chi}^{(6)}$ is roughly the equivalent in time to 15000 terms for $\tilde{\chi}^{(5)}$ and this means a running time for $\tilde{\chi}^{(6)}$ about $1.5^{4} \simeq 5$ times in excess of that taken for the 10000 terms of $\tilde{\chi}^{(5)}$. This might be reduced somewhat if we rely on some direct sum assumptions about the linear ODE for $\tilde{\chi}^{(6)}$ similar to those we have found work for $\tilde{\chi}^{(5)}$. On the other hand, a guess for a series length $N$ that is too small might leave us with no results whatsoever and thus we would probably want to err on the conservative side and require a calculation of $\tilde{\chi}^{(6)}$ with a run time cost as much as 10 times that for $\tilde{\chi}^{(5)}$.

\subsection{Singularities of the linear ODE for $\tilde{\chi}^{(5)}$ and Landau singularities}

From the linear ODE for $\tilde{\chi}^{(5)}$ obtained modulo a prime, one can easily reconstruct the singularity polynomials of the ODE as they appear at the highest derivative. These polynomials read

$$
\begin{aligned}
& w^{33} \cdot(1-4 w)^{22}(1+4 w)^{16}(1-w)^{4}(1+2 w)^{4}\left(1+3 w+4 w^{2}\right)^{4} \\
& (1+w)\left(1-3 w+w^{2}\right)\left(1+2 w-4 w^{2}\right)\left(1-w-3 w^{2}+4 w^{3}\right) \\
& \left(1+8 w+20 w^{2}+15 w^{3}+4 w^{4}\right)\left(1-7 w+5 w^{2}-4 w^{3}\right) \\
& \left(1+4 w+8 w^{2}\right)(1-2 w) .
\end{aligned}
$$

All these singularities, except $(1-2 w)$, have been predicted by the model integrals we introduced in [14]. The models considered in [13, 14] are integral representations (one-dimensional and multidimensional) which belong to the "Ising class" [25]. These integrals are holonomic and we obtained the linear ODE's of these sets of integrals through series expansions [13, 14]. In [14] a detailed analysis of the multiple integrals $\Phi_{H}^{(n)}$ was performed. These $n$-fold integrals correspond to removing the Fermionic factor $\left(G^{(n)}\right)^{2}$ in (5), so that

$$
\Phi_{H}^{(n)}(w)=\frac{1}{n !} \cdot\left(\prod_{j=1}^{n-1} \int_{0}^{2 \pi} \frac{d \phi_{j}}{2 \pi}\right)\left(\prod_{j=1}^{n} y_{j}\right) \cdot \frac{1+\prod_{i=1}^{n} x_{i}}{1-\prod_{i=1}^{n} x_{i}} .
$$

We obtained (after eliminating the apparent singularities) the following polynomial factors for the head polynomial of the linear ODE's satisfied by the $\Phi_{H}^{(n)}$, expressed in terms of Chebyshev polynomials of the first and second kind [14]:

$$
\begin{array}{ll}
T_{2 p_{1}}(1 / 2 w+1)= & T_{n-2 p_{1}-2 p_{2}}(1 / 2 w-1), \\
0 \leq p_{1} \leq[n / 2], & 0 \leq p_{2} \leq[n / 2]-p_{1},
\end{array}
$$

and the polynomial arising from the elimination of $z$ in:

$$
\begin{aligned}
& T_{n_{1}}(z)-T_{n_{2}}\left(\frac{4 w-z}{1-4 w z}\right)=0, \\
& T_{n_{1}}\left(\frac{1}{2 w}-z\right)-T_{n_{2}}\left(\frac{1}{2 w}-\frac{4 w-z}{1-4 w z}\right)=0, \\
& U_{n_{2}-1}(z) \cdot U_{n_{1}-1}\left(\frac{1}{2 w}-\frac{4 w-z}{1-4 w z}\right) \\
& \quad-U_{n_{2}-1}\left(\frac{1}{2 w}-z\right) \cdot U_{n_{1}-1}\left(\frac{4 w-z}{1-4 w z}\right)=0,
\end{aligned}
$$




$$
\begin{array}{ll}
n_{1}=p_{1}, & n_{2}=n-p_{1}-2 p_{2}, \\
0 \leq p_{1} \leq n, & 0 \leq p_{2} \leq\left[\left(n-p_{1}\right) / 2\right]
\end{array}
$$

Our motivation was to obtain "good candidates" for the factors in the head polynomial of the linear ODE of $\tilde{\chi}^{(n)}$, expecting that the Fermionic factor $\left(G^{(n)}\right)^{2}$ may not introduce "too many additional singularities".

There is also the possibility of singularity cancellation. Indeed, in the even simpler integral in which the $R^{(n)}$ factor (7) is dropped from (35), all the singularities predicted in [16] are seen; these are the singularities (36) but with the even $2 p_{1}$ replaced by an integer that can also be odd. Thus the inclusion of $R^{(n)}$ has eliminated a whole class of singularities. The presence of $\left(G^{(n)}\right)^{2}$, at least in $\tilde{\chi}^{(5)}$, has been much less dramatic and led only to the one extra factor $(1-2 w)$ in $(34)$.

To get a better understanding of the effect of $\left(G^{(n)}\right)^{2}$ we revisit the Landau singularity approach in Appendices B through E but performing the calculations on the $(n-1)$-fold integrals $(5)$ rather than the original $(2 n-2)$-fold integrals [2] that was the basis of calculations in [14]. The new approach detailed in Appendix B confirms our previous Landau singularity calculations [13, 14], the difference being in the number of integration variables considered. The original representation for $\tilde{\chi}^{(n)}$ given in [2] is an integral over two sets of $(n-1)$ independent phases $\phi_{i}, \zeta_{j}$ with an integrand that is symmetric under the interchange of these sets. Integrating out one set to arrive at (5) has obviously broken this symmetry but a vestige of it remains, such that for every combination of the $\zeta_{j}, \phi_{i}$ leading to a singularity there is another set $\phi_{j}, \zeta_{i}$ obtained by $\zeta \leftrightarrow \phi$ interchange that leads to the same singularity. In addition another symmetry arises such that, for a given $\phi_{i}, \zeta_{i}$ combination, the Landau conditions allow the reversed combination $-\phi_{i},-\zeta_{i}$ explaining the $n \rightarrow n-2 m$ replacement singularities seen to occur in the analysis of [14]. Our analysis proves that for the $\Phi_{H}^{(n)}$ there are no singularities other than those given by (36) and (37). We also show that $\left(G^{(n)}\right)^{2}$ neither reintroduces the singularities cancelled by the $R^{(n)}$ nor leads to further cancellation and thus the singularities of $\Phi_{H}^{(n)}$ are included in the singularities of $\tilde{\chi}^{(n)}$. The $\left(G^{(n)}\right)^{2}$ factor has a dramatic effect on the exponents of the singularities and a power counting argument is given in Appendix E to predict the exponents at all singularities. Those calculations are not intended to cover all possible contingencies and while many predicted exponents are observed there remain a number of "anomalous" cases.

Our analysis confirms the fact that the Landau singularities of $\tilde{\chi}^{(n)}$, at least those given by $\Phi_{H}^{(n)}$, are included in the set of singularities of $\tilde{\chi}^{(n+2 k)}, k \geq 1$. This is exactly what we have found for the linear ODE for $\tilde{\chi}^{(5)}$. The first line in (34) corresponds to the singularities occurring in the linear ODE for $\tilde{\chi}^{(3)}$. This is a necessary condition for $\tilde{\chi}^{(3)}$ to be embedded in $\tilde{\chi}^{(5)}$. However this comes with an important caveat. We prove in Appendix B that none of the embedded singularities can lie on the $|s|=1$ boundary of the principal disc of the $\tilde{\chi}^{(n)}$ function defined by the integral (5). We also show that of all the different classes of singularities, only those we call Case 2 irreducible singularities in Appendix B lie on $|s|=1$ of the principal disc. These correspond to the singularities derived in $[3,4]$ and are elsewhere called the Nickelian or circle singularities. The importance of this lies in the fact that we cannot expect cancellation between different singularities on the principal disc and thus the elimination of $|s|=1$ as a natural boundary.

While some aspects of the effect of $\left(G^{(n)}\right)^{2}$ have been determined, a complete 
Landau singularity analysis of $\tilde{\chi}^{(n)}$ has not been done; in particular we cannot definitively state whether the $(1-2 w)$ factor in (34) does or does not identify with a Landau singularity of $\tilde{\chi}^{(5)}$. However, a toy integral intermediate between $\Phi_{H}^{(n)}$ and $\tilde{\chi}^{(n)}$ is discussed in Appendix D and provides an example in which it can be shown that the ODE describing an integral has more singularities than the integral. The analogy to $\tilde{\chi}^{(n)}$ is sufficiently close that we believe it is likely that $(1-2 w)=0$ is not a singularity of $\tilde{\chi}^{(5)}$. Clearly it is of interest to know whether there are such additional singularities in the ODE for $\tilde{\chi}^{(n)}$ for larger $n$ and whether they are also on $|s|=1$.

Appendix $\mathrm{C}$ lists, for the first few $n$ of $\Phi_{H}^{(n)}$, the singularities corresponding to $(36,37)$ and also derived in Appendix B. In the next section we will see whether these singularities appear in the analysis of our long series with exact coefficients.

\section{Diff-Padé analysis of the long series for $\tilde{\chi}^{(5)}$ and $\tilde{\chi}^{(6)}$}

We present, in this section, a diff-Padé analysis of our long 2000 coefficient series (these are the actual series coefficients, not the coefficients modulo a prime, for which we have longer series) in floating point form, in order to obtain the singularities (together with their associated exponents) that should occur in the corresponding linear ODE's.

\subsection{Singularities and indicial exponents of the linear $O D E$ of $\tilde{\chi}^{(5)}$}

We begin with the series for $\tilde{\chi}^{(5)}$, for which we have obtained the linear ODE (modulo a prime) and have recognized all the singularities as given in (34). Our calculations provides a check on whether or not a diff-Padé analysis on a series too short to find the exact ODE can nevertheless yield enough information to locate the singularities precisely and determine the associated local exponents accurately.

A diff-Padé analysis with just 400 coefficients (using approximating linear ODE's of order ten or eleven) already confirms, with 36 digit accuracy, the occurrence of the singularities given by the roots of the factors

$$
(1+w)\left(1-3 w+w^{2}\right)\left(1+2 w-4 w^{2}\right),
$$

which are the Nickelian singularities labelled as $P\left({ }^{2} 5\right)$, and the roots of the factors

$$
\left(1-7 w+5 w^{2}-4 w^{3}\right)\left(1+8 w+20 w^{2}+15 w^{3}+4 w^{4}\right),
$$

with twelve digit accuracy. The roots of the factors

$$
(1-w)(1+2 w)\left(1-w-3 w^{2}+4 w^{3}\right)
$$

are confirmed with three or four digit accuracy, and, finally, the roots of the factor $1+4 w+8 w^{2}$ are obtained with just one digit accuracy. The roots of $1+3 w+4 w^{2}$ do not yet appear in the analysis using 400 coefficients.

A generalized diff-Padé analysis is conducted by steadily increasing the order of the linear ODE and the degree of the polynomials, while looking for the roots which stabilise with increasing accuracy. For instance, using 1250 coefficients, the roots (39) now appear with fifteen digit accuracy, and similarly for the other roots, the accuracy increases. While the roots of the factor $1+3 w+4 w^{2}$ are not yet observed, we see

ๆ We call diff-Padé the type of analysis detailed in [11]. For a given number of terms $N$ of the series, there is a linear ODE of order $q$ that reproduces the first $N-q$ terms but may fail for subsequent coefficients. The same analysis, called "method of differential approximants" was described in [26]. 
the emergence of a new polynomial not found among the set of singularities of $\Phi_{H}^{(5)}$ : the singularity $w=+1 / 2$ is actually observed with twelve digit accuracy.

Further increasing the degrees and the order of the linear ODE to fully utilise the 1980 coefficients at our disposal, the accuracy is dramatically improved. The roots (39) are now confirmed up to 67 digits, the two complex roots of polynomial $1-w-3 w^{2}+4 w^{3}$ are observed with more than 51 correct digits, and the real root is observed with more than 7 correct digits. The roots of the factor $1+4 w+8 w^{2}$ are now observed with 17 digit accuracy. The roots of $(1-w)(1+2 w)$ are seen with 5 correct digits for $w=1$ and 7 correct digits for $w=-1 / 2$. Finally, the roots of $1+3 w+4 w^{2}$ are seen with 4 digit accuracy, and the "new" $(1-2 w)$ factor is observed with 27 correct digits. We summarise this discussion in Table 3.

Table 3. This table shows the number of significant digits found, in the case of each singularity of $\tilde{\chi}^{(5)}$, from a differential approximant (diff-Padé) analysis of series of length, respectively, 400, 1250 and 1980 terms.

\begin{tabular}{|c|c|c|c|c|}
\hline Label of singularity & Associated polynomial & 400 & 1250 & 1980 \\
\hline$P\left({ }^{2} 5\right)$ & $(1+w)$ & 36 & & \\
$P\left({ }^{2} 5\right)$ & $\left(1+2 w-4 w^{2}\right)$ & 36 & & \\
$P\left({ }^{2} 5\right)$ & $\left(1-3 w+w^{2}\right)$ & 36 & & \\
$P\left({ }^{3} 5_{1,4}\right)$ & $\left(1+8 w+20 w^{2}+15 w^{3}+4 w^{4}\right)$ & 12 & 15 & 67 \\
$P\left({ }^{3} 5_{3,2}\right)$ & $\left(1-7 w+5 w^{2}-4 w^{3}\right)$ & 12 & 15 & 67 \\
$P\left({ }^{4} 5_{4,1}\right)$ & $\left(1-w-3 w^{2}+4 w^{3}\right)$ & 4 & 15 & 51 \\
$P\left({ }^{4} 5_{3,2}\right)$ & $\left(1+4 w+8 w^{2}\right)$ & - & 8 & 17 \\
$P\left({ }^{5} 5 / 23\right)$ & $(1+2 w)$ & 3 & 5 & 7 \\
$P\left({ }^{5} 5 / 23\right)$ & $(1-w)$ & 3 & 3 & 5 \\
$P\left({ }^{5} 5 /{ }^{3} 3_{1,2}\right)$ & $\left(1+3 w+4 w^{2}\right)$ & - & - & 4 \\
Unknown & $(1-2 w)$ & - & 12 & 27 \\
\hline
\end{tabular}

These diff-Padé calculations are, in fact, sufficiently robust to allow us to predict the minimum multiplicity of some singularities. When a given singularity (say $w=1$ ) is observed, it is put in the head polynomial for a second run using more coefficients. If this singularity appears again it must be a double root (one exact root $w=1$ and a second root $w \simeq 1$ observed numerically with sufficient accuracy). Next the factor $(1-w)^{2}$ is included in the head polynomial for another run and so on until no further occurrences of the given root are found.

With the number of series coefficients at hand we find for the head polynomial of the ODE the following factors occurring with the indicated multiplicity:

$$
(1+2 w)^{4} \cdot(1-w)^{4} \cdot\left(1+3 w+4 w^{2}\right)^{2},
$$

while all other roots (except $1-16 w^{2}$ ) occur with multiplicity one. We are very close to the exact multiplicities of the exact linear ODE (see (34)).

This knowledge can then be used, in a kind of converging procedure, to improve the accuracy of our diff-Padé calculations. Having a totally unambiguous location of the singularities and a minimum value for the multiplicities, we revisit the diff-Padé calculations using the following Ansatz for the polynomial in front of the $i^{\text {th }}$ derivative

$$
\begin{aligned}
w^{i-1} \cdot & \left(1-16 w^{2}\right)^{i-2} \cdot((1+2 w) \cdot(1-w))^{i-q+4} \\
& \times\left(1+3 w+4 w^{2}\right)^{i-q+2} \cdot P(w)^{i-q+1} \cdot Q_{i}(w)
\end{aligned}
$$


with $P(w)$ containing the other singularities with multiplicity one:

$$
\begin{aligned}
P(w) & =(1+w)\left(1-3 w+w^{2}\right)\left(1+2 w-4 w^{2}\right)\left(1+4 w+8 w^{2}\right) \\
& \times(1-2 w)\left(1-7 w+5 w^{2}-4 w^{3}\right)\left(1-w-3 w^{2}+4 w^{3}\right) \\
& \times\left(1+8 w+20 w^{2}+15 w^{3}+4 w^{4}\right)
\end{aligned}
$$

Here $q$ is the order of the linear ODE and the index $i=0, \cdots, q$ denotes the successive derivatives in the linear ODE (the actual exponents in the Ansatz are zero when a negative value is encountered). The $Q_{i}(w)$ are unknown polynomials, and $Q_{q}(w)$ at the highest derivative is included in order to handle the expected apparent singularities.

The Ansatz (41) is then used in a diff-Padé analysis to obtain in floating point form, at each singularity, the associated critical exponents as roots of the indicial equation. Our findings are listed in Table 4. Let us explain how we display our results. Consider for instance the singularity $1-w=0$. The successive roots of the indicial equation appear as the integers $0,1, \cdots, q-5$ (which is by construction) together with the integers $2,3,3,4$. The results for this case will be displayed as $2,3^{2}, 4$, but note that the roots 2 and 4 both appear twice, and the root 3 appears three times. Recall that the roots of the indicial equations appear in floating point form and we recognize these roots as being integers or half integers. The accuracy of the indicial exponents can be as low as three correct digits as is the case at the singularities given by $1+3 w+4 w^{2}=0$.

Table 4. Singularity exponent list for $\tilde{\chi}^{(5)}$, based on the exact (modulo a prime) linear ODE compared to those found by the (floating-point) diff-Padé analysis. The mod prime exponent list is followed by a number in braces, which is the sum in (A.7) of Appendix A. A final column gives the expected "normal" exponents using (E.1).

\begin{tabular}{|c|c|c|c|}
\hline $\begin{array}{c}\text { Singularity } \\
\text { Polynomial }\end{array}$ & $\begin{array}{c}\text { Exponents (from } \\
\text { mod prime analysis })\end{array}$ & $\begin{array}{c}\text { Exponents } \\
\text { (diff-Padé) }\end{array}$ & $\begin{array}{c}\text { Exponents } \\
\text { (E.1) }\end{array}$ \\
\hline$w^{33}$ & $1^{5}, 2^{4}, 3^{4}, 4^{3}, 5^{3}, 6^{3}, 7^{2}, 8^{2}, 9^{2}$, & & - \\
$10,12^{2}, 15,25,(192)$ & & -1 \\
$(1-4 w)^{22}$ & $-2,-7 / 4,-3 / 2,-5 / 4,-1^{3},-1 / 2,0^{4}$, & $-3 / 2,-1,0^{4}$ & \\
& $1 / 2,1^{2}, 2^{2}, 3,4,5,6,7,(549 / 2)$ & & \\
$(1+4 w)^{16}$ & $-1,-1 / 2,0^{4}, 1 / 2,1^{2}, 3 / 2,2^{2}, 3^{2}$, & $-1 / 2,0^{4}, 1^{2}$ & - \\
& $4,5,(315 / 2)$ & $0^{3}, 1^{4}, 2$ & 3 \\
$\left(1 / w^{19}\right.$ & $0^{3}, 1^{4}, 2^{2}, 3^{3}, 4^{2}, 5^{2}, 6,7,8,(56+91)$ & $2,5 / 2,3^{2}$ & 3 \\
$(1-w)^{4}$ & $2,5 / 2,3^{2},(41 / 2)$ & $2,3^{2}, 4$ & 1 \\
$\left(1+3 w+4 w^{2}\right)^{4}$ & $2,3^{2}, 4,(22)$ & 11 & 11 \\
$(1+w)$ & $0,1^{2}, 2,(14) \times 2$ & 11 & 11 \\
$\left(1+2 w-4 w^{2}\right)$ & $11,(12)$ & 7 & 11 \\
$\left(1-3 w+w^{2}\right)$ & $11,(12) \times 2$ & 5 & 7 \\
$\left(1-7 w+5 w^{2}-4 w^{3}\right)$ & $11,(12) \times 2$ & 7 & 7 \\
$\left(1-w-3 w^{2}+4 w^{3}\right)$ & $7,(8)$ & 5 & 5 \\
$\left(1-2 w+8 w^{2}\right)$ & $5,(6) \times 3$ & $7 / 2$ & - \\
\hline
\end{tabular}

Since we have obtained the exact linear ODE for $\tilde{\chi}^{(5)}$ this case provides a valuable 
test of our diff-Padé analysis. Furthermore, the Landau singularity analysis has been extended, in Appendix E, to include a power counting argument for the exponents at each singularity. All these results, the exponents from a diff-Padé analysis on 2000 terms, the exponents from the (modulo a prime) linear ODE, and those derived in Appendix E are displayed together in Table 4.

Even if we did not know the exact (modulo a prime) linear ODE of $\tilde{\chi}^{(5)}$, our diff-Padé analysis can provide accurate information about the exact ODE. We note that the indicial exponents found above are accurate enough that we can be confident in their exact values, but the set of exponents may be incomplete. Let us consider the case $1-2 w=0$ to show what we mean. This factor was taken with a multiplicity of one in the head polynomial and the roots of the indicial equation show up as $0,1, \cdots, q-2,7 / 2$. Assume that in the exact linear ODE, the multiplicity is two. Then, the roots of the indicial equation will be $0,1, \cdots, q-3,7 / 2$ plus an unknown exponent. If we had more series coefficients, with a further increase of the order and the degrees, this unknown indicial exponent may be obtained as another half integer, the exponent $7 / 2$ or an integer. The dominant singular behavior at $w=1 / 2$ would change accordingly.

The comparison with the exact ODE results shows that the diff-Padé analysis on only 2000 terms (which are insufficient to encode the linear ODE for $\tilde{\chi}^{(5)}$ ) is able to correctly give all the local exponents for the singularities together with the correct multiplicity. The singularity polynomial $\left(1+3 w+4 w^{2}\right)$ was used in the head polynomial of the linear ODE with a multiplicity of two instead of the correct multiplicity four. One should then obtain two local exponents. The missing local exponent was obtained as 0.87 instead of 1 .

These results give us considerable confidence that our numerical analysis of $\tilde{\chi}^{(6)}$ in the following subsection, and of higher order susceptibility components subsequently, are completely correct.

\subsection{Singularities and indicial exponents of the linear ODE of $\tilde{\chi}^{(6)}$}

Similar calculations to those detailed in the previous section for $\tilde{\chi}^{(5)}$ have been performed for $\tilde{\chi}^{(6)}$ in the variable $x=w^{2}$.

In a diff-Padé analysis, increasing the order of the linear ODE and the degree of the polynomials, the singularities predicted by the $\Phi_{H}^{(6)}$ model (labelled as $P\left({ }^{2} 6\right) P\left({ }^{3} 6_{4,2}\right) P\left({ }^{4} 6_{5,1}\right)$ and $\left.P\left({ }^{2} 4\right)\right)$ are obtained with increasing accuracy.

We should note that the additional singularity $1-2 w=0$, occurring for $\tilde{\chi}^{(5)}$, was seen in our diff-Padé analysis before we obtained the exact (modulo a prime) linear ODE. Let us detail for $\tilde{\chi}^{(6)}$ how our numerical procedure proceeds. First we check whether the "candidate" singularities appear as roots of the head polynomials, and we also check whether some of the other roots stabilize as the number of terms and the order of the linear ODE increases. If so this root is a true singularity. Next the well confirmed "candidate" singularities are put into the head polynomial and another run is carried out with more terms to confirm the "new singularity".

Table 5 shows the results of three specific runs. The third column shows results from a run using 387 terms with order 12 ODE. An unknown (with respect to our candidates) singularity is seen to stabilize around a value consistent with $1-8 x=0$ to an accuracy of 3 digits. Keeping the same number of terms but increasing the order to 16 this root would change if it were a root of the apparent polynomial. The results in the fourth column show that the new unknown singularity remains and is seen with 
4 digit accuracy with respect to its exact value. Finally all the other singularities are put into the head polynomial and the fifth column shows the results for an order 31 analysis using 997 terms. These calculations show the existence of a new singularity at $x=1 / 8$ and thus arising from the factor $1-8 w^{2}=0$ in the head polynomial of the true ODE.

Table 5. This table shows the number of significant digits found, in the case of each singularity of $\tilde{\chi}^{(6)}$, from a diff-Padé analysis $\left(x=w^{2}\right)$.

\begin{tabular}{|c|c|c|c|c|}
\hline $\begin{array}{c}\text { Singularity } \\
\text { Label }\end{array}$ & $\begin{array}{c}\text { Singularity } \\
\text { Polynomial }\end{array}$ & $\begin{array}{c}387 \text { terms, } \\
\text { order } 12\end{array}$ & $\begin{array}{c}387 \text { terms, } \\
\text { order } 16\end{array}$ & $\begin{array}{c}997 \text { terms, } \\
\text { order } 31\end{array}$ \\
\hline$P\left({ }^{2} 6\right)$ & $1-x$ & 28 & 29 & \\
$P\left({ }^{5} 6 /{ }^{2} 4\right)$ & $1-4 x$ & 30 & 30 & \\
$P\left({ }^{2} 6\right)$ & $1-9 x$ & 30 & 30 & \\
$P\left({ }^{3} 6_{4,2}\right)$ & $1-25 x$ & 13 & 14 & \\
$P\left({ }^{3} 6_{4,2}\right)$ & $1-x+16 x^{2}$ & 8 & 10 & \\
$P\left({ }^{4} 6_{5,1}\right)$ & $1-10 x+29 x^{2}$ & 10 & 12 & \\
Unknown & $1-8 x$ & 3 & 4 & 26 \\
\hline
\end{tabular}

Various runs (with linear ODE's ranging in order from 26 to 31 ) show that the factor $(1-4 x)$ occurs with a power five which leads us to the following Ansatz:

$$
x^{i-1} \cdot(1-16 x)^{i-2} \cdot(1-4 x)^{i-q+5} \cdot P(x)^{i-q+1} \cdot Q_{i}(x)
$$

for the polynomials in front of the $i^{t h}$ derivative in the linear ODE where $P(x)$ reads:

$$
\begin{aligned}
P(x) & =(1-x)(1-9 x)(1-25 x)\left(1-x-16 x^{2}\right) \\
& \times\left(1-10 x+29 x^{2}\right)(1-8 x) .
\end{aligned}
$$

The singularities and corresponding exponents for $\tilde{\chi}^{(6)}$ are summarised in Table 6 , and we also show the exponents predicted by the local analysis given in Appendix E.

Table 6. Singularity exponent list for $\tilde{\chi}^{(6)}$ found by the (floating-point) diff-Padé analysis together with the expected "normal" exponents (E.1).

\begin{tabular}{|c|c|c|c|}
\hline $\begin{array}{c}\text { Singularity } \\
\text { Label }\end{array}$ & $\begin{array}{c}\text { Singularity } \\
\text { Polynomial }\end{array}$ & $\begin{array}{c}\text { Exponents } \\
\text { (diff-Padé) }\end{array}$ & $\begin{array}{c}\text { Exponents } \\
\text { (E.1) }\end{array}$ \\
\hline & $x$ & $0,-1^{2},-1 / 2$ & - \\
& $1-16 x$ & $-3 / 2,-1,0^{5}, 1$ & -1 \\
& $1 / x$ & $-1^{2}, 0^{2},-1 / 2^{2}, 1 / 2^{6}$ & - \\
$P\left({ }^{2} 6\right)$ & $1-x$ & $33 / 2$ & $33 / 2$ \\
$P\left({ }^{5} 6 /{ }^{2} 4\right)$ & $1-4 x$ & $11 / 2,13 / 2^{2}, 15 / 2,33 / 2$ & $13 / 2$ \\
$P\left({ }^{2} 6\right)$ & $1-9 x$ & $33 / 2$ & $33 / 2$ \\
$P\left({ }^{3} 6_{4,2}\right)$ & $1-25 x$ & $17 / 2$ & $17 / 2$ \\
$P\left({ }^{3} 6_{4,2}\right)$ & $1-x+16 x^{2}$ & $17 / 2$ & $17 / 2$ \\
$P\left({ }^{4} 6_{5,1}\right)$ & $1-10 x+29 x^{2}$ & $23 / 2$ & $23 / 2$ \\
Unknown & $1-8 x$ & 7 & - \\
\hline
\end{tabular}

Again we see that with some 1600 terms (which are insufficient to encode the linear ODE for $\tilde{\chi}^{(6)}$ ) the diff-Padé analysis is able to give the correct exponents in agreement 
with Appendix E. Depending on the multiplicity of the singularity polynomial put into the linear ODE, the exponents may be incomplete. Again we find rational exponents for all the singularities of the linear ODE for $\tilde{\chi}^{(6)}$.

From our diff-Padé calculations we thus have confirmation that the singularities encoded in the head polynomial of the as yet unknown linear ODE for $\tilde{\chi}^{(6)}$ are (at least) the ones of $\Phi_{H}^{(6)}$ together with the roots of a new polynomial $1-8 w^{2}$. Note that these $1-8 w^{2}=0$ additional singularities lie on the unit circle $|s|=1$. At this stage, and since all the series coefficients of $\tilde{\chi}^{(6)}$ have been used, one may ask if there are other singularities like $w^{2}=1 / 8$ "still to be discovered"? Without the exact linear ODE of $\tilde{\chi}^{(6)}$ we cannot give a definitive answer to this question.

\section{Diff-Padé analysis for $\tilde{\chi}^{(n)}, n \geq 7$}

It is known [8] that the first non-zero coefficients in $\tilde{\chi}^{(n)}$ appear at order $n^{2}$ in $w$. The high- or low-temperature series for $\tilde{\chi}$ up to $N=2000$ then contains contributions from all the odd, respectively even, $\tilde{\chi}^{(n)}$ up to $n=\sqrt{N}$.

Since we know the first 2000 series coefficients for $\tilde{\chi}^{(5)}$ and the first 1630 coefficients for $\tilde{\chi}^{(6)}$ as well as the series for $\tilde{\chi}^{(n)}, n \leq 4$, up to an arbitrary number of coefficients, one may ask whether the total $\tilde{\chi}$ with these lower $\tilde{\chi}^{(n)}$ terms removed, can yield any information about the singularities that should occur in the linear ODE of $\tilde{\chi}^{(n)}, n \geq 7$ ? As far as $\tilde{\chi}^{(5)}$ and $\tilde{\chi}^{(6)}$ are concerned and in view of the "limited" analysis done here for $\tilde{\chi}^{(n)}, n \geq 7$, our conclusion is that there are no new singularities that are not in the "known" set (i.e. irrespective of the index $n$ ).

\subsection{High temperature analysis}

The diff-Padé analysis of the long high-temperature series

$$
\tilde{\chi}_{H}-\tilde{\chi}^{(1)}-\tilde{\chi}^{(3)}-\tilde{\chi}^{(5)}=128 w^{49}+25088 w^{51}+\cdots
$$

amounts to looking for the singularities of a "linear ODE" approximation to the (infinite non-holonomic) sum, $\tilde{\chi}^{(7)}+\tilde{\chi}^{(9)}+\tilde{\chi}^{(11)}+\cdots$, as roots of the head polynomial of a given diff-Padé approximant. These singularities occur grouped together (that is to say we find several singularities simultaneously) and each singularity can be attributed to a given $\tilde{\chi}^{(n)}$ according to the polynomials given in Appendix C, i.e. arising from the $\Phi_{H}^{(n)}$ model.

With an order eight linear ODE and 312 terms, the roots of the following polynomials (besides $1-16 w^{2}=0$ ) are recognized:

$$
\begin{aligned}
& P_{1}: 1+2 w-8 w^{2}-8 w^{3}=0, \\
& P_{2}: 1+2 w-w^{2}-w^{3}=0, \\
& P_{3}: 1-5 w+6 w^{2}-w^{3}=0 .
\end{aligned}
$$

These polynomials can be identified with all the Nickelian singularities corresponding to $\tilde{\chi}^{(7)}$. The roots of the polynomial $P_{1}$ are obtained with 5,10 and 12 correct digits, while two roots of the polynomial $P_{2}$ are obtained with 3 and 9 correct digits.

A summary of various runs using 500, 900 and 1956 terms is given in Table 7 , where we display the number of significant digits found, in the case of each singularity of the partial high-temperature susceptibility (43). The Ansatz for the linear ODE search is to include only the factor $\left(1-16 w^{2}\right)^{i}$, where $i$ is a positive 
Table 7. This table shows the number of significant digits found for each singularity of the partial high-temperature susceptibility (43) using a diff-Padé analysis. The singularities equal those predicted from $\Phi_{H}^{(n)}$ as occurring in $\tilde{\chi}^{(n)}$. The second column gives the polynomial factor, the zeros of which corresponds to the singularity locations. Subsequent pairs of columns give, firstly, the number of significant digits found for that singularity, and secondly the order of the linear ODE for which this singularity was found.

\begin{tabular}{|c|c|c|c|c|c|c|c|}
\hline & & \multicolumn{2}{|c|}{500 terms } & \multicolumn{2}{|c|}{900 terms } & \multicolumn{2}{|c|}{1956 terms } \\
\hline$n$ & polynomial & Digits & Order & Digits & Order & Digits & Order \\
\hline 7 & $1-5 w+6 w^{2}-w^{3}$ & 18 & 12 & 33 & 19 & 50 & 18 \\
\hline 7 & $1+2 w-w^{2}-w^{3}$ & 16 & 11 & 28 & 15 & 58 & 18 \\
\hline 7 & $1+2 w-8 w^{2}-8 w^{3}$ & 18 & 11 & 34 & 15 & 58 & 18 \\
\hline 9 & $1-w$ & - & & - & & $\overline{15}$ & 14 \\
\hline 9 & $1+2 w$ & - & & 6 & 13 & 30 & 16 \\
\hline 9 & $1+3 w-w^{2}$ & 5 & 11 & 16 & 15 & 45 & 15 \\
\hline 9 & $1-6 w+9 w^{2}-w^{3}$ & 7 & 12 & 20 & 13 & 45 & 18 \\
\hline 9 & $1-3 w^{2}-w^{3}$ & - & & 8 & 15 & 30 & 18 \\
\hline 9 & $1-12 w^{2}+8 w^{3}$ & - & & 14 & 14 & 40 & 20 \\
\hline 11 & $1-9 w+28 w^{2}-35 w^{3}+15 w^{4}-w^{5}$ & - & & 7 & 13 & 30 & 18 \\
\hline 11 & $1+2 w-5 w^{2}-2 w^{3}+4 w^{4}-w^{5}$ & - & & - & & 23 & 17 \\
\hline 11 & $1+2 w-16 w^{2}-24 w^{3}+48 w^{4}+32 w^{5}$ & - & & - & & 20 & 20 \\
\hline 13 & $1-11 w+\cdots+w^{6}$ & - & & - & & 16 & 16 \\
\hline 13 & $1+2 w-20 w^{2}+\cdots-64 w^{6}$ & - & & - & & 7 & 18 \\
\hline 15 & $1+2 w-4 w^{2}$ & - & & - & & 5 & 14 \\
\hline 15 & $1-9 w+\cdots+w^{4}$ & - & & - & & 5 & 14 \\
\hline 7 & $1+12 w+54 w^{2}+\cdots+4 w^{6}$ & - & & $\overline{6}$ & 13 & 12 & 17 \\
\hline 7 & $1-3 w-10 w^{2}+\cdots-16 w^{8}$ & - & & - & & 4 & 12 \\
\hline 9 & $1+16 w+104 w^{2}+\cdots+4 w^{8}$ & - & & - & & 7 & 17 \\
\hline
\end{tabular}

integer discussed in the previous section. The first block of singularities correspond to Nickelian singularities, given as Case 2 in Appendix C.

Again we have confirmation that some of the singularities of the linear ODE of $\Phi_{H}^{(n)}$ are actually singularities of the linear $O D E$ of the $\tilde{\chi}^{(n)} \ddagger$. From Table 7 we note that the first singularity polynomials to appear are the Nickelian ones. All the Nickelian singularities for $n=7$ and $n=9$ are confirmed. Of the four singularity polynomials for $n=11$, one is missing and among the five singularity polynomials for $n=13$, three are missing. We also note that once the Nickelian singularities for a given $n$ have appeared the non-Nickelian ones begin to show up.

$\ddagger$ Note however that without an "exact" series for each $\tilde{\chi}^{(n)}$, one cannot safely attribute, for instance, the non-Nickelian singularities polynomial

$$
\left(1+16 w+104 w^{2}+352 w^{3}+660 w^{4}+672 w^{5}+336 w^{6}+63 w^{7}+4 w^{8}\right)
$$

to $\tilde{\chi}^{(9)}$. These singularities may well come from $\tilde{\chi}^{(7)}$, but not be predicted by $\Phi_{H}^{(7)}$. 


\subsection{Low temperature analysis}

Similarly, the diff-Padé analysis of the long low-temperature series $\left(x=w^{2}\right)$

$$
\tilde{\chi}_{L}-\tilde{\chi}^{(2)}-\tilde{\chi}^{(4)}-\tilde{\chi}^{(6)}=256 x^{32}+65536 x^{33}+\cdots
$$

amounts to looking for the singularities of the "linear ODE" of the (infinite) sum, $\tilde{\chi}^{(8)}+\tilde{\chi}^{(10)}+\tilde{\chi}^{(12)}+\cdots$, as roots of the head polynomial of the diff-Padé approximant.

The Ansatz for the ODE search is to include only the factor $(1-16 x)^{i}$. Here we show the results in Table 8 for two orders fully utilising the series coefficients at our disposal. The first block of singularities correspond to Nickelian singularities given as Case 2 in Appendix C.

Table 8. The number of significant digits found for each singularity of the partial low-temperature susceptibility (44) using a diff-Padé analysis. The singularities are those predicted from $\Phi_{H}^{(n)}$ to occur in $\tilde{\chi}^{(n)}$. The second column gives the polynomial factor whose roots give the singularity locations. Subsequent columns give the number of significant digits found for that singularity at orders 14 and 20 .

\begin{tabular}{|c|c|c|c|}
\hline$n$ & polynomial & Order 14 & Order 20 \\
\hline $8,12,16$ & $1-4 x$ & 69 & 72 \\
8,16 & $1-2 x$ & 51 & 52 \\
8,16 & $1-8 x$ & 74 & 75 \\
8,16 & $1-12 x+4 x^{2}$ & 73 & 79 \\
\hline 10,12 & $1-x$ & 21 & 24 \\
10 & $1-5 x$ & 43 & 46 \\
10 & $1-7 x+x^{2}$ & 42 & 44 \\
10 & $1-12 x+16 x^{2}$ & 49 & 53 \\
10 & $1-15 x+25 x^{2}$ & 55 & 59 \\
\hline 12 & $1-9 x$ & 12 & 16 \\
12 & $1-3 x$ & 10 & 12 \\
12 & $1-12 x$ & 9 & 13 \\
12 & $1-14 x+x^{2}$ & 35 & 40 \\
12 & $1-8 x+4 x^{2}$ & 6 & 6 \\
\hline 14 & $1-21 x+98 x^{2}-49 x^{3}$ & 21 & 24 \\
\hline 16 & $1-24 x+148 x^{2}-176 x^{3}+4 x^{4}$ & 10 & 12 \\
\hline 8 & $1-20 x+16 x^{2}-16 x^{3}$ & 8 & 7 \\
8 & $1-26 x+242 x^{2}-960 x^{3}+1685 x^{4}-1138 x^{5}$ & 6 & 6 \\
\hline 10 & $1-24 x+128 x^{2}-289 x^{3}$ & - & 4 \\
10 & $1-46 x+866 x^{2}+\cdots-56642 x^{9}$ & - & 6 \\
\hline
\end{tabular}

As for the high temperature analysis we have confirmation that some of the singularities of the linear ODE of $\Phi_{H}^{(n)}$ are actually singularities of the linear ODE of the $\tilde{\chi}^{(n)}$. All the Nickelian singularities for $n=8,10,12$ are confirmed. Of the five (nine) Nickelian singularity polynomials for $n=14(n=16)$ four (eight) are missing.

As for the new singularities, $1-2 w=0$ for the ODE of $\tilde{\chi}^{(5)}$ and $1-8 w^{2}=0$ for the ODE of $\tilde{\chi}^{(6)}$, we should say that these singularities occur for higher index $n$. Thus, as far as $\tilde{\chi}^{(5)}$ and $\tilde{\chi}^{(6)}$ are concerned, and in view of the "limited" analysis carried out for $\tilde{\chi}^{(n)}, n>6$, there is no new singularity discovered that is not in the "known" 
set (given by the Nickelian singularities and the singularities of the $\Phi_{H}^{(n)}$ integrals, irrespective of the index $n>6$ ).

\subsection{Local exponents}

We turn now to the indicial exponents at some of the singularities found in the analysis of (43) and (44).

Here, the situation may seem different from the equivalent analysis made for $\tilde{\chi}^{(5)}$ and $\tilde{\chi}^{(6)}$. For instance, when we consider the indicial exponents for the singularities that should occur in the still unknown linear ODE for, e.g. $\tilde{\chi}^{(8)}$, the series that we analyse contain contributions from all the even $\tilde{\chi}^{(n)}$ up to $\sqrt{N}$. For instance, for $(1-4 x)=0$, this singularity is also a singularity of the linear ODE of $\tilde{\chi}^{(10)}, \tilde{\chi}^{(12)}$, etc. One may thus expect to obtain for the Nickelian singularity $(1-4 x)=0$ the local exponent [4] $\left(n^{2}-3\right) / 2$, for $n=8,10$ and $n=12$. The differences between all three exponents being integers, it is the value $61 / 2$ corresponding to $n=8$ which should appear. What we have obtained is indeed that for the Nickelian singularities, when the exponents have stabilized, they agree with $\left(n^{2}-3\right) / 2$, where $n$ is the lowest index.

Here we give some other examples of local exponents. For the non-Nickelian singularity polynomial $\left(1-26 x+242 x^{2}-960 x^{3}+1685 x^{4}-1138 x^{5}\right)$ corresponding to $n=8$, the local exponent is 23.5 (with 4 digits for $q=14,5$ digits for $q=17$ and 3 digits for $q=20)$. For the non-Nickelian singularity polynomial $\left(1-20 x+16 x^{2}-16 x^{3}\right)$ corresponding to $n=8$, the local exponent is 18.5 (with 2 digits for $q=14,3$ digits for $q=17$ and 2 digits for $q=20$ ). Both exponents agree with (E.1) with $n=8$ and respectively $m=1$ and $m=2$.

Similarly for the high temperature analysis, and for instance, for the Nickelian singularities given by roots of the polynomials $P_{1}, P_{2}$ and $P_{3}$ corresponding to $\tilde{\chi}^{(7)}$, the indicial exponent 23 appears with 10 correct digits using 480 terms at order 25 . The exponents for the non-Nickelian singularity polynomial $\left(1+12 w+54 w^{2}+112 w^{3}+\right.$ $\left.105 w^{4}+35 w^{5}+4 w^{6}\right)$, which should correspond to $\tilde{\chi}^{(7)}$, shows up as 17 with 2 correct digits.

Let us close this analysis with the following remark: the diff-Padé analysis of the sum (43) has shown the singularity $w=-1 / 2$ which was attributed to $\tilde{\chi}^{(9)}$. Actually $w=-1 / 2$ is known to be a Nickelian singularity for $\tilde{\chi}^{(9)}$. From the analysis of the $\Phi_{H}^{(n)}$ we found that $w=-1 / 2$ is also a singularity of $\Phi_{H}^{(7)}$. So this singularity may well be attributable to $\tilde{\chi}^{(7)}$. The diff-Padé analysis gives for this singularity 39 as the local exponent, which is the value predicted by $\left(n^{2}-3\right) / 2$ for $\tilde{\chi}^{(9)} \S$.

\section{4. "Indicial" exponents of non-holonomic sums}

We showed in previous subsections that even if the series of $\tilde{\chi}^{(7)}$ is "polluted" by the terms of the other $\tilde{\chi}^{(n)}$ the diff-Pade analysis is efficient enough to give the correct singularities and the corresponding indicial exponents.

Recall that the sums $(43,44)$ are believed to be non-holonomic, thus our linear ODE search (diff-Padé analysis) is just an approximation and another way to encode, via the singularities and their local exponents, the information contained in the series coefficients.

$\S$ We may thus conclude that either the linear ODE of $\tilde{\chi}^{(7)}$ does not have this singularity or it has this singularity, but the exponent is compatible with 39 , i.e. it may be $(\rho)^{p}$ with $\rho \geq 39, p \geq 1$. 
For this series with infinitely many singularities, generically not suitable for a diff-Padé analysis, it is interesting to see how the local exponents actually appear for those singularities occurring in all $\tilde{\chi}^{(n)}$, i.e. $w= \pm 1 / 4$.

To more fully appreciate the results of this section, consider the "holonomic" sum $\tilde{\chi}^{(1)}+\tilde{\chi}^{(3)}+\tilde{\chi}^{(5)}$. One may ask what are the local exponents that appear for $w=1 / 4$, knowing the exponents for each term? Recall that at $w=1 / 4$, the local exponent for the linear ODE of $\tilde{\chi}^{(1)}$ is -1 , for $\tilde{\chi}^{(3)}$ they are $-3 / 2,(-1)^{2}$ and $(0)^{2}$ and for the linear ODE of $\tilde{\chi}^{(5)}$, (from diff-Padé) the observed exponents are $-3 / 2,-1$ and $(0)^{4}$.

A diff-Padé analysis on the holonomic sum $\tilde{\chi}^{(1)}+\tilde{\chi}^{(3)}+\tilde{\chi}^{(5)}$ should show all these exponents (with possibly a change in the multiplicity due to auto-cancellation) and possibly other exponents that differ by integer values from the exponents in each individual $\tilde{\chi}^{(n)}$ term. This last possibility comes from a cancellation between the initial terms of the series.

Putting the known singularities into the head polynomial of an order fifteen linear ODE and using 860 terms we obtain:

$$
w=1 / 4, \quad-3 / 2,-1,0^{3}, 1 .
$$

Thus we see that here is no new exponent not differing by integer values from the exponents of each individual $\tilde{\chi}^{(n)}$ term.

Let us now return to considering the non-holonomic infinite sum, $\tilde{\chi}_{H}=\tilde{\chi}^{(1)}+$ $\tilde{\chi}^{(3)}+\cdots$, for which a diff-Padé analysis (with some 2000 terms and orders 15 and 18 ) gives, at $w=1 / 4$, the local exponents:

$$
w=1 / 4, \quad-1,-1 / 8,(3 / 8)^{2},(15 / 8)^{2},(35 / 8)^{2}
$$

Here we see the generation of new exponents that can only be seen as a consequence of the non-holonomic character of the sum. Note that the new exponents are still rational numbers. Note also that the new exponents appear not only in a diff-Padé analysis of $\tilde{\chi}_{H}$, but also in $\tilde{\chi}_{H}-\tilde{\chi}^{(1)}-\tilde{\chi}^{(3)}$ or in $\tilde{\chi}_{H}-\tilde{\chi}^{(1)}-\tilde{\chi}^{(3)}-\tilde{\chi}^{(5)}$. One has some kind of "self-similarity" in $\tilde{\chi}$ as far as the indicial exponents are concerned. Note that the multiplicities above may increase with more terms.

The local exponents are those of the full $\tilde{\chi}_{H}$ around $w=1 / 4$. They can be checked in

$$
\frac{\left(1-s^{4}\right)^{1 / 4}}{s} \tilde{\chi}_{H}=\text { const } \cdot \tau^{-7 / 4} F_{+}+B_{f}
$$

given by Orrick et al., using $F_{+}$and $B_{f}$ in the Appendix in [8], by switching from the variable $\tau$ to the variable $(w-1 / 4)$.

The following section presents a simple model showing the mechanism by which a resummation of the infinite number of logarithmic singularities proliferating in the holonomic $n$-fold integrals $\tilde{\chi}^{(n)}$ can lead to the known power-law singularities in $\chi$.

\section{Convergence of the $n$-particle sequence}

It was already observed by $\mathrm{Wu}$ et al. [2] that for the leading divergence of the susceptibility proportional to $|\tau|^{-7 / 4}$ (where $\tau=(1 / s-s) / 2$ ), the sequence of partial sums of either $\chi^{(2 n)}$ or $\chi^{(2 n+1)}$ appears to converge exceptionally rapidly to the corresponding susceptibility below or above the critical temperature. This has been confirmed numerically to higher order by Bailey et al. [25], who estimated that, asymptotically, the amplitudes $I_{n}$ of the leading divergence of $\chi^{(n)}$ are in the ratio $I_{n+2} / I_{n}<1 / 1000$. On the other hand, the leading correction terms in $\chi^{(1)}$ and $\chi^{(2)}$ 
are easily seen to be of order $|\tau|^{1 / 4}$ and $|\tau|^{1 / 4} \log |\tau|$ respectively whereas the leading correction in $\chi$ approaches a constant in the limit $\tau \rightarrow 0$. In fact the exact solutions for $\chi^{(3)}$ and $\chi^{(4)}$, found by Zenine et al. $[10,12]$ make it plausible that every partial sum of $\chi^{(2 n)}$ or $\chi^{(2 n+1)}$ will, asymptotically in the limit $\tau \rightarrow 0$, give a vanishingly small contribution to the leading correction term. This raises the issue of the nature of the convergence of the $n$-particle sequence to the susceptibility.

With the exceptionally long series now available for $\chi^{(5)}$ and $\chi^{(6)}$ we can, even in the absence of an exact linear ODE solution, make numerically precise estimates of the correction terms in these functions. A scheme that works well is a combination of unwanted singularity suppression as described in [3] and function fitting. The latter is done by assuming various combinations of powers and powers of logarithms with unknown coefficients and generating the corresponding series in $s$. The unknown coefficients can then be estimated by least squares fitting to the highest order terms of the exact (unwanted singularity suppressed) series. This fitting is typically done iteratively with the leading order exact coefficient values substituted for the numerically estimated ones from an earlier iteration.

Then, by combining this information with what is already known for the lower orders $\chi^{(n)}$, we can build a plausible "toy model" $\chi_{\text {Toy }}^{(n)}$ that can be easily extrapolated to $n \rightarrow \infty$. This gives us, at least qualitatively and even semi-quantitatively, a picture of the convergence of the partial sums of $\chi^{(n)}$ to the full $\chi$. The details of this extrapolation procedure via $\chi_{\text {Toy }}^{(n)}$ is described below.

\subsection{Behaviour of $\chi^{(n)}$}

We begin by providing a summary of the behaviour of $\chi^{(n+1)}$ near the ferromagnetic critical point.

The normalization factor included for convenience on the left-hand side of the equations below is $4 \pi^{n} n$ ! while the factor $\sqrt{s}$ is crucial to give series increasing in even powers of $\tau$ only. An empirical observation is that $\tau=(1 / s-s) / 2$ appears almost universally in all formulae in the combination $|\tau| / 4$ so we have defined $\tau_{4}=|\tau| / 4$. The known $\chi^{(n)}$ terms are $\|$

$$
\begin{aligned}
& 4 \sqrt{s} \cdot \chi^{(1)}= 4 \cdot\left(\frac{1}{8} \tau_{4}^{-7 / 4}+\tau_{4}^{1 / 4}\right)-\tau_{4}^{1 / 4}+\mathrm{O}\left(\tau_{4}^{9 / 4}\right) \\
& 4 \pi \sqrt{s} \cdot \chi^{(2)}= \frac{1}{3} \cdot\left(\frac{1}{8} \tau_{4}^{-7 / 4}+\tau_{4}^{1 / 4}\right)+\left(\log \tau_{4}+\frac{11}{12}\right) \cdot \tau_{4}^{1 / 4}+\mathrm{O}\left(\tau_{4}^{9 / 4}\right) \\
& 8 \pi^{2} \sqrt{s} \cdot \chi^{(3)}=8 I_{3}^{+} \pi^{2} \cdot\left(\frac{1}{8} \tau_{4}^{-7 / 4}+\tau_{4}^{1 / 4}\right) \\
&-\left(\log ^{2} \tau_{4}+\frac{23}{6} \log \tau_{4}+\frac{\pi^{2}}{3}+\frac{41}{36}\right) \cdot \tau_{4}^{1 / 4}+\mathrm{O}\left(\tau_{4}^{9 / 4-\epsilon}\right) \\
& 24 \pi^{3} \sqrt{s} \cdot \chi^{(4)}=24 I_{4}^{-} \pi^{3} \cdot\left(\frac{1}{8} \tau_{4}^{-7 / 4}+\tau_{4}^{1 / 4}\right)+\left(\log ^{3} \tau_{4}+\frac{35}{4} \log ^{2} \tau_{4}\right. \\
&\left.+\left(2 \pi^{2}+\frac{107}{12}\right) \log \tau_{4}+34.3411462895 \ldots\right) \cdot \tau_{4}^{1 / 4}+\mathrm{O}\left(\tau_{4}^{9 / 4-\epsilon}\right), \\
& 96 \pi^{4} \sqrt{s} \cdot \chi^{(5)}=96 I_{5}^{+} \pi^{4} \cdot\left(\frac{1}{8} \tau_{4}^{-7 / 4}+\tau_{4}^{1 / 4}\right)-\left(\log ^{4} \tau_{4}+\frac{47}{3} \log ^{3} \tau_{4}\right.
\end{aligned}
$$

\| The formula given here for $\chi^{(4)}$ corrects misprints in [15]. 


$$
\begin{aligned}
+ & \left(6 \pi^{2}+\frac{245}{6}\right) \cdot \log ^{2} \tau_{4}+305.6550541085 \ldots \log \tau_{4} \\
& +375.271992213596336733341995793) \tau_{4}^{1 / 4}+\mathrm{O}\left(\tau_{4}^{9 / 4-\epsilon}\right), \\
480 \pi^{5} \sqrt{s} \cdot \chi^{(6)}= & 480 I_{6}^{-} \pi^{5} \cdot\left(\frac{1}{8} \tau_{4}^{-7 / 4}+\tau_{4}^{1 / 4}\right)+\left(\log ^{5} \tau_{4}+\frac{295}{12} \log ^{4} \tau_{4}\right. \\
+ & \left(\frac{40 \pi^{2}}{3}+\frac{2275}{18}\right) \cdot \log ^{3} \tau_{4}+1437.2558956691 \ldots \ln ^{2} \tau_{4} \\
+ & 4238.8509988858869410798745 \log \tau_{4} \\
& +5284.244417602341195112209) \cdot \tau_{4}^{1 / 4}+\mathrm{O}\left(\tau_{4}^{9 / 4-\epsilon}\right)
\end{aligned}
$$

while the complete summation of all $\chi^{(n)}$ yields the exact

$$
\begin{aligned}
\sqrt{s} \cdot \chi & =1.00081526044021 \ldots\left(\frac{1}{8} \tau_{4}^{-7 / 4}+\tau_{4}^{1 / 4}\right)-0.1041332450938 \ldots \\
& +(0.0323522684773 \ldots \log (\tau)-0.074368869753 \ldots) \cdot \tau+\mathrm{O}\left(\tau^{2}\right)
\end{aligned}
$$

for $\tau>0$ and

$$
\begin{aligned}
\sqrt{s} \cdot \chi & =\frac{1.00096032872526 \ldots}{12 \pi} \cdot\left(\frac{1}{8} \tau_{4}^{-7 / 4}+\tau_{4}^{1 / 4}\right)-0.1041332450938 \ldots \\
& +(0.0323522684773 \ldots \log (-\tau)-0.074368869753 \ldots) \cdot \tau+\mathrm{O}\left(\tau^{2}\right)
\end{aligned}
$$

for $\tau<0$. We have used $\mathrm{O}\left(\tau_{4}^{9 / 4-\epsilon}\right)$ to indicate $\mathrm{O}\left(\tau_{4}^{9 / 4}\right)$ with logarithmic corrections. We also distinguish between numerical constants. Those with trailing ... are known to much higher accuracy but have not yet been recognized in terms of elementary constantst. The others are believed, but not guaranteed, to be accurate to the number of digits given.

An important remark about the form of equations (48) is that we have split the $\tau_{4}^{1 / 4}$ contribution to create the combination $\left(\frac{1}{8} \tau_{4}^{-7 / 4}+\tau_{4}^{1 / 4}\right)$ which is the scaling part of the full susceptibility in (50). The original motivation for this was the observation by Orrick et al. [8] that the terms in the expansion of the scaling function in the susceptibility are $\tau_{4}^{-7 / 4+2 n}$ without logarithms, while in the expansion of the background part of the susceptibility there are only integer powers of $\tau_{4}$ but now with logarithmic corrections. A simple way to reproduce the scaling part of the susceptibility from the $\chi^{(n)}$ sum is to replace every $\tau_{4}^{-7 / 4}$ by the scaling function, as we have done. Then the sum rule on the $I_{n}$ that ensures the correct $\tau_{4}^{-7 / 4}$ amplitude in the susceptibility will also automatically yield the correct scaling function. Such separation into "scaling" and "background" in individual $\chi^{(n)}$ would appear to be completely arbitrary except that we now find that the "background" remainder in $\chi^{(n)}$ has a simple dependence on $n$ in which the same formulae apply simultaneously to both odd and even $n$.

This was quite unexpected and the separation may well only apply to the leading correction term we are concerned with here. As a practical matter, it leads us directly

$\dagger$ The exact values for $I_{3}^{+}$and $I_{4}^{-}$have been given by Tracy [27] (see also note added in proof in [15]). Highly accurate numerical values for $I_{5}^{+}$and $I_{6}^{-}$are given by Bailey et al. [25]. For the constants in $\chi$ see Orrick et al. [8]. The constant of $\chi^{(4)}$ in (48), to higher accuracy is 34.3411462895318287823760337015134958201194891883303683732355064700755161069888189217774 $8221462488591136755297844305262613713337765 \cdots$ 
to the conjecture

$$
\begin{aligned}
4 n ! \pi^{n} & \sqrt{s} \cdot \chi^{(n+1)}=4 n ! \pi^{n} I_{n+1} \cdot\left(\frac{1}{8} \tau_{4}^{-7 / 4}+\tau_{4}^{1 / 4}\right) \\
& -(-1)^{n}\left[\log ^{n} \tau_{4}+n\left(n-\frac{1}{12}\right) \cdot \log ^{n-1} \tau_{4}\right. \\
& +\left(\begin{array}{c}
n \\
2
\end{array}\right)\left(n^{2}+\frac{\left(2 \pi^{2}-19\right) n}{6}+\frac{125-12 \pi^{2}}{36}\right) \cdot \log ^{n-2} \tau_{4} \\
& +\left(\begin{array}{c}
n \\
3
\end{array}\right)\left(n^{3}+\frac{\left(4 \pi^{2}-37\right) n^{2}}{4}+\frac{\left(374-37 \pi^{2}\right) n}{12}-0.441452610 \ldots\right) \cdot \log ^{n-3} \tau_{4} \\
\ldots & \left.+\left(\begin{array}{l}
n \\
k
\end{array}\right)\left(n^{k}+k\left(k\left(\pi^{2}-9\right)+\frac{17}{2}-\pi^{2}\right) \frac{n^{k-1}}{6}+\ldots\right) \cdot \log ^{n-k} \tau_{4}+\ldots\right] \cdot \tau_{4}^{1 / 4} \\
& +\mathrm{O}\left(\tau^{9 / 4-\epsilon}\right)
\end{aligned}
$$

based on the known low order results and applicable equally to the logarithmic and non-logarithmic terms. Note that the numerical constant in (51) is simply related to the constant in $\chi^{(4)}$ deduced from its linear ODE solution. Specifically, one has $-0.44145 \ldots=34.341 \ldots+\pi^{2} / 4-149 / 4$.

\subsection{Resummation of the toy model}

The conjectured general term (51) forms the basis for our $\chi_{\text {Toy }}^{(n+1)}$. The leading divergence in $\chi^{(n+1)}$, now combined with the scaling function, is not under consideration here since we have nothing to add to what is already known. Of the correction terms in (51) we will capture exactly the leading $\log ^{n} \tau_{4}$ into our toy model. The appearance of binomial coefficients in the next three terms is suggestive of a formula like $\left(\log \tau_{4}+n\right)^{n}$ which would capture the leading $n$ dependence correctly. We do not have enough information to be confident about the behaviour of any lower order logarithmic terms and so at this point our model becomes dictated by the criteria of simplicity. The numerical values of the lower order terms suggest the simple formula $\left(\log \tau_{4}+\lambda n\right)^{n}$ as reasonable, where $\lambda \approx 1$ is some as yet undetermined constant. If $\lambda$ is very close to 1.0 then our constant term $(\lambda n)^{n}$ is probably an underestimate but it is worth remarking that in the $\tau \rightarrow 0 \operatorname{limit}, \log \tau_{4}$ is negative. This implies successive terms in the expansion of $\left(\log \tau_{4}+\lambda n\right)^{n}$ alternate in sign and the final value, just as in the exact $\chi^{(n+1)}$, is the result of large cancellations between terms. Thus it is more important that we capture correctly the "smoothness" by which successive terms vary and this is hard to estimate from the limited data available. Let these caveats be understood. Let us denote by $\Delta$ the correction to scaling. Then our toy model for the correction to scaling terms is

$$
\begin{aligned}
& \sqrt{s} \cdot \Delta \chi_{\text {Toy }}^{(n+1)}=-\frac{\tau_{4}^{1 / 4}}{4 \pi^{n} n !} \cdot\left(\log \left(1 / \tau_{4}\right)-\lambda n\right)^{n}, \\
& \sqrt{s} \cdot \Delta \chi_{\text {Toy }}=\sum \sqrt{s} \cdot \Delta \chi_{\text {Toy }}^{(n)}
\end{aligned}
$$

with the sum understood to be over even $n$ for $T<T_{c}$ and odd $n$ for $T>T_{c}$. As $\tau \rightarrow 0$, the sum in (52) is dominated by large $n$ and can be replaced by an integral that is easily treated by steepest descent methods. The value of the integral will depend on the undetermined $\lambda$ and we will choose $\lambda$ such that, in the limit $\tau \rightarrow 0, \sqrt{s} \cdot \Delta \chi_{\text {Toy }} \rightarrow A_{\text {Toy }}$ is a constant. That this is possible is verified by explicit 
calculation below. The reasonableness of our toy model can then be judged by how close $A_{\text {Toy }}$ is to the exact $A=-0.1041 \ldots$ from (50).

The dominant $n$ dependence of the integrand in the integral approximation for $\sqrt{s} \cdot \Delta \chi_{\text {Toy }}$ is the factor $\exp \left[n \log \left(\log \left(1 / \tau_{4}\right)-\lambda n\right)-n \log (n \pi)+n\right]$ and this has a maximum at $n_{p}$ where:

$$
n_{p}=\frac{p}{1+p \lambda} \cdot \log \left(1 / \tau_{4}\right), \quad p \cdot \lambda+\log (\pi p)=0 .
$$

The exponential at its maximum evaluates to $\exp \left(p \log \left(1 / \tau_{4}\right)\right)=1 / \tau_{4}^{p}$ which establishes the dominant $\tau$ dependence of the integral as a function of $\lambda$ since $p=p(\lambda)$ is the solution of the transcendental equation in (53). Expanding the exponential about its maximum then gives the approximate $\sqrt{s} \cdot \Delta \chi_{\text {Toy }}^{(n+1)}$ and the required sum in (52), namely

$$
\sqrt{s} \cdot \Delta \chi_{\text {Toy }} \approx \frac{-\tau_{4}^{1 / 4-p}}{8 \sqrt{2 \pi n_{p}}} \int d n \exp \left[-(1+p \lambda)^{2}\left(n-n_{p}\right)^{2} /\left(2 n_{p}\right)\right]=-\frac{\tau_{4}^{1 / 4-p}}{8(1+p \lambda)}
$$

valid in the limit $\tau \rightarrow 0$ both above and below $T_{c}$. The choice $p=1 / 4$ is now seen as necessary and we get from (53) and (54)

$$
\begin{aligned}
& \lambda=4 \log (4 / \pi) \approx 0.966 \\
& A_{\text {Toy }}=\lim _{\tau \rightarrow 0} \sqrt{s} \cdot \Delta \chi_{\text {Toy }}=-\frac{1}{(8+8 \log (4 / \pi))} \approx-0.1007
\end{aligned}
$$

The close agreement of the asymptotic amplitude with the exact $A \approx-0.1041$ gives us confidence that (52), with the specific choice of $\lambda$ from (55), will be usefully predictive for finite $\tau$ and $n$.

The result of numerical computation for finite $\tau$ and $n$ is shown for $T<T_{c}$ in Fig. 1. A similar plot could be made for $T>T_{c}$. The agreement between exact and toy partial sums at low order is of course by design and the agreement in the limit $\tau \rightarrow 0$ has already been remarked on in connection with (55). The striking feature of the shifts in $\log _{10}(-\tau)$ with order can be deduced from (53). The $n_{p}$ in (53) which corresponds to the $n$ of maximal contribution is also the $n$ characterising the transition region of the partial sum approximations to $\sqrt{s} \cdot \Delta \chi_{\text {Toy }}$ between the asymptotes 0 and $A_{\text {Toy }}=-0.1007$. Then, since each additional order is a change in $n$ of 2 , we deduce a transition region shift of $\Delta \log \left(1 / \tau_{4}\right)=8 \cdot(1+\log (4 / \pi)) \approx 10$, or about four decades in temperature.

\section{Natural boundary: power spectrum analysis}

In this section we are using the power spectrum analysis introduced in Sec 6.3 of Orrick et al. [8], though not fully described there. The model of $n$-fold integrals [14] has shown that the singularities of the linear ODE of $\Phi_{H}^{(n)}$ occur in the linear ODE

of the higher order $\Phi_{H}^{(n+2 m)}$. These model integrals are assumed to "mimic" correctly the $\chi^{(n)}$ in terms of the locus of the singularities. With the singularities obtained here from diff-Padé analysis of $\tilde{\chi}^{(5)}$ and $\tilde{\chi}^{(6)}$ we have confirmation of this feature. If we focus on the singularities which are on the unit circle $|s|=1$, the Nickelian singularities of $\tilde{\chi}^{(3)}$, (i.e. $\left.(1-w)(1+2 w)=0\right)$ are also singularities of the linear ODE of $\tilde{\chi}^{(5)}$.

However, the detailed Landau conditions analysis (see Appendix B) proves that the above singularities lying on $|s|=1$ do not occur on the principal disc of $\tilde{\chi}^{(5)}$. 


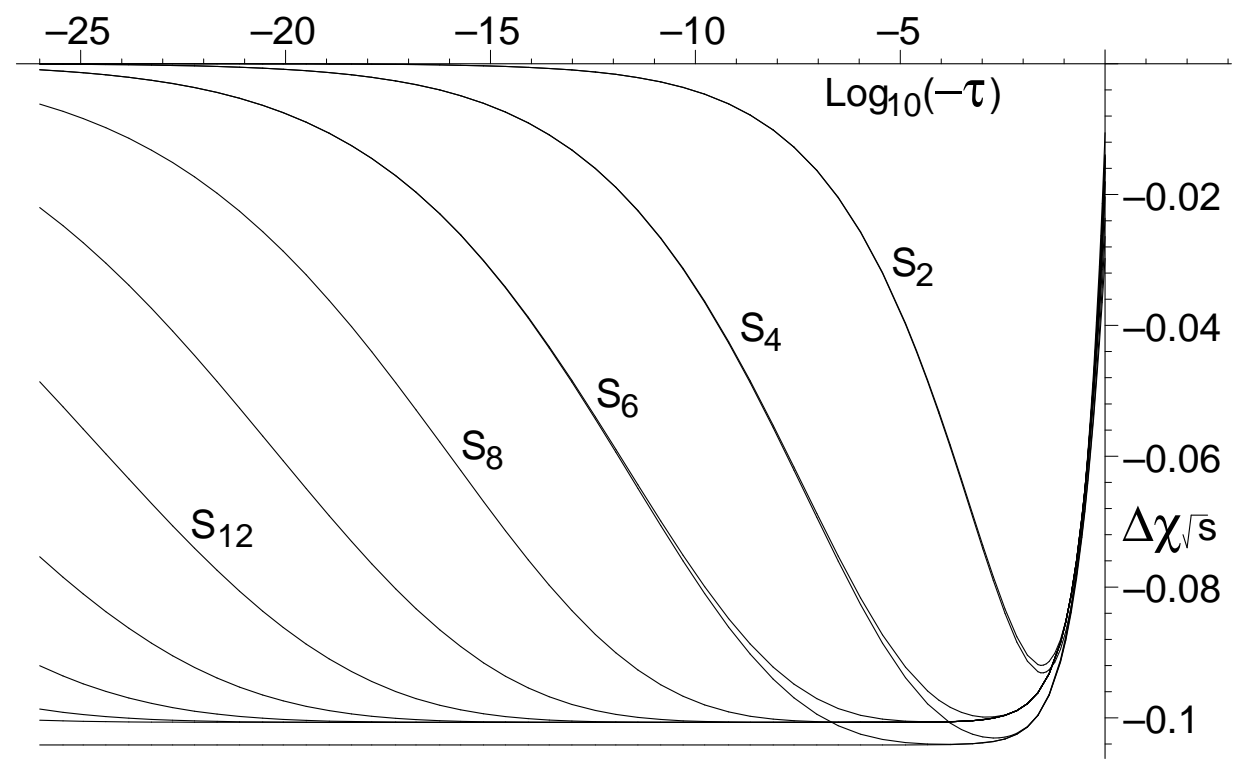

Figure 1. The contribution to the "background" part of the susceptibility from the partial sums $S_{2}=\chi^{(2)} \sqrt{s}, S_{4}=S_{2}+\chi^{(4)} \sqrt{s}, S_{6}=S_{4}+\chi^{(6)} \sqrt{s}$ and the exact $\chi \sqrt{s}$ based on the formulae (1) and (2) exclusive of the scaling $\frac{1}{8} \tau_{4}^{-7 / 4}+\tau_{4}^{1 / 4}$ combination. Also shown are the corresponding toy model $S_{n}$ based on (52) now extending to larger $n$.

Indeed, this is just a special case of the general theorem that applies to all the Case 3-5 singularities. Furthermore, the analysis in Appendix D suggests that the singularities associated with the ODE head polynomial factors $(1-2 w)$ for $\tilde{\chi}^{(5)}$ and $\left(1-8 w^{2}\right)$ for $\tilde{\chi}^{(6)}$ are not even Landau singularities of the integrals $\tilde{\chi}^{(5)}$ and $\tilde{\chi}^{(6)}$, at least in a Landau singularities analysis that does not fully take into account the Fermionic term.

Here we confirm the limited result of the absence of these singularities on $|s|=1$ on the principal disc using a technique [8] based on the fast-Fourier transform of the series of $\chi^{(n)}$. The series is first appropriately modified by removing the dominant singularities and smoothing.

The Nickelian singularities $s_{0}$ have very small amplitude, roughly $\left(1-s / s_{0}\right)^{n^{2} / 2}$ for $\tilde{\chi}^{(n)}$. For $\tilde{\chi}^{(5)}$ the effect on the series coefficients (for series in the $s$ variable) at $N=2000$ is roughly $1 /(2000)^{13}=10^{-43}$ times smaller than that from the ferromagnetic singularity. The amplitude of the dominant ferromagnetic divergence being known this contribution can be subtracted. As the smoothing continues (see details in [3]), the series coefficients will start to decay rapidly with order $N$ and this must be corrected for by multiplying, at each step, by some power of $N$ to again make the series coefficients roughly constant in $N$. Once the series have been smoothed and multiplied up by about $N^{13}$ the FFT is performed.

The FFT is obtained by using say 512 coefficients $a_{n+N}$ starting from some large $N$ to utilise the highest order coefficients available. We get

$$
b_{m}=\sum a_{n+N} \cdot \exp \left(2 \pi \mathrm{i} n \frac{m}{512}\right), \quad m=1,2, \ldots, 512 .
$$




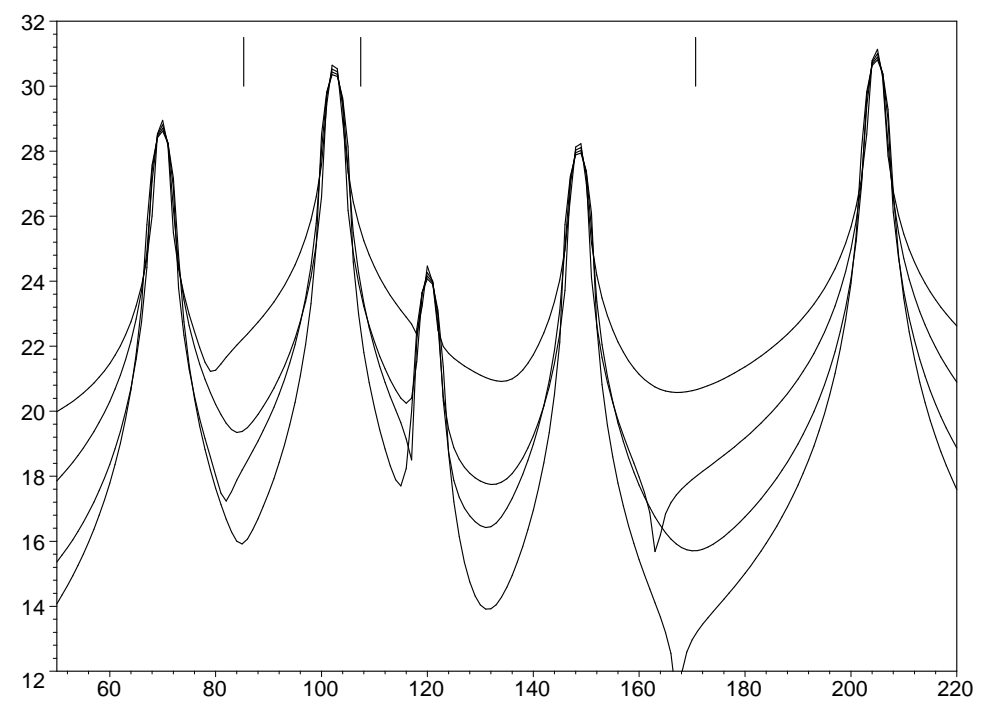

Figure 2. Power spectrum of $\chi^{(5)}$ series on $\log _{10}$ scale vs "frequency" $m$

and the power spectrum $P_{m}=b_{m} \cdot b_{512-m}$ which runs over 256 points. The "frequency", $m$, is directly interpretable as an angle $\theta=\pi m / 256$ giving the locations of the singularities $\exp ( \pm \mathrm{i} \theta)$ in the complex $s$ plane. Windowing $a_{n} \rightarrow$ $a_{n} \sin ^{p}(n \pi / 512)$ can be used to reduce background (this broadens the spectral lines).

The power spectrum of $\tilde{\chi}^{(5)}$ series is shown on Fig. 2. The curves correspond to various values of the integers $p=2, \cdots, 5$. From left to right, the first and third spikes correspond to the singularities of $1-3 w+w^{2}=0$. The second and fifth spikes correspond to $1+2 w-4 w^{2}=0$ and the singularity $w=-1$ appears as the fourth spike.

The singularities $w=1 / 2, w=1$ and $w=-1 / 2$ are not seen. Their positions are indicated, respectively, by the vertical lines at the top of the spectrum.

Similar analysis of the series of $\tilde{\chi}^{(6)}$ has shown the non-occurrence of the spike corresponding to the non-Nickelian singularity $w^{2}=1 / 8$ which is on $|s|=1$.

The FFT analysis does show, in all examples so far, that there is no evidence for any singularities on the $|s|=1$ boundary of the physical sheet other than the Nickelian singularities. Thus the possibility that there will be "destruction" of the natural boundary by cancellation of the singularities accumulating on the unit circle is becoming more and more remote.

\section{Conclusion}

We have calculated series of some 2000 or more terms for the magnetic susceptibility (high- and low-temperature regime of the isotropic Ising model) as well as for the individual five- and six-particle contributions $\tilde{\chi}^{(5)}$ and $\tilde{\chi}^{(6)}$. This was achieved by use of modular arithmetic which amounts to generating the coefficients modulo a set of primes, then using the Chinese remainder to obtain the exact coefficients.

By extending the series for $\tilde{\chi}^{(5)}$ modulo a single prime to 10000 terms we have discovered the exact linear ODE for $\tilde{\chi}^{(5)}$ modulo that prime. The Russian-doll 
structure previously found to occur for $\tilde{\chi}^{(3)}$ and $\tilde{\chi}^{(4)}$, and conjectured for the linear differential operators of the $\tilde{\chi}^{(n)}$, is actually confirmed for $\tilde{\chi}^{(5)}$, as well as a stronger direct sum structure.

We have described our present algorithm for finding the linear ODE satisfied by a series $S(x)$ in some detail because it is robust and particularly efficient. It is based on an ansatz of a rectangular array of coefficients of size fixed by degree $D$ in $x$ and order $M$ in $x \mathrm{~d} / \mathrm{d} x$. The use of the operator $x \mathrm{~d} / \mathrm{d} x$ rather than $\mathrm{d} / \mathrm{d} x$ guarantees that the resulting ODE is Fuchsian. Now suppose we have found the ODE with minimum $D$ for given $M$. If $N$ is the number of series terms required for this $D, M$ combination then an empirical observation is that there exists a linear relationship between $N, M$ and $D$ that enables us to infer the order $M_{0}$ of the minimum order ODE required by $S(x)$ without having to obtain the minimum order ODE itself or even having to obtain any singularity information whatsoever. We have no explanation for this linear relationship and leave it as a challenge for the reader to provide an analytic basis for our "magic formula" (30).

The exact long series have been used in diff-Padé analysis to obtain the singularities that should occur in the linear ODE of $\tilde{\chi}^{(5)}$ and $\tilde{\chi}^{(6)}$. We have confirmation that the singularities of the ODEs of the $n$-fold integrals $\Phi_{H}^{(5)}$ and $\Phi_{H}^{(6)}$ (which differ from $\tilde{\chi}^{(5)}$ and $\tilde{\chi}^{(6)}$ by the absence of the Fermionic factor) are all singularities of the ODEs of $\tilde{\chi}^{(5)}$ and $\tilde{\chi}^{(6)}$. However, our diff-Padé analysis showed that these linear ODEs have additional singularities, namely $w=1 / 2$ for the linear $O D E$ of $\tilde{\chi}^{(5)}$ and $w^{2}=1 / 8$ for the $O D E$ of $\tilde{\chi}^{(6)}$, not predicted by the corresponding $\Phi_{H}^{(n)}$. We see that the Fermionic factor does not affect the singularities corresponding to $\Phi_{H}^{(n)}$, but it does bring additional singularities to the linear ODE. From a Landau singularity analysis viewpoint this is not a surprise: A new factor cannot move a singularity, but it can either give rise to cancellations or introduce new singularities, and we have seen that cancellation does not occur. Our exact mod prime ODE for $\tilde{\chi}^{(5)}$ confirms the accuracy of the diff-Padé results and in particular proves that the ODE for $\tilde{\chi}^{(5)}$ carries the extra singularity at $w=1 / 2$.

We do not know whether or not these extra singularities of the $\tilde{\chi}^{(n)}$ ODEs are singularities of the integrals (5). The most common case is that in which all the singularities of the ODE and the integral are the same; this is the situation for $\tilde{\chi}^{(3)}$ if one takes into account its various analytical continuations (cf. first footnote in Appendix B). As a specific example, both the Landau analysis and the ODE for $\tilde{\chi}^{(3)}$ predict a singularity at $s=(-1+\mathrm{i} \sqrt{7}) / 4$ for which it is to be noted $|s|<1$. While $\tilde{\chi}^{(3)}$ on the principal disc is not singular at this point there exists an analytic continuation of $\tilde{\chi}^{(3)}$ that isł. Our analysis of toy integrals in Appendix D provides an example of a difference between the singularities of the ODE and those of the integral. The ODE for the toy analog of $\tilde{\chi}^{(5)}$ has singularities at $w=1 / 2$ and $w^{2}=1 / 8$ but our Landau analysis of the toy integral fails to find singularities at these points. This may be a genuine distinction, or it may be that we missed something in the Landau analysis, or that the Landau analysis can't be guaranteed to give all singularities. It would be extremely useful to have other (simpler) examples where it can be shown that the ODE defined by an integral has more singularities than the integral.

A further check on the occurrence of the singularities (of the linear ODE's of $\Phi_{H}^{(n)}$ ) has been made for some $\tilde{\chi}^{(n)}, n \geq 7$, by subtracting, from the full $\tilde{\chi}$, the

$\ddagger$ This can be inferred from the connection matrices provided in [15]. 
known $\tilde{\chi}^{(1)}, \tilde{\chi}^{(3)}$ and the now long $\tilde{\chi}^{(5)}$ series. The diff-Padé analysis again confirms a large number of singularities occurring in the linear ODE's of the $\Phi_{H}^{(n)}$ that can, thus, be attributed to the corresponding linear $O D E$ of $\tilde{\chi}^{(n)}$. A similar analysis has been performed for the low-temperature regime.

While these diff-Padé analyses only yield approximate linear ODE's, they are efficient enough to give the indicial exponents at each singularity with good accuracy in most cases. An example of failure is that in the ODE for $\tilde{\chi}^{(5)}$ at $w=1 / 4$ where we detected $-3 / 2$ but not $-2,-7 / 4$ and $-5 / 4$, exponents also known to be present from our exact mod prime analysis. Thus while in all cases we have observed that indicial exponents are rational numbers, in the absence of this example we might have erroneously concluded that indicial exponents are either integer or half-integer. As a particularly striking success we obtained for $\tilde{\chi}^{(6)}$ at $w= \pm 1 / 4$ the indicial exponents 0 with a multiplicity of five $\left(0^{5}\right)$. While the diff-Padé analysis can only confirm this multiplicity of five as a lower bound it is the correct one at $w=1 / 4$ as shown by our exact (conjectured) equation (51).

From the linear combination of the long series for $\tilde{\chi}^{(5)}, \tilde{\chi}^{(6)}$ and $\tilde{\chi}$, we were able to make some serious progress on two important questions for a deeper physical and mathematical understanding of the full susceptibility $\tilde{\chi}$.

Firstly, we have, finally, resolved the issue of the power/log behaviour of each $\chi^{(n)}$ at the singular points versus the behaviour of the full $\chi$. The diff-Padé analysis performed for the full $\tilde{\chi}$ shows the non-occurrence, at $w= \pm 1 / 4$, of the logarithmic singularities corresponding to the individual $\tilde{\chi}^{(n)}$, but shows with good accuracy the indicial exponents known to occur in $\tilde{\chi}$. We presented a model showing the mechanism of the resummation of the infinite number of logarithmic singularities that proliferate in the holonomic $n$-fold integrals $\tilde{\chi}^{(n)}$ building the known divergence of $\tilde{\chi}$ at scaling.

Secondly, we have proved by a Landau analysis that no non-Nickelian singularities of $\Phi_{H}^{(n)}$ can lie on the $|s|=1$ boundary of the principal disc. Since we do not understand the origin of the additional ODE singularities at $w=1 / 2$ for $\tilde{\chi}^{(5)}$ and $w^{2}=1 / 8$ for $\tilde{\chi}^{(6)}$ we cannot make the same analytic claim. Instead we have verified this numerically by fast-Fourier transform on appropriately modified series. The FFT results are surprisingly clear-cut, and display peaks at the precise points of Nickelian singularities on the unit circle. The possibility of cancellation becomes more and more remote, up to a point where we are able to confirm the existence of a natural boundary for the full susceptibility.

In conclusion, we can say that, with this kind of experimental mathematics based on "extreme" massive computer calculations, we are exploring a new kind of "modular" lattice statistical mechanics, getting results that were unthinkable before. Indeed, we are now far along the road to a complete synthesis and understanding of the Ising model susceptibility. However, these experimental mathematical ideas for studying Ising model integrals are much more widely applicable, as they can be applied to any $n$-fold integral corresponding to a Feynman diagram. In such cases we are likely to be restricted only to the experimental aspects, leaving open the challenge for a subsequent theoretical understanding, such as that which we have been able to provide in the case of the Ising model. 


\section{Acknowledgments}

We would like to thank B. McCoy for many illuminating comments. We would like to thank A. Bostan for helping to find the $\tilde{\chi}^{(5)}$ ODE, modulo a prime, with his MAGMA program. We thank J. Dethridge for some optimization of earlier $\mathrm{C}++$ programs. The calculations presented in this paper would not have been possible without a generous grant of computer time on the server cluster of the Australian Partnership for Advanced Computing (APAC). We also gratefully acknowledge use of the computational resources of the Victorian Partnership for Advanced Computing (VPAC). One of us JMM would like to thank MASCOS, Melbourne for hospitality where part of this work was initiated and completed. This work is partially supported by a PICS/CRNS grant. This work has been performed without any ANR or ERC support. IJ and AJG gratefully acknowledge financial support from the Australian Research Council, and the hospitality of LPTMC, Université de Paris 6 where much of this work was carried out.

\section{E-mail or WWW retrieval of series}

The series for the various generating functions studied in this paper can be obtained via e-mail by sending a request to I.Jensen@ms.unimelb.edu.au or via the world wide web on the URL http://www.ms.unimelb.edu.au/ ${ }^{\sim}$ iwan/ by following the instructions.

\section{Appendix A. Singularity exponent sum-rules (Fuchs' relations)}

Suppose the order $M$ and degree $D$ linear ODE, $L_{M D}(S(x))=0$, with $L_{M D}$ given by (26), has singularities at $x=x_{i}$ with multiplicities $q_{i}$. Besides the true singularities of the generating function $S(x)$, the $x=x_{i}$ may be apparent singularities when $M=M_{0}$, the minimum order, or they may be $M$ dependent spurious singularities when $M>M_{0}$. In any case the ODE is necessarily of the form

$$
\begin{aligned}
& P_{D}(x) \cdot L_{x} \cdot\left(x \cdot \frac{\mathrm{d}}{\mathrm{d} x}\right)^{M-1}(S(x))+\cdots=0, \\
& L_{x}=x \cdot \frac{\mathrm{d}}{\mathrm{d} x}-s_{0}+\sum_{i}\left(q_{i} \cdot M-\frac{1}{2} q_{i}\left(q_{i}+1\right)-s_{i}\right) x /\left(x-x_{i}\right),
\end{aligned}
$$

with $x_{i}$ the roots of the degree $D$ head polynomial $P_{D}(x)$. One can explicitly verify that $s_{0}$ is the coefficient of $p^{M-1}$ in the indicial equation of degree $M$ at $x=0$,

$$
p^{M}-s_{0} p^{M-1}+\cdots+\text { constant }=0,
$$

and thus is the sum of $M$ exponents at $x=0$. Similarly, $s_{i}$ is the coefficient of $p^{q_{i}-1}$ in the indicial equation of degree $q_{i}$ at $x=x_{i}$,

$$
p^{q_{i}}-s_{i} \cdot p^{q_{i}-1}+\cdots+\text { constant }=0,
$$

and is the sum of the $q_{i}$ singularity exponents at $x=x_{i}$.

If we set $x=1 / y$, the transformed ODE (A.1) is

$$
\begin{aligned}
& P_{D}(1 / y) \cdot L_{y} \cdot\left(y \cdot \frac{\mathrm{d}}{\mathrm{d} y}\right)^{M-1}(S(1 / y))+\cdots=0, \\
& L_{y}=y \cdot \frac{\mathrm{d}}{\mathrm{d} y}+s_{0}-\sum_{i}\left(q_{i} \cdot M-\frac{1}{2} q_{i}\left(q_{i}+1\right)-s_{i}\right) /\left(1-y x_{i}\right),
\end{aligned}
$$


and we can identify $-s_{0}+\sum_{i}\left(q_{i} \cdot M-\frac{1}{2} q_{i}\left(q_{i}+1\right)-s_{i}\right)$ with $s_{\infty}$, the coefficient in of $p^{M-1}$ in the indicial equation of degree $M$ at $y=0$,

$$
p^{M}-s_{\infty} \cdot p^{M-1}+\cdots+\text { constant }=0
$$

and this coefficient is thus the sum of the $M$ exponents at $y=0$. This exponent equivalence is the sum-rule

$$
s_{0}+s_{\infty}+\sum_{i, \text { all }}\left(s_{i}+\frac{1}{2} q_{i}\left(q_{i}+1\right)\right)=M \cdot \sum_{i} q_{i}=M \cdot D,
$$

and is the starting point for our specialisations below. It is easy to show that (A.6) is equivalent to the usual Fuchs' relations $[28,29]$ which are sum-rules on all exponents. The advantage of (A.6) is that it makes explicit the role of those singularity exponents that arise as solutions of the indicial equations of (typically) much smaller degree dictated by the multiplicity of the head polynomial zeros of the linear ODE.

We now assume that the true singularities and their associated exponents have been determined. If $M>M_{0}$, then (A.6) is a constraint on the spurious singularity exponents and is of little interest. On the other hand if $M=M_{0}$, then there are no spurious singularities and the $x=x_{i}$ are either true singularities or apparent singularities. Furthermore, in many cases of interest as we found in our analysis of $\tilde{\chi}^{(3)}$ and $\tilde{\chi}^{(4)}$, the multiplicities of the apparent singularities at $x=x_{i}$ are all $q_{i}=1$ and the exponents are all $s_{i}=M_{0}$. Then the contribution to $\sum_{i}\left(s_{i}+q_{i}\left(q_{i}+1\right) / 2\right)$ coming from the apparent singularities is simply $\sum_{i}\left(s_{i}+1\right)=\sum_{i}\left(M_{0}+1\right)=D_{a p p} \cdot\left(M_{0}+1\right)$ with $D_{a p p}=D-D_{0}$ and $D_{0}$ the minimum possible degree of the ODE. The sum-rule (A.6) can now be rewritten as

$$
\begin{aligned}
& s_{0}+s_{\infty}+\sum_{i, \text { true }}\left(s_{i}+\frac{1}{2} q_{i} \cdot\left(q_{i}+1\right)\right)= \\
& M_{0} \cdot D-D_{\text {app }} \cdot\left(M_{0}+1\right)=M_{0} \cdot D_{0}-D_{\text {app }},
\end{aligned}
$$

which is an explicit formula for $D_{a p p}$ in terms of the true singularities only. To emphasize this point we note that for any physical problem the true singularities are determined and encoded in the generating function $S(x)$. By deciding to represent this information as a linear ODE of minimum order of the form (26) we are forced to specify a total of $\left(M_{0}+1\right)\left(D_{0}+D_{a p p}+1\right)$ coefficients with $D_{a p p}$ given by (A.7). For the $S=\tilde{\chi}^{(5)}$ example treated in the text, $s_{0}=192$ from Table 4 and $s_{\infty}=147$, which is 56 from Table 4 plus the 14 term sum $0+1+\cdots+13=91$ from the additional regular solution exponents inferred to be in the degree $M_{0}=33$ indicial equation. The remaining true singularity exponent sum in (A.7) is 653 from Table 4. On solving (A.7) for $D_{a p p}$ we get the value $D_{a p p}=1384$ given in the text. The number of ODE coefficients is $\left(M_{0}+1\right)\left(D_{0}+D_{a p p}+1\right)=(33+1)(72+1384+1)=49538$.

There are a number of related observations that are significant. Firstly, the presence of apparent singularities in the minimum order ODE implies that there are constraint conditions [30], namely $M_{0}-1$ conditions for each singularity in addition to the observed exponent value $s_{i}=M_{0}$. Thus $D_{a p p} \cdot M_{0}$ coefficients in total are fixed by the constraints and this means that in principle we need only $\left(M_{0}+1\right)\left(D_{0}+1\right)+D_{\text {app }}$ series terms to determine the minimum order ODE. In the $\tilde{\chi}^{(5)}$ example this is $(33+1)(72+1)+1384=3866$ which is much smaller than the $>7000$ terms for any $\tilde{\chi}^{(5)}$ ODE given in Table 1. Unfortunately, we know of no practical way to implement the constraints as they are in general non-linear. Specifically, let us write the minimum order ODE operator as $\sum_{m} f_{m}\left(x \cdot \frac{\mathrm{d}}{\mathrm{d} x}\right)^{M_{0}-m}$ with $f_{m}=0$ for $m>M_{0}$ 
and the head polynomial $f_{0}$ factored as $f_{0}=P_{t r u e} \cdot P_{a p p}$, thus clearly separating the true and apparent singularities. The apparent singularity constraint conditions are then the statement that each

$$
\begin{gathered}
F_{m}=\left[f_{m} \cdot\left(f_{m}+x \cdot \frac{\mathrm{d} f_{m-1}}{\mathrm{~d} x}\right)-f_{m-1} \cdot\left(f_{m+1}+x \cdot \frac{\mathrm{d} f_{m}}{\mathrm{~d} x}\right)\right] / P_{a p p}, \\
m=1,2, \cdots, M_{0},
\end{gathered}
$$

is a polynomial of degree $2 \cdot D_{0}+D_{a p p}$. It is the exact division and reduction in degree from $2\left(D_{0}+D_{a p p}\right)$ that implies the existence of $D_{a p p}$ conditions in the numerator of the right hand side of (A.8) for each individual $\mathrm{m}$.

Secondly, although the number of coefficients needed in an ODE can be dramatically reduced by moving away from minimum order $\sharp$, this is offset by a dramatic increase in the size of the integer coefficients specifying the ODE. This observation is based on our experience with a $6 \tilde{\chi}^{(3)}-\tilde{\chi}^{(1)}$ analysis. The minimum order ODE has $M_{0}=6$ and degree $D=40$. With the normalization choice $a_{M 0}=1$ in (26), the remaining coefficients are integers and can be found by a mod prime and Chinese remainder theorem analysis using five primes. In contrast, with the same normalization, the "best" choice ODE from Table 2 with $M=10, D=17$ has as its remaining coefficients rational fractions with numerator and denominator integers each typically 160 digits in length. To find these requires a supplementary continued fraction calculation starting from Chinese remainder theorem residues of 320 digits and requires about 70 primes. Similar results hold for other non-minimum order ODE's for $6 \tilde{\chi}^{(3)}-\tilde{\chi}^{(1)}$ and we believe analogous disparities are likely in any $2 \tilde{\chi}^{(5)}-\tilde{\chi}^{(3)}$ analysis. But we need to emphasize that the utility of the "best" mod prime ODE as a recursion relation device [24] remains. It enables us, for any given prime, to extend the shortest possible generating function series to the length necessary to find the minimum order ODE.

\section{Appendix B. On the Landau singularities}

The discussion of the Landau singularities of Ising like integrals in [14] is based on analogues of the $(2 n-2)$-dimensional integrals for $\tilde{\chi}^{(n)}$ given by Wu et al. [2]. Here we derive and extend those results based on analogs of the $(n-1)$-dimensional integrals (5). The calculations are complementary, each having certain advantages and disadvantages.

In the forthcoming technical discussion we will often use the following definitions supplementing those in (5-9). The square root factor appearing in $x_{i}$ and $y_{i}$ we denote by:

$$
f_{i}=\frac{1}{2 w} \cdot \sqrt{\left(1-2 w \cos \left(\phi_{i}\right)\right)^{2}-4 w^{2}}=1 / y_{i} .
$$

We also define

$$
\cos \left(\zeta_{i}\right)=1 /(2 w)-\cos \left(\phi_{i}\right), \quad \sin \left(\zeta_{i}\right)=\mathrm{i} \cdot f_{i}
$$

so that we can write:

$$
x_{i}=\exp \left(\mathrm{i} \zeta_{i}\right) .
$$

The formulae (B.1-B.3) are understood to apply for $s$ or $w$ small and elsewhere by analytic continuation. Furthermore we will take it to be understood that by the

$\sharp$ In the $\tilde{\chi}^{(5)}$ example we can move from the 49538 coefficients given above for $M=M_{0}=33$ to $(M+1)(D+1)=7410$ given in Table 1 for $M=56$. 
integral $\tilde{\chi}^{(n)}$ we mean (5) and various analytic continuations of the series analytic at $w=0$. This means we do not distinguish between "integral" and that "particular solution" of the linear ODE that agrees with (5) for small $w$ (or $s$ ) but whose domain is not restricted in any wayt. This is a great simplification for our discussion below since the value of $\tilde{\chi}^{(n)}\left(w_{c}\right)$ reached by analytic continuation in general depends on the path chosen for $w$ between 0 and $w_{c}$ and this can lead to very complicated topological considerations. We do not address any of that here but note that some of the differences in $\tilde{\chi}^{(n)}$ on different branches might arise because analytic continuation requires the displacement of branch cuts which result in changes in the signs of $f_{i}$ and $\zeta_{i}$ in (B.1-B.3). The results we describe below allow for all possible sign changes and thus the singularity list is the complete list covering all these local signs.

This must be borne in mind when, for example, we say $\tilde{\chi}^{(n)}$ is singular at certain points $|s|<1$ since it is certainly the case that the radius of convergence of $\tilde{\chi}^{(n)}$ is $|s|=1$. The latter observation follows trivially from the fact that if the phases in (5) are real then for $|s|<1$ one finds that $f_{i}$ cannot vanish and also $\left|x_{i}\right|<|s|$. When we want to restrict the domain on which the integral is defined to the region $|s| \leq 1$ (and on which for $|s|<1$ the series applies), we will explicitly indicate this by making reference to the "principal disc" or the "integral on the principal disc".

The points $s= \pm 1, \pm$ i or equivalently $w= \pm 1 / 4, \infty$ are fairly obvious singularities of $\tilde{\chi}^{(n)}$ and to simplify the analysis we explicitly exclude these points. A consequence of this exclusion is that (B.2) implies that if $\sin \left(\zeta_{i}\right)=\sin \left(\phi_{i}\right)=0$ then $w= \pm 1 / 4$ or $\infty$. Thus when we exclude these singular points we are preventing $\sin \left(\zeta_{i}\right)$ and $\sin \left(\phi_{i}\right)$ from vanishing simultaneously, a result we will use on a number of occasions below.

The discussion of the Landau singularities of an integral like (5) is somewhat simplified by the fact that the integration is over a unit cell of a periodic function and thus there are no end-point singularities. In fact it is best to think of the $\phi_{i}$ integrations as closed contour integrations in $z_{i}=\exp \left(\mathrm{i} \phi_{i}\right)$ that can be arbitrarily deformed away from the unit circle provided no integrand singularities are crossed while the constraint $\prod z_{i}=1$ is maintained. These singularities in (5) are at $f_{i}=0$ for all $i, x_{i} x_{j}=1$ for all $i \neq j$ and $\prod x_{i}=1$. They are not all independent since, for example, $f_{i}=0$ implies $\zeta_{i}=0$ or $\pi$ and $x_{i}= \pm 1$. Thus the vanishing of $f_{i}$ can overlap with the $x_{i}$ product singularities. However it is important to note that $f_{i}=0$, which is a condition on a single $\phi_{i}$, is very different from $\prod x_{i}=1$, which is a relationship between all $\phi_{i}$. If $f_{i}=0$ cannot be avoided by the $z_{i}$ contour for some particular $w$, then the integral $\tilde{\chi}^{(n)}$ is singular at that $w$. On the other hand $\prod x_{i}=1$ can lead to a singularity of the integral only if $\prod x_{i}$ is also stationary with respect to variation in all $\phi_{i}$ subject to the phase constraint in (9). Both $f_{i}=0$ and $\prod x_{i}=1$ are examples of pinch singularities, but to distinguish the simpler $f_{i}=0$ case we will refer to it exclusively as a pinch singularity in the discussion below. The $\prod x_{i}=1$ case or any similar situation in which a non-trivial stationary condition must also be satisfied we will call a van Hove singularity in recognition of his analysis [31] that predates that of Landau [32] by several years. Another singular integral situation arises when, say, $x_{1} x_{2}=1$ and $\prod x_{i}=1$ are simultaneously satisfied but rather than

$\dagger$ Thus completing the generalizations already in print. In [10] "integral" was understood to mean a single-valued function in the cut $w$ plane. The definition of "integral" was extended in [15] to be a single valued function in a cut $s$ plane and hence double-valued in $w$. Finally, in a Landau singularity analysis as in [14] one no longer attempts to specify on which local Riemann sheet any particular singularity occurs.

ब And is univalued! 
$x_{1} x_{2}$ and $\prod x_{i}$ being separately stationary the normals to these two hypersurfaces are parallel ([33], p.48). In this case the integration variables are trapped between two distinct hypersurfaces that touch tangentially.

Both to simplify the discussion and because this includes the most important situations, we start with the Landau problem of only $\prod x_{i}=1$ and $f_{i}=0$ as singularities in the integrand of $\tilde{\chi}^{(n)}$. Since $x_{i}=\exp \left(\mathrm{i} \zeta_{i}\right)$ this product constraint together with the phase constraint in (9) results in the symmetric pair

$$
\sum \phi_{i}=0 \quad \bmod 2 \pi, \quad \sum \zeta_{i}=0 \quad \bmod 2 \pi .
$$

The phase constraint we handle directly by taking $\phi_{i}, i=1, \cdots, n-1$, as independent so that $\zeta_{i}=\zeta_{i}\left(\phi_{i}\right), i=1, \cdots, n-1$, and $\zeta_{n}=\zeta_{n}\left(\phi_{n}\right)=\zeta_{n}\left(2 \pi k-\phi_{1}-\cdots-\phi_{n-1}\right)$. The requirement that $\sum \zeta_{i}$ be stationary with respect to phase variation is that the derivative combinations $\zeta_{i}^{\prime}-\zeta_{n}^{\prime}$ vanish where $i=1, \cdots, n-1$ and $\zeta_{i}^{\prime}=\partial \zeta_{i} / \partial \phi_{i}=$ $-\sin \left(\phi_{i}\right) / \sin \left(\zeta_{i}\right)$. The last equality follows from the definition (B.2). The derivative conditions can be rewritten as

$$
\sin \left(\zeta_{i}\right) \cdot \sin \left(\phi_{j}\right)=\sin \left(\zeta_{j}\right) \cdot \sin \left(\phi_{i}\right), \quad i \neq j
$$

and our derivation requires that (B.5) be subject to the restriction that no $\sin \left(\zeta_{i}\right)$ vanishes. However this restriction can be dropped because we are looking only for solutions for which $\sin \left(\phi_{i}\right)$ and $\sin \left(\zeta_{i}\right)$ are not simultaneously zero and the case that (B.5) yields all $\sin \left(\zeta_{i}\right)=0$ is nothing but the pinch singularity condition we must investigate also. We can rewrite (B.5) for each $i, j$ combination as the pair

$$
\begin{aligned}
& \left(\cos \left(\phi_{i}\right)-\cos \left(\phi_{j}\right)\right) \cdot\left(4 w-\cos \left(\phi_{i}\right)-\cos \left(\phi_{j}\right)+4 w \cdot \cos \left(\phi_{i}\right) \cos \left(\phi_{j}\right)\right)=0, \\
& \left(\cos \left(\zeta_{i}\right)-\cos \left(\zeta_{j}\right)\right) \cdot\left(4 w-\cos \left(\zeta_{i}\right)-\cos \left(\zeta_{j}\right)+4 w \cdot \cos \left(\zeta_{i}\right) \cos \left(\zeta_{j}\right)\right)=0
\end{aligned}
$$

by squaring, rewriting the sine functions in terms of cosines, and utilizing the definition (B.2) which we reproduce here in symmetric form

$$
\cos \left(\phi_{i}\right)+\cos \left(\zeta_{i}\right)=1 /(2 w), \quad i=1 \ldots n .
$$

Note that (B.6) and (B.7) allow all possible sign combinations $\pm \phi_{j}, \pm \zeta_{j}$ for a given $\phi_{i}, \zeta_{i}$ pair and one must in all cases check that (B.5) is also satisfied. That there is a remaining sign degeneracy allowed by (B.5) is a consequence of our decision to define our integral to include all possible sign combinations in the local Riemann sheet. If the domain of $w$ were to be restricted to a particular Riemann sheet then additional analysis would be required to determine the uniquely signed solution.

The equations (B.4-B.7) are the Landau conditions for our reduced problem of $\prod x_{i}=1$ and $f_{i}=0$ as the only singularities. We now consider specific situations, Case 1 to Case 5 .

\section{Appendix B.1. Case 1: the phases $\phi_{i}$ and $\zeta_{i}$ equal 0 or $\pi$}

The simplest case is that of phases $\phi_{i}$ and $\zeta_{i}$ equal 0 or $\pi$. The stationary constraint (B.5) is trivially satisfied and (B.7) implies singularities at $w= \pm 1 / 4$ or $\infty$. These are the points we excluded from the analysis and we again note that $\sin \left(\phi_{i}\right)$ and $\sin \left(\zeta_{i}\right)$ cannot simultaneously vanish.

Appendix B.2. Case 2: all $\phi_{i}$ equal and all $\zeta_{i}$ equal

Equally simple is the case of all $\phi_{i}$ equal and all $\zeta_{i}$ equal. Again the stationary constraint (B.5) or (B.6) is satisfied automatically and the phase constraints (B.4) are 
satisfied with

$$
\phi_{i}=2 \pi k / n, \quad \zeta_{i}=2 \pi m / n, \quad 0 \leq k, m<n
$$

and with $\phi_{i}$ and $\zeta_{i}$ not both 0 or $\pi$ so as to exclude Case 1 . The associated singularities are at

$$
1 /\left(2 w_{k m}\right)=s_{k m}+1 / s_{k m}=\cos (2 \pi k / n)+\cos (2 \pi m / n) .
$$

Because the stationary constraint for these singularities is automatic the full Landau formalism is not necessary and (B.8), (B.9) could have been guessed just as they were in [3]. Note that $\left|s_{k m}\right|=1$ and the calculation in [3] shows them to be principal disc singularities. In the following we will designate them as Nickelian singularities to distinguish them from other van Hove singularities. The singularities $w_{k m}$ can be given as the roots of polynomials in $w$ with integer coefficients.

Remark 1 A new situation not considered in [3] arises out of the Landau formalism. For a given $\phi_{i}, \zeta_{i}$ combination, the constraints (B.5-B.7) allow $\phi_{j}, \zeta_{j}$ to be $-\phi_{i},-\zeta_{i}$ in addition to the $\phi_{i}, \zeta_{i}$ we have considered. For every such sign reversal there is one pairwise cancellation in the constraint sums (B.4) so that we should add to (B.8), (B.9) new singularity conditions obtained by the replacements $n \rightarrow n-2$, $n \rightarrow n-4$, etc. We will however leave (B.8), (B.9) unchanged and if there is a possibility of confusion, refer explicitly to (B.8), (B.9), for which there has been no pairwise cancellation, as the "irreducible" conditions. The $n \rightarrow n-2 m$ replacement singularities will be treated separately as Case 5 below as this situation arises numerous times.

Remark 2 A very important observation concerning the $n \rightarrow n-2 m$ replacement singularities is that none of them are principal disc singularities. To see this note that (B.1), (B.2) defines $\zeta_{j}$ as an even function of $\phi_{j}$ for $|s|<1$ on the principal disc and by continuity to the limiting case $|s|=1$ as well. On the other hand, for fixed $\phi_{i}, \zeta_{i}$, (B.5) requires $\zeta_{j}$ to be an odd function of $\phi_{j}$. Thus while (B.5) allows $\phi_{j}, \zeta_{j}$ to be $-\phi_{i}$, $-\zeta_{i}$ in addition to the $\phi_{i}, \zeta_{i}$, this singularity cannot be on the principal disc but rather must be on those other "Riemann sheets" on which the $f_{j}$ square root function has the opposite sign. The numerical evidence is consistent with this result. It was already shown in [3] that $\tilde{\chi}^{(3)}$ principal disc singularities were not present on the principal $\operatorname{disc}$ of $\tilde{\chi}^{(5)}$.

Appendix B.3. Case 3: all $\sin \left(\zeta_{i}\right)=0$, i.e. all $f_{i}=0$, so that $\zeta_{i}=0$ or $\pi$

At the next level of complexity consider the possibility that all $\sin \left(\zeta_{i}\right)=0$, i.e. all $f_{i}=0$, so that $\zeta_{i}=0$ or $\pi$. The constraint (B.5), which in this case specifies a pinch singularity, is trivially satisfied. We find from (B.7) that there are only two possible values for $\cos \left(\phi_{i}\right)$, namely $\cos \left(\phi^{(+)}\right)=1 /(2 w)+1$ and $\cos \left(\phi^{(-)}\right)=1 /(2 w)-1$. The former is associated with $\zeta_{i}=\pi$ and if we demand that the singularity condition in (B.4) also be satisfied then we must have an even number of these terms. We implement the phase constraint (B.4) on $\phi_{i}$ as follows. Since there are only two possible valuest we can write $k \phi^{(+)}+(n-k) \phi^{(-)}=0 \bmod 2 \pi$ and from the preceding remarks, $k$ is even. Equivalently, $\exp \left(\mathrm{i} k \phi^{(+)}+\mathrm{i}(\mathrm{n}-\mathrm{k}) \phi^{(-)}\right)=1$ or $\exp \left(\mathrm{i} k \phi^{(+)}\right)=\exp \left(-\mathrm{i}(n-k) \phi^{(-)}\right)$. Now add to this last equation the reciprocal and obtain $\cos \left(k \phi^{(+)}\right)=\cos \left((n-k) \phi^{(-)}\right)$. This form is convenient because each $\cos (m \phi)$

$\ddagger$ Exactly as in Case 2 we consider only the "irreducible" case in which a given value of $\cos \left(\phi_{i}\right)$ defines a uniquely signed $\phi_{i}$ and hence there are no pairwise cancellations in the constraint $\sum \phi_{i}$. We will remark further on the general situation, considered as Case 5, later. 
can be expressed simply in terms of $\cos (\phi)$. On using the definition of Chebyshev polynomials we get

$$
\begin{array}{r}
T_{k}\left(1 /\left(2 w_{k}\right)+1\right)=T_{n-k}\left(\left(1 /\left(2 w_{k}\right)-1\right),\right. \\
0<k<n, \quad \text { and } \quad k \text { even, }
\end{array}
$$

as the defining equation(s) for the singularities. Note that we have excluded $k=0$ or $n$ since all $\phi_{i}$ are equal and (B.10) becomes a special case of (B.8), (B.9).

Remark 3 The singularity conditions (B.10) do not allow for singularities on $|s|=1$, as we now show. The condition $|s|=1$ is the condition $w$ real and $-2<1 /(2 w)<2$. Consider first the interval $0<1 /(2 w)<2$. Then the argument of $T_{k}$ in (B.10) lies between 1 and 3 and $\left|T_{k}\right|>1$, while the argument of $T_{n-k}$ lies between -1 and 1 and $\left|T_{n-k}\right| \leq 1$. Thus (B.10) cannot be satisfied. A similar argument applies on the interval $-2<1 /(2 w)<0$. Since we excluded $w= \pm 1 / 4$ and $\infty$ at the outset, the proof is complete.

Remark 4 The same integral singularity conditions (B.10), but with odd index $k$ allowed as well, were given in [16] based on the integrand singularity condition $f_{i}=0$ for all $i$ irrespective of $\prod x_{i}$. We see here the partial overlap between the all $f_{i}=0$ and $\prod x_{i}=1$ conditions, but because $f_{i}=0$ is the more general condition it would appear that odd $k$ should be included in (B.10). In fact (B.10) is correct because of the very special structure of the Ising integrals (5). This will be proved below. But first note that the integrals evaluated in [13], where the integrand in (5) was replaced by the simpler pole product $\left(\prod y_{i}\right)^{2}$, all show that both odd and even $k$ are required in that case. That is to say, for these integrals the pinch singularity conditions are both necessary and sufficient and the same should apply also to the Ising $\tilde{\chi}^{(n)}$. The only way contours that are trapped in the process of analytic continuation in the toy integrals could escape being trapped in $\tilde{\chi}^{(n)}$ is for at least one $f_{i}$ factor to become nonsingular. The mechanism for this to happen is that in the vicinity of a potential pinch an $f_{i}$ appears not in a Laurent series but rather in a Taylor series in powers of $\left(f_{i}\right)^{2}$. Given the complexity of the Ising $\tilde{\chi}^{(n)}$ integrand this would seem rather miraculous but in fact happens as we now show. Not too surprisingly, this point was entirely missed in [16].

The argument below is for a pinch singularity defined by $f_{i}=0$ for all $i$. We begin by noting that at a point where $f_{i}$ is small, $\zeta_{i}$ is either small or near $\pi$. For purposes of the present argument only, we replace those $\zeta_{i}$ near $\pi$ by $\zeta_{i}-\pi$ and incorporate this change consistently by the simultaneous replacement $x_{i} \rightarrow-x_{i}$. The factor $\left(1+\prod x_{i}\right) /\left(1-\prod x_{i}\right)$ in the $\tilde{\chi}^{(n)}$ integrand can then be written as $\cot \left(\sum \zeta_{i} / 2\right)$ if an even number of such replacements were made or $\tan \left(\sum \zeta_{i} / 2\right)$ if an odd number of such replacements were made. In the first case, only a Laurent expansion is possible and the pinch singularity is qualitatively like that in the toy integral with integrand $\left(\prod y_{i}\right)^{2}$. Since that integral was observed to have the singularities (B.10) the same must be true here and we conclude that the necessary conditions (B.10) are also sufficient for an integral singularity.

However, in the odd replacement case a Taylor expansion in odd powers of $\sum \zeta_{i}$ exists. This will yield a sum of terms of the form $\prod\left(\zeta_{i}\right)^{p_{i}}$ with with $\sum p_{i}$ odd. Since $f_{i}=\mathrm{i} \cdot \sin \left(\zeta_{i}\right)$ implies $\zeta_{i}$ is an odd function of $f_{i}$ the expansion could equally well be written as a sum of $\prod\left(f_{i}\right)^{p_{i}}$ with again $\sum p_{i}$ odd. Because $\sum p_{i}$ is odd there is at least one $p_{i}$ which is odd and of course this $p_{i}>0$. Now recall that the $\tilde{\chi}^{(n)}$ integrand also contains the factor $\prod y_{i}=\prod\left(1 / f_{i}\right)$ and this shifts every $p_{i}$ down by one. In particular the original odd $p_{i}$ has now become even and is still non-negative so that 
the integration over the analytically continued $\phi_{i}$ encounters no singularity, i.e. no pinch. This completes the argument for those proxy Ising integrals that do not contain the $\left(G^{(n)}\right)^{2}$ factor.

To show that the presence of $\left(G^{(n)}\right)^{2}$ in the integrand does not change the argument for an absence of singularities we first note that $G^{(n)}$ is a product of terms each of which is of the form

$$
2 \sin \left(\left(\phi_{i}-\phi_{j}\right) / 2\right) \cdot \frac{\sqrt{x_{i} x_{j}}}{\left(1-x_{i} x_{j}\right)} .
$$

The $x_{i} x_{j}$ dependent factor is proportional to either $\sin \left(\left(\zeta_{i}+\zeta_{j}\right) / 2\right)$ or $1 / \sin \left(\left(\zeta_{i}+\zeta_{j}\right) / 2\right)$, after making the replacements described in the penultimate paragraph, so that now $\zeta_{i}$ and $\zeta_{j}$ are understood to always be near zero. The sine function in the numerator of (B.11) is crucial in canceling a potential zero in the denominator, but has a complicated representation in terms of $\zeta_{i}$ and $\zeta_{j}$. For purposes of a singularity existence analysis it is legitimate to replace it by any more convenient analytic and asymptotically linear $\S$ function in the neighbourhood of $\zeta_{i}=\zeta_{j}=0$ and we choose instead of $\sin \left(\left(\phi_{i}-\phi_{j}\right) / 2\right)$ the difference $\left(f_{j}\right)^{2}-\left(f_{i}\right)^{2}=\sin ^{2}\left(\zeta_{i}\right)-\sin ^{2}\left(\zeta_{j}\right)$. Then (B.11) is proportional to one of

$$
\begin{aligned}
& \left(\sin ^{2} \zeta_{i}-\sin ^{2} \zeta_{j}\right) \sin \left(\left(\zeta_{i}+\zeta_{j}\right) / 2\right) \\
& \left(\sin ^{2} \zeta_{i}-\sin ^{2} \zeta_{j}\right) / \sin \left(\left(\zeta_{i}+\zeta_{j}\right) / 2\right)= \\
& 2\left(\sin \zeta_{i}-\sin \zeta_{j}\right) \cdot \cos \left(\left(\zeta_{i}-\zeta_{j}\right) / 2\right) .
\end{aligned}
$$

Both of the expressions in (B.12) have a Taylor expansion with terms of the form $\left(\zeta_{i}\right)^{p_{i}}\left(\zeta_{j}\right)^{p_{j}}$ with $p_{i}+p_{j}$ odd. An equivalent expansion is in terms $\left(f_{i}\right)^{p_{i}}\left(f_{j}\right)^{p_{j}}$ with $p_{i}+p_{j}$ again odd. The square of this expansion will be similar but with $p_{i}+p_{j}$ now even. Finally the expansion of $\left(G^{(n)}\right)^{2}$ must be a sum of terms of the form $\prod\left(f_{i}\right)^{p_{i}}$ with $\sum p_{i}$ even and multiplying the expansion obtained in the absence $\left(G^{(n)}\right)^{2}$ by terms $\prod\left(f_{i}\right)^{p_{i}}$ for which $\sum p_{i}$ is even cannot change the argument for the absence of a pinch.

To summarize this case, the pinch conditions give (B.10) as necessary. The evidence from the toy model study [13] is almost certainly a proof that (B.10) is also sufficient for these singularities of $\tilde{\chi}^{(n)}$.

Remark 5 A situation closely related to $\sin \left(\zeta_{i}\right)=0$, for all $i$, as treated above is $\sin \left(\phi_{i}\right)=0$, for all $i$. This leads to van Hove singularities in which the stationary conditions (B.5) and both conditions (B.4) must be satisfied. Each $\phi_{i}$ is either 0 or $\pi$. The phase constraint then requires an even number of $\phi_{i}=\pi$ values. On imposing the singularity constraint in (B.4) the same solution (B.10) is obtained.

Remark 6 This equality of solutions for all $\sin \left(\zeta_{i}\right)=0$ and all $\sin \left(\phi_{i}\right)=0$ is expected and illustrates a very important aspect of the Landau analysis of the integral representation (5) for $\tilde{\chi}^{(n)}$. The original representation for $\tilde{\chi}^{(n)}$ given in [2] is an integral over two sets of $n-1$ independent phases with an integrand that is symmetric under the interchange of these sets $\|$. Integrating out one set to arrive at (5) has obviously broken this symmetry, but a vestige of it remains in the two conditions (B.4). The $\phi_{i}$ are the remaining dummy phase variables of integration while the $\zeta_{i}=\zeta_{i}\left(\phi_{i}\right)$ are the specific values that result from evaluating the other set

$\S$ Linear in the deviation of $\phi_{i}$ from the singular point. This means a linear function in the quadratic $f_{i}^{2}$.

$\|$ The invariance under phase variable interchange is not always obvious in a particular formula. See the remarks in $\mathrm{Wu}$ et al. [2] following their equation (4.87) on this point. 
of phases at the $\mathrm{Wu}$ et al. [2] integral singular points. Finding singularities of the integral such as (B.10) amounts to completing the integration process and while the steps associated with dealing with the "integrated" $\zeta_{i}$ versus the "unintegrated" $\phi_{i}$ are obviously different, once the $\phi_{i}$ values have been fixed by the Landau conditions the symmetry is restored and the final answers one obtains must be the same. That is, for every combination of the $\zeta_{i}, \phi_{j}$ leading to an integral singularity there is another set $\phi_{i}, \zeta_{j}$ obtained by $\zeta \leftrightarrow \phi$ interchange that leads to the same singularity and we can always choose the combination that requires the least computational effort.

Remark $\mathbf{7}$ The above comparison of alternatives also illustrates that there is no fundamental distinction between the pinch and van Hove singularities. Rather it is just a distinction in the ordering of the steps of the calculation. This is particularly relevant when comparing the calculations here to those in [13]. There the Landau analysis is done at the $(2 n-2)$ integral stage and there is no analogue of the pinch singularity in the restricted sense we have defined here. Instead, every singularity evaluation requires a supplementary stationary condition evaluation and thus all singularities are of van Hove type.

This concludes our treatment of the singularities associated with the pinch condition $\sin \left(\zeta_{i}\right)=0$ and the corresponding special van Hove case $\sin \left(\phi_{i}\right)=0$. This list is exhaustive as there can be no integral singularities for the mixed case that $\sin \left(\zeta_{k}\right)=0, k=1, \cdots, m$, and $\sin \left(\zeta_{j}\right) \neq 0, j=m+1, \cdots, n$, with $1 \leq m<n$. To see this note that while the $\zeta_{k}$ is a singular, i.e. square root, function of $\phi_{k}$ in the neighbourhood of $\sin \left(\zeta_{k}\right)=0$, the $\phi_{k}$ is an analytic function of $\zeta_{k}$. Specifically, $\phi_{k}$ is asymptotically a constant with an added quadratic dependence on the deviation $\delta \zeta_{k}$ of $\zeta_{k}$ from 0 or $\pi$. If, for each $k, 1 \leq k \leq m$, we replace $\int d \phi_{k} / \sin \left(\zeta_{k}\right) \simeq \int d \phi_{k} / f_{k}$ by $-\int d \zeta_{k} / \sin \left(\phi_{k}\right)$ then the only integrand singularity condition remaining is $\sum \zeta_{i}=0$ $\bmod 2 \pi$. Furthermore, because we are explicitly demanding $\sin \left(\zeta_{n}\right) \neq 0, \zeta_{n}\left(\phi_{n}\right)$ is an analytic function in the neighbourhood of the integrand singularity. As before we eliminate $\phi_{n}$ using the constraint $\sum \phi_{i}=0 \bmod 2 \pi$ except that here we also express $\phi_{k}$ as $\phi_{k}\left(\zeta_{k}\right)$ for $1 \leq k \leq m$. The leading dependence of $\zeta_{n}$ on $\zeta_{k}$ is the quadratic dependence $\delta \zeta_{k}^{2}$ and this implies that no variation such as $\partial \sum \zeta_{i} / \partial \zeta_{k}=$ $\partial \zeta_{k} / \partial \zeta_{k}-\partial \zeta_{n} / \partial \zeta_{k}=1-$ constant $\delta \zeta_{k} \zeta_{n}^{\prime}$ can ever vanish $\dagger$. That is, no integral singularity is possible in the mixed case.

\section{Appendix B.4. Case 4: neither $\sin \left(\phi_{i}\right)$ nor $\sin \left(\zeta_{i}\right)$ vanish}

In all of the above cases the Landau stationary condition was either automatic because of symmetry or did not need to be invoked because the same singularities arose from the $f_{i}=0$ for all $i$ condition. References $[13,14]$ showed how other singularities can arise out of the Landau rules for some toy integrals and we give in this section the first and simplest example of a case where the Landau stationary conditions are non-trivial for the Ising $\tilde{\chi}^{(n)}$. Consider the situation $\ddagger$ that in (B.4-B.7) $k$ of the $\phi_{i}, \zeta_{i}$ take on the values $\phi_{a}, \zeta_{a}$ and the remaining $(n-k)$ are $\phi_{b}, \zeta_{b}$. The phase constraints can be written as polynomial relations in cosines as in (B.10) but with the difference that the individual terms like $\cos \left(\phi_{a}\right)$ are not explicitly given as functions of $w$ but are to be determined self-consistently together with $w$. In summary we must solve the analogue

$\dagger$ That $\zeta_{n}^{\prime}$ is finite is crucial. This fails in the previous pinch situation where all $\sin \left(\zeta_{i}\right)=0$.

$\ddagger$ As in Cases 2 and 3 we restrict our attention to the "irreducible" situation in which there is no cancellation between pairs of phases differing only in sign. The general situation is treated as Case 5 . 
of (B.10) which is the pair

$$
\begin{aligned}
& T_{k}\left(\cos \phi_{a}\right)=T_{n-k}\left(\cos \phi_{b}\right), \\
& T_{k}\left(\cos \zeta_{a}\right)=T_{n-k}\left(\cos \zeta_{b}\right), \quad 0<k<n
\end{aligned}
$$

together with the stationary constraints (B.6)

$$
\begin{aligned}
& 4 w-\cos \phi_{a}-\cos \phi_{b}+4 w \cdot \cos \phi_{b} \cos \phi_{a}=0, \\
& 4 w-\cos \zeta_{a}-\cos \zeta_{b}+4 w \cdot \cos \zeta_{b} \cos \zeta_{a}=0,
\end{aligned}
$$

and the definitions (B.7)

$$
\cos \phi_{a}+\cos \zeta_{a}=1 /(2 w), \quad \cos \phi_{b}+\cos \zeta_{b}=1 /(2 w)
$$

We can reduce these equations to somewhat simpler form by using (B.14) to eliminate $\phi_{b}$ and $\zeta_{b}$. The result is a triplet of equations

$$
\begin{aligned}
& T_{k}\left(\cos \phi_{a}\right)=T_{n-k}\left(\left(4 w-\cos \phi_{a}\right) /\left(1-4 w \cos \phi_{a}\right)\right), \\
& T_{k}\left(\cos \zeta_{a}\right)=T_{n-k}\left(\left(4 w-\cos \zeta_{a}\right) /\left(1-4 w \cos \zeta_{a}\right)\right), \\
& \cos \phi_{a}+\cos \zeta_{a}=1 /(2 w)
\end{aligned}
$$

defining the van Hove singularities when neither $\sin \left(\phi_{i}\right)$ nor $\sin \left(\zeta_{i}\right)$ vanish.

To produce the singularity polynomials, we use the last condition in (B.16) to eliminate $\cos \left(\zeta_{a}\right)$. Then, the elimination of $\cos \left(\phi_{a}\right)$ from the first two polynomial conditions in (B.16) gives the roots that are inserted into (B.16) to determine which are actually solutions. We must also go back and verify that (B.5) is satisfied as our candidate polynomials are all based on the squared form of (B.5) as expressed in (B.6), (B.7).

Our explicit procedure for eliminating the phase variables in (B.16) is as follows. The first condition in (B.16) can be expressed as a polynomial equation $p_{1}\left(\cos \left(\phi_{a}\right)\right)=$ 0 . The second condition can be reduced to another $p_{2}\left(\cos \left(\phi_{a}\right)\right)=0$ if we use the last condition to eliminate $\cos \left(\zeta_{a}\right)$. We are not interested in the solutions $\cos \left(\phi_{a}\right)= \pm 1$ as these are a subset of those already found. We therefore take it as given that $p_{1}$ and $p_{2}$ are reduced polynomials which do not contain the factors $\cos \left(\phi_{a}\right)= \pm 1$. They are both of degree $m=n-2, n-1, n$, depending on whether $k$ and $n-k$ are even-even, odd-even or odd-odd combinations. We now eliminate $\cos \left(\phi_{a}\right)$ by the following iterative process. We first reduce the algebraic complexity of the polynomials $p_{1}$ and $p_{2}$ by dividing them at each stage by their greatest common divisor. Assume this has been done and they are now written as $p_{1}=a_{1}+\cdots+b_{1} \cos ^{m}\left(\phi_{a}\right), p_{2}=a_{2}+\cdots+b_{2} \cos ^{m}\left(\phi_{a}\right)$. We then generate two new polynomials $q_{1}=\left(A_{2} p_{1}-A_{1} p_{2}\right) / \cos \left(\phi_{a}\right)$ and $q_{2}=B_{2} p_{1}-B_{1} p_{2}$ where $A_{1}$ and $A_{2}$ are just $a_{1}$ and $a_{2}$ divided by their greatest common divisor and similarly for $B_{1}$ and $B_{2}$. By construction $q_{1}$ and $q_{2}$ are of degree one less than $p_{1}$ and $p_{2}$ and after division by their greatest common divisor are the $p_{1}$ and $p_{2}$ of the next iteration. The process stops when we reach degree 0 . The greatest common divisors are polynomials in $w$ whose zeros are candidates for solutions of the original equations.

Remark 8 We find empirically that for given $n$ and $k$ in (B.16) the degree of the $w$ polynomials giving the Landau singularities is bounded by $n(n-2) / 4-m(m-1) / 2$, $m=\min (k, n-k)$. There is evidence from [14] based on toy integrals that the predicted singularities are seen in the ODE so that just as in Case 3 we conjecture that our polynomial solutions for Case 4 are both necessary and sufficient. For $n=2 N+1$, the number of $s$ plane singularities is $N(N+3), N(3 N+1)$ and $5 N\left(N^{2}-1\right) / 3$ for Cases 2-4 respectively. The corresponding number of $s$ plane singularities for $n=2 N$ 
is $2[(N-1)(N+3) / 2], 6\left[(N-1)^{2} / 2\right]$ and $2[(N-2)(2 N-3)(5 N+4) / 12]$ barring accidental degeneracies§.

Remark 9 As continuation of our empirical observations we note that of the Case 4 singularities, $N(N-1)$ are on $|s|=1$ for $n=2 N+1$ and $2\left[(N-2)^{2} / 2\right]$ for $n=2 N$. However, in none of these cases is the singularity on the principal disc of $\tilde{\chi}^{(n)}$ and the general proof that no Case $4,|s|=1$, singularity lies on the principal disc is as follows. Observe first that a singularity with $|s|=1$ on the principal disc requires that the integrand of $\tilde{\chi}^{(n)}$ be singular for real phases $\phi_{i}$. That is, both $\phi_{a}$ and $\phi_{b}$ must be real. Equivalently $\cos \left(\phi_{a}\right)$ and $\cos \left(\phi_{b}\right)$ are real and of magnitude less than one. Furthermore, the cosines are related by (B.14) or $\cos \left(\phi_{b}\right)=\left(4 w-\cos \left(\phi_{a}\right)\right) /\left(1-4 w \cos \left(\phi_{a}\right)\right)$. If $4 w$ is real and $|4 w|>1$, which is the condition for $|s|=1$, then $\left|\cos \left(\phi_{a}\right)\right|<1$ yields $\left|\cos \left(\phi_{b}\right)\right|>1$ and vice versa. An important consequence of this proof is that there are no Case 4 singularities to cancel any of the Case 2 Nickelian singularities.

Although we started the discussion of Case 4 with the remark that our calculation was just the first and simplest example of a non-trivial van Hove singularity, one observes that the singularity conditions (B.6), for example, require $\cos \left(\phi_{j}\right)=\cos \left(\phi_{i}\right)$ or $\cos \left(\phi_{j}\right)=\left(4 w-\cos \left(\phi_{i}\right)\right) /\left(1-4 w \cos \left(\phi_{i}\right)\right)$ and the latter is an example of a one to one Möbius' mapping. Thus it is not possible, for any given $w$, to have more than two distinct values in the set $\cos \left(\phi_{i}\right), i=1, \cdots, n$. This in turn implies that as long as we consider $\prod x_{i}=1$ as the only singularity we have exhausted all possibilities for the values of $\cos \left(\phi_{i}\right)$ in Cases 2-4 above. Of course this applies only to the "irreducible" situations and we turn now to the implications of the $\pm \phi_{i}$ and $\pm \zeta_{i}$ sign degeneracy.

\section{Appendix B.5. Case 5: $\pm \phi_{i}$ and $\pm \zeta_{i}$ sign degeneracy}

Once the "irreducible" solutions Cases 2-4 are known, all other solutions can be obtained by recursion which we now describe. Suppose the combinations $S^{(n)}=$ $\left\{\left(\phi_{i}^{(n)}, \zeta_{i}^{(n)}\right), i=1, \cdots, n\right\}$ are the stationary singular points of the integrand of $\tilde{\chi}^{(n)}$. Then we can construct a stationary singular point of the $\tilde{\chi}^{(n+2)}$ integrand as $S^{(n+2)}=\left\{S^{(n)},\left(\phi_{j}^{(n)}, \zeta_{j}^{(n)}\right),\left(-\phi_{j}^{(n)},-\zeta_{j}^{(n)}\right)\right\}$, where $j$ is any one of the $n$ values in $S^{(n)}$. Clearly by adding a pair with opposite signs we have guaranteed that the phase constraints (B.4) are satisfied. Furthermore, all constraint equations involving only cosines remain unchanged and thus satisfied. Finally, the stationary condition $\sin \left(\zeta_{i}\right) \sin \left(\phi_{j}\right)=\sin \left(\phi_{i}\right) \sin \left(\zeta_{j}\right)$ is potentially satisfied under the simultaneous sign change of $\phi_{j}$ and $\zeta_{j}$. Whether this occurs will depend on which Riemann sheet one is on. As an important example, and previously discussed in Case 2, on the principal disc for $|s| \leq 1$ the definitions (B.1, B.2) show that $\sin \left(\zeta_{j}\right)$ is an even function of $\phi_{j}$ and the new stationary condition generated by recursion cannot be satisfied. Specifically, the recursion mechanism explicitly excludes the possibility of addition to, or cancellation of, Case 2 "irreducible" singularities on the principal disc and leaves the argument that $|s|=1$ is a natural boundary of $\tilde{\chi}$ secure.

In conclusion, the Landau conditions for a singularity of $\tilde{\chi}^{(n)}$ are also the conditions $\|$ for that singularity in $\chi^{(n+2 k)}, k \geq 1$. Thus for a complete picture of the

$\S N=6$ affords examples. Here the $[k, m]$ combinations $[0,3]$ and $[2,2]$ in $\cos (k \pi / N)+\cos (m \pi / N)$ yield the same $1 /(2 w)=1$. Other degenerate combinations are $[0,4]$ and $[2,3]$ and those obtained by the replacements $k \rightarrow 6-k, m \rightarrow 6-m$.

$\|$ As always, these conditions are necessary but not sufficient. The numerical evidence from the 
singularities of $\tilde{\chi}^{(n)}$ it is sufficient to list the polynomials defining the "irreducible" singularities for the Cases 2-4.

\section{Appendix C. Singularities}

The following is a listing of the polynomials defining the Landau singularities of $\tilde{\chi}^{(n)}$ for small $n$ for the three Cases 2-4 discussed in Appendix B. Our notation for the polynomials from $\tilde{\chi}^{(n)}$ is $P\left({ }^{\text {Case }} n\right)$ or $P\left({ }^{\text {Case }} n_{n-k, k}\right)$ with $k$ defined in eqns. (B.10) and (B.16). We do not include the physical singularities $w= \pm 1 / 4$ or the simplest unphysical singularities at $w=0$ or $\infty$. We also do not give the (reducible) Case 5 , which would just be the list of $m>0, \tilde{\chi}^{(n-2 m)}$ polynomials for any given $\tilde{\chi}^{(n)}$. An example of the notation we use in the text in this situation is $P\left({ }^{5} 7 /{ }^{3} 3_{1,2}\right)$ to indicate a Case $3, n-2 m=3$ contribution to $\tilde{\chi}^{(7)}$.

Case 2. Circle singularities $P\left({ }^{2} n_{n, 0}\right)=P\left({ }^{2} n\right)$.

$$
\begin{aligned}
P\left({ }^{2} 3\right)= & (1+2 w)(1-w), \\
P\left({ }^{2} 4\right)= & \left(1-4 w^{2}\right), \\
P\left({ }^{2} 5\right)= & (1+w)\left(1+2 w-4 w^{2}\right)\left(1-3 w+w^{2}\right), \\
P\left({ }^{2} 6\right)= & \left(1-4 w^{2}\right)\left(1-9 w^{2}\right)\left(1-w^{2}\right), \\
P\left({ }^{2} 7\right)= & \left(1+2 w-8 w^{2}-8 w^{3}\right)\left(1+2 w-w^{2}-w^{3}\right)\left(1-5 w+6 w^{2}-w^{3}\right), \\
P\left({ }^{2} 8\right)= & \left(1-8 w^{2}\right)\left(1-2 w^{2}\right)\left(1-4 w^{2}\right)\left(1-12 w^{2}+4 w^{4}\right), \\
P\left({ }^{2} 9\right)= & (1+2 w)(1-w)\left(1-12 w^{2}+8 w^{3}\right)\left(1-6 w+9 w^{2}-w^{3}\right) \\
& \times\left(1-3 w^{2}-w^{3}\right)\left(1+3 w-w^{3}\right), \\
P\left({ }^{2} 10\right)= & \left(1-w^{2}\right)\left(1-5 w^{2}\right)\left(1-12 w^{2}+16 w^{4}\right) \\
& \times\left(1-7 w^{2}+w^{4}\right)\left(1-15 w^{2}+25 w^{4}\right) .
\end{aligned}
$$

Case 3. $f_{i}=0$ or $\cos \phi_{+}=1 / 2 w+1, \cos \phi_{-}=1 / 2 w-1$.

$$
\begin{aligned}
& P\left({ }^{3} 3_{1,2}\right)=\left(1+3 w+4 w^{2}\right), \\
& P\left({ }^{3} 4_{2,2}\right)=1, \\
& P\left({ }^{3} 5_{3,2}\right)=\left(1-7 w+5 w^{2}-4 w^{3}\right), \\
& P\left({ }^{3} 5_{1,4}\right)=\left(1+8 w+20 w^{2}+15 w^{3}+4 w^{4}\right), \\
& P\left({ }^{3} 6_{4,2}\right)=\left(1-25 w^{2}\right)\left(1-w^{2}+16 w^{4}\right), \\
& P\left({ }^{3} 7_{5,2}\right)=\left(1-10 w+35 w^{2}-51 w^{3}+21 w^{4}-4 w^{5}\right), \\
& P\left({ }^{3} 7_{3,4}\right)=\left(1+7 w+26 w^{2}+7 w^{3}+4 w^{4}\right), \\
& P\left({ }^{3} 7_{1,6}\right)=\left(1+12 w+54 w^{2}+112 w^{3}+105 w^{4}+35 w^{5}+4 w^{6}\right), \\
& P\left({ }^{3} 8_{6,2}\right)=\left(1-16 w^{2}+100 w^{4}\right)\left(1-20 w^{2}+16 w^{4}-16 w^{6}\right), \\
& P\left({ }^{3} 8_{4,4}\right)=\left(1+2 w^{2}\right) .
\end{aligned}
$$

Case 4. $f_{i} \neq 0, \cos \phi_{a}$ and $\cos \phi_{b}$ distinct.

$$
\begin{aligned}
& P\left({ }^{4} 3_{2,1}\right)=1, \quad P\left({ }^{4} 4_{3,1}\right)=1, \quad P\left({ }^{4} 4_{2,2}\right)=1, \\
& P\left({ }^{4} 5_{4,1}\right)=\left(1-w-3 w^{2}+4 w^{3}\right),
\end{aligned}
$$

singularities of the associated ODE is that they are also sufficient and each singularity occurs on some Riemann sheet of the integral. 


$$
\begin{aligned}
& P\left({ }^{4} 5_{3,2}\right)=\left(1+4 w+8 w^{2}\right), \\
& P\left({ }^{4} 6_{5,1}\right)=\left(1-10 w^{2}+29 w^{4}\right), \\
& P\left({ }^{4} 6_{4,2}\right)=1, \\
& P\left({ }^{4} 6_{3,3}\right)=1, \\
& P\left({ }^{4} 7_{6,1}\right)=\left(1-3 w-10 w^{2}+35 w^{3}+5 w^{4}-62 w^{5}+17 w^{6}+32 w^{7}-16 w^{8}\right), \\
& P\left({ }^{4} 7_{5,2}\right)=\left(1+8 w+15 w^{2}-21 w^{3}-60 w^{4}+16 w^{5}+96 w^{6}+64 w^{7}\right), \\
& P\left({ }^{4} 7_{4,3}\right)=\left(1-4 w-16 w^{2}-48 w^{3}+32 w^{4}-128 w^{5}\right), \\
& P\left({ }^{4} 8_{7,1}\right)=\left(1-26 w^{2}+242 w^{4}-960 w^{6}+1685 w^{8}-1138 w^{10}\right), \\
& P\left({ }^{4} 8_{6,2}\right)=\left(1-10 w^{2}+32 w^{4}\right), \\
& P\left({ }^{4} 8_{5,3}\right)=\left(1-30 w^{2}+56 w^{4}-1312 w^{6}\right), \\
& P\left({ }^{4} 8_{4,4}\right)=1 .
\end{aligned}
$$

\section{Appendix D. The one term Fermionic toy model}

Here we consider the question of which integral singularities might arise from combinations of $x_{i} x_{j}=1, f_{i}=0$ and $\prod x_{i}=1$.

The analysis of $\tilde{\chi}^{(n)}$ with the full Fermionic factor $\left(G^{(n)}\right)^{2}$ is complicated in large part because of the very many different $x_{i} x_{j}=1$ combinations to consider. Thus we are motivated to look at simpler integrals intermediate between $\Phi_{H}^{(n)}$ and $\tilde{\chi}^{(n)}$, but which lead to the same singularities seen in the ODE for $\tilde{\chi}^{(5)}$ and $\tilde{\chi}^{(6)}$. The simplest situation is where the full Fermionic factor is replaced, for $n$ even, by $\left(h_{12}\right)^{2}$, and for $n$ odd by $\left(f_{12}\right)^{2}$, where [3]:

$$
f_{12}=\frac{1}{2}\left(\sin \phi_{1}-\sin \phi_{2}\right)\left(1+x_{1} x_{2}\right) /\left(1-x_{1} x_{2}\right) .
$$

The integrands in these cases are simple enough that long series can be derived with only slightly more effort than for $\Phi_{H}^{(n)}$. We find, for example, by a mod prime analysis of the ODE for this "one term" reduction of $\tilde{\chi}^{(5)}$, that the head polynomial has the $(1-2 w)$ factor that is in the $\tilde{\chi}^{(5)} \mathrm{ODE}$ and in addition has the factor $\left(1-8 w^{2}\right)$. The latter also shows up in the "one-term" reduction of $\tilde{\chi}^{(6)}$, where it might have been expected since that it is what we found for the $\tilde{\chi}^{(6)}$ ODE by diff-Padé analysis. Explicit solution of the ODE shows the associated singular functions have, as leading terms, $(1-2 w)^{1 / 2}, \ln (1-\sqrt{8} w)$ and $\ln (1+\sqrt{8} w)$ for the "one term" $\tilde{\chi}^{(5)}$ and $\left(1-8 w^{2}\right) \ln \left(1-8 w^{2}\right)$ for the "one term" $\tilde{\chi}^{(6)}$. The observed singularities of the ODE make the simple "one term" reductions of $\tilde{\chi}^{(n)}$ ideal integrals for a Landau singularity analysis and the details of this analysis is the content of the rest of this appendix. As in Appendix B, we allow for various analytic continuations of the integrands. Yet in spite of this we find that the Landau conditions are never satisfied at either $1-2 w=0$ or $1-8 w^{2}=0$. Since the Landau conditions are necessary for the integral to be singular we must conclude that the linear ODE generated from the series have additional singularities not possessed by the integrals.

The addition of the new singularity $x_{1} x_{2}=1$ requires an analysis that can be broken into two parts. The simplest is the determination of the integral singularities

9 "Various" includes all possible sign combinations of the square root factors in the integrand and that means we are considering every possible local environment in the Landau analysis. The same local conditions can differ in the global behaviour of the contour distortions used in the integrals but this does not affect the singularity conditions. In this sense our singularity search is exhaustive. 
arising out of just $x_{1} x_{2}=1$ and $f_{i}=0$. The more involved investigation is for the combination of $f_{i}=0$ with $x_{1} x_{2}=1$ and $\prod x_{i}=1$ simultaneously satisfied and with the normals to the latter two hypersurfaces constrained to be parallel†.

For the simple case of $x_{1} x_{2}=1$ and $f_{i}=0$ for all $i$ we first observe that the $f_{i}=0$ pinch conditions lead to the Case 3 singularities (B.10) and the only new investigation to be done is to determine whether the presence of different integrand factors changes the even $k$ condition in (B.10). The previous proof that the presence of $\left(G^{(n)}\right)^{2}$ did not change the even $k$ condition in (B.10) relied on the fact that $G^{(n)}$ is a product of $h_{i j}$ factors and thus was in essence also a proof for any single $\left(h_{12}\right)^{2}$. Essentially the same proof will apply to $\left(f_{12}\right)^{2}$ provided we take for $f_{i j}$ the original $f_{i j}=1 / 2\left(\sin \left(\phi_{i}\right)-\sin \left(\phi_{j}\right)\right)\left(1+x_{i} x_{j}\right) /\left(1-x_{i} x_{j}\right)$ (see (4) in [3]) because this form can be reduced to a Taylor expansion with terms $\left(\zeta_{i}\right)^{p_{i}}\left(\zeta_{j}\right)^{p_{j}}$ with $p_{i}+p_{j}$ odd exactly as in the argument for $h_{i j}$ following (B.11). The cumulant reduced form $f_{i j}=\left(\sin \left(\phi_{i}\right)-\sin \left(\phi_{j}\right)\right) x_{i} x_{j} /\left(1-x_{i} x_{j}\right)$, which is more convenient for calculating the full $\tilde{\chi}^{(2 n+1)}$, when expanded has terms of both odd and even parity and thus will also generate odd $k$ singularities (B.10). This has been observed and is an additional confirmation of the argument that it is the very special nature of the integrand of the Ising $\tilde{\chi}^{(n)}$ that is responsible for the even $k$ condition in (B.10). It is to be understood in the following that we will be using the original $f_{i j}$ and thus that (B.10) will not be supplemented with odd $k$ terms.

We must also deal with the van Hove singularities arising from $x_{1} x_{2}=1$ with $x_{1} x_{2}$ stationary. The product $x_{1} x_{2}=1$ requires $\zeta_{1}=-\zeta_{2}$ and hence $\cos \left(\zeta_{1}\right)=\cos \left(\zeta_{2}\right)$. This in turn, because of (B.7), gives $\cos \left(\phi_{1}\right)=\cos \left(\phi_{2}\right)$ and $\phi_{1}= \pm \phi_{2}$. The stationary condition is $\sin \left(\phi_{1}\right)=\sin \left(\phi_{2}\right)=0$, which combined with the preceding $\phi_{1}= \pm \phi_{2}$ allows as possible $\phi_{1}, \phi_{2}$ combinations 0,0 and $\pi, \pi$. The corresponding $\cos \left(\zeta_{1}\right)$ and $\cos \left(\zeta_{2}\right)$ are both either $1 /(2 w)-1$ or $1 /(2 w)+1$ so that the equality $\cos \left(\zeta_{1}\right)=\cos \left(\zeta_{2}\right)$ required for $x_{1} x_{2}=1$ is automatic and gives no constraint on $w$. The absence of a first order stationary constraint requires that we go to second order so that in addition to $\zeta_{1}^{\prime}=-\sin \left(\phi_{1}\right) / \sin \left(\zeta_{1}\right)=0$ we demand $\zeta_{1}^{\prime \prime}=-\left(\cos \left(\phi_{1}\right) \sin ^{2}\left(\zeta_{1}\right)+\cos \left(\zeta_{1}\right) \sin ^{2}\left(\phi_{1}\right)\right) / \sin ^{3}\left(\zeta_{1}\right)=0$ and similarly for $\zeta_{2}^{\prime \prime}$. The expression in braces factorizes into $\left(\cos \left(\phi_{1}\right)+\cos \left(\zeta_{1}\right)\right)\left(1-\cos \left(\phi_{1}\right) \cos \left(\zeta_{1}\right)\right)$, which, given (B.7) and the possible values $\cos \left(\phi_{1}\right)= \pm 1$, reduces to $1 / w(1 \pm 1 /(4 w))$. Thus from $\zeta_{1}^{\prime \prime}=0$ we get as the only possible Landau singularities $w= \pm 1 / 4$ or $\infty$, the Case 1 singularities we are not considering.

We begin the analysis where $x_{1} x_{2}=1$ and $\prod x_{i}=1$ are simultaneously satisfied and these hypersurfaces touch tangentially, by deducing the necessary constraint conditions. As before we treat $\phi_{i}, i=1, \cdots, n-1$ as independent giving $\zeta_{i}=\zeta_{i}\left(\phi_{i}\right)$, $i=1, \cdots, n-1$, and $\zeta_{n}=\zeta_{n}\left(\phi_{n}\right)=\zeta_{n}\left(2 \pi k-\phi_{1}-\cdots \phi_{n-1}\right)$. Parallel normals requires $\alpha \partial\left(\zeta_{1}+\cdots+\zeta_{n}\right) / \partial \phi_{i}=\beta \partial\left(\zeta_{1}+\zeta_{2}\right) / \partial \phi_{i}, i=1, \cdots, n-1$, with both $\alpha$ and $\beta$ non-zero. Explicitly,

$$
\begin{array}{ll}
\alpha \cdot\left(\zeta_{1}^{\prime}-\zeta_{n}^{\prime}\right)=\beta \cdot \zeta_{1}^{\prime}, & \alpha \cdot\left(\zeta_{2}^{\prime}-\zeta_{n}^{\prime}\right)=\beta \cdot \zeta_{2}^{\prime}, \\
\alpha \cdot\left(\zeta_{i}^{\prime}-\zeta_{n}^{\prime}\right)=0, & 3 \leq i \leq n
\end{array}
$$

where again $\zeta^{\prime}=\partial \zeta / \partial \phi=-\sin (\phi) / \sin (\zeta)$. Conditions (D.2) imply either $\zeta_{n}^{\prime}=0$

$\dagger$ We need not consider the third possibility, $x_{1} x_{2}=1$ and $\prod x_{i}=1$, satisfied with $x_{1} x_{2}$ and $\prod x_{i}$ simultaneously stationary. The constraints when $x_{1} x_{2}$ and $\prod x_{i}$ are treated as independent are either compatible with each other or not. If the constraints are incompatible solutions will have to be dropped, otherwise they can be kept. In either case there is no possibility of new solutions being generated. 
or $\zeta_{1}^{\prime}=\zeta_{2}^{\prime}$ while conditions (D.3) reduce to the previous stationary conditions (B.5) except that $i$ and $j$ are restricted by $i, j>2$. The new structure of the stationary conditions has implications for the pinch singularities also. Whereas in the discussion at the end of Case 3 we noted that pinch and van Hove type singularity conditions could not mix, here we have the possibility that with $m=2$ in that discussion $\alpha \partial\left(\zeta_{1}+\cdots+\zeta_{n}\right) / \partial \zeta_{1}=\beta \partial\left(\zeta_{1}+\zeta_{2}\right) / \partial \zeta_{1}$ and $\alpha \partial\left(\zeta_{1}+\cdots+\zeta_{n}\right) / \partial \zeta_{2}$ $=\beta \partial\left(\zeta_{1}+\zeta_{2}\right) / \partial \zeta_{2}$ can both be satisfied with $\alpha=\beta$. Thus it is possible to have $\sin \left(\zeta_{1}\right)=\sin \left(\zeta_{2}\right)=0$ in conjunction with the conditions (D.3) that arise out of the remaining derivatives with respect to $\phi_{i}, i>2$. The reverse situation in which $\sin \left(\zeta_{1}\right) \neq 0, \sin \left(\zeta_{2}\right) \neq 0$ and $\sin \left(\zeta_{i}\right)=0, i>2$, is not possible because there is no analogue of the $\alpha=\beta$ solution in this case. In summary, all possibilities covered by (D.2, D.3) plus the allowed pinch situations are given by

$$
\begin{aligned}
& \sin \left(\phi_{n}\right) \cdot\left(\sin \left(\zeta_{1}\right) \sin \left(\phi_{2}\right)-\sin \left(\phi_{1}\right) \sin \left(\zeta_{2}\right)\right)=0, \\
& \sin \left(\zeta_{i}\right) \cdot \sin \left(\phi_{j}\right)=\sin \left(\phi_{i}\right) \cdot \sin \left(\zeta_{j}\right), \\
& i \neq j>2, \quad\left(\sin \left(\zeta_{i}\right) \neq 0, i>2\right)
\end{aligned}
$$

Details for the various alternatives are given below with the major categories a) through d) being the distinctions allowed by (D.4).

a) Pinch case: $\sin \left(\zeta_{1}\right)=\sin \left(\zeta_{2}\right)=0$.

Allowed combinations of $\zeta_{1}, \zeta_{2}$ satisfying $\sin \left(\zeta_{1}\right)=\sin \left(\zeta_{2}\right)=0$ and $x_{1} x_{2}=1$ are 0,0 and $\pi, \pi$. The corresponding $\cos \left(\phi_{1}\right)$ and $\cos \left(\phi_{2}\right)$ are either both $\cos \left(\phi^{(-)}\right)=$ $1 /(2 w)-1$ or both $\cos \left(\phi^{(+)}\right)=1 /(2 w)+1$. If $\phi_{1}=-\phi_{2}$ then conditions (D.5) together with (B.4) are just the conditions for the singularities of $\tilde{\chi}^{(n-2)}$ in the absence of the Fermionic factor $\left(G^{(n)}\right)^{2}$ and have already been discussed. If $\phi_{1}=\phi_{2}$ the sum on phases $\phi_{i}, i>2$, is no longer 0 , but $\pm 2 \phi_{1} \bmod 2 \pi$ with $\phi_{1}$ either $\phi^{(-)}$or $\phi^{(+)}$. For the new singularities that arise we introduce further subdivisions depending on the form taken by (D.5).

For $i>2$, all $\zeta_{i}$ equal and all $\phi_{i}$ equal as the analogue of Case 2. The first new situation arises for $\tilde{\chi}^{(5)}$. Here we set $\zeta_{i}=2 \pi / 3$ and solve $T_{2}\left(\phi^{( \pm)}\right)=$ $T_{3}(1 /(2 w)+1 / 2)$. For $\tilde{\chi}^{(6)}$ set $\zeta_{i}=\pi / 2$ and solve $T_{2}\left(\phi^{( \pm)}\right)=T_{4}(1 /(2 w))$. These are the only possibilities for $n<7$ and the resulting polynomial equations are

$$
\begin{array}{llll}
\left(1+2 w+4 w^{2}-4 w^{3}\right)\left(1+2 w-4 w^{2}-4 w^{3}\right)=0, & \text { for } & \tilde{\chi}^{(5)}, & \text { and } \\
\left(1-w^{2}\right)\left(1-9 w^{2}+16 w^{4}\right)=0, & \text { for } \tilde{\chi}^{(6)} . & & \text { (D. } 6)
\end{array}
$$

For $i>2, \zeta_{i}=\zeta_{a}$ or $\zeta_{b}$ and $\phi_{i}=\phi_{a}$ or $\phi_{b}$ as the analogue of Case 4. The reduction of the singularity conditions to polynomial form is similar to that described in the Case 4 analysis and will not be described further. The only possibilities for $n<7$ are

$$
\begin{aligned}
& \left(1-2 w-4 w^{2}+12 w^{3}+16 w^{4}\right)\left(1-w+9 w^{2}-24 w^{3}+16 w^{4}\right) \\
& \left(1-w+w^{2}+80 w^{3}+352 w^{4}+512 w^{5}+256 w^{6}\right) \\
& \left(1+2 w-20 w^{2}-68 w^{3}-32 w^{4}-64 w^{5}\right)=0, \quad \text { for } \quad \tilde{\chi}^{(5)}, \quad \text { and } \\
& \left(1-w^{2}+16 w^{4}\right)\left(1-41 w^{2}+640 w^{4}-4096 w^{6}\right) \\
& \left(16-664 w^{2}+11273 w^{4}-68290 w^{6}+141889 w^{8}-16896 w^{10}+65536 w^{12}\right) \\
& \left(1-77 w^{2}+1898 w^{4}-20282 w^{6}+107013 w^{8}-160553 w^{10}+198432 w^{12}\right. \\
& \left.-87776 w^{14}-7680 w^{16}+20224 w^{18}+4096 w^{20}\right)=0, \quad \text { for } \tilde{\chi}^{(6)} .
\end{aligned}
$$


Two of the singularity polynomials for $\tilde{\chi}^{(6)}, 1-w^{2}$ and $1-w^{2}+16 w^{4}$, are present also in the absence of $\left(G^{(n)}\right)^{2}$. All other polynomials in (D.6) and (D.7) are new but have not been seen in any ODE analysis. Because the integrand singularity surfaces $x_{1} x_{2}=1$ and $\prod x_{i}=1$ involve the common factor $x_{1} x_{2}$ it is conceivable that the $\phi_{1}$ and $\phi_{2}$ integration contours might always be constrained to lie on the same side of the two surfaces and never be pinched in between. This would explain the absence of these singularities but to resolve such a complicated topological question is not something we have attempted. On the other hand, the absence of these singularities is confirmed by the $\zeta \leftrightarrow \phi$ interchange symmetry discussed in the Case 3 section and the following b) results.

b) van Hove case: $\sin \left(\phi_{1}\right)=\sin \left(\phi_{2}\right)=0$.

Only the $\phi_{1}, \phi_{2}$ combinations 0,0 and $\pi, \pi$ are allowed since the $x_{1} x_{2}=1$ constraint requires $\zeta_{1}=-\zeta_{2}$ and this is only possible with $\cos \left(\zeta_{1}\right)$ and $\cos \left(\zeta_{2}\right)$ either both $1 /(2 w)-1$ or $1 /(2 w)+1$. This is analogous to the $\phi_{1}=-\phi_{2}$ situation of case a) which is that the remaining conditions (D.5) and (B.4) just yield the singularities of $\tilde{\chi}^{(n-2)}$ without $\left(G^{(n)}\right)^{2}$. The difference between what we have here and in a) is that there are no other possibilities: singularities such as (D.6, D.7) are not generated here and, by inference from the $\zeta \leftrightarrow \phi$ interchange symmetry, are not present in a).

c) The combination $\phi_{1}=-\phi_{2}$ and $\zeta_{1}=-\zeta_{2}$ with $\sin \left(\phi_{1}\right) \neq 0, \sin \left(\zeta_{1}\right) \neq 0$.

The $\zeta_{1}=-\zeta_{2}$ condition is required by $x_{1} x_{2}=1$. The remaining $\phi_{1}=-\phi_{2}$ by $\sin \left(\zeta_{1}\right) \cdot \sin \left(\phi_{2}\right)-\sin \left(\phi_{1}\right) \cdot \sin \left(\zeta_{2}\right)=0$ in (D.4). The conditions (D.5) and (B.4) give the singularities of $\tilde{\chi}^{(n-2)}$ without $\left(G^{(n)}\right)^{2}$ as in b).

d) The case $\sin \left(\phi_{n}\right)=0$.

The conditions (D.5) require, for all $i>2, \sin \left(\phi_{i}\right)=0$ and thus $\phi_{i}=0$ or $\pi$. The corresponding $\cos \left(\zeta_{i}\right)$ are $\cos \left(\zeta^{( \pm)}\right)=1 /(2 w) \pm 1$. The condition $x_{1} x_{2}=1$ requires $\zeta_{1}=-\zeta_{2}$ or $\cos \left(\zeta_{1}\right)=\cos \left(\zeta_{2}\right)$ and, then, from the definition (B.7) that $\cos \left(\phi_{1}\right)=\cos \left(\phi_{2}\right)$, or $\phi_{1}= \pm \phi_{2}$. Note that $\phi_{1}=\phi_{2}$ is allowed because (D.4) is no longer a constraint on $\phi_{1}$ or $\phi_{2}$ once $\sin \left(\phi_{n}\right)=0$. In the case that $\phi_{1}=-\phi_{2}$ the phase constraint $\sum \phi_{i}=0 \bmod 2 \pi$ is satisfied if there are an even number of $\phi_{i}=\pi, i>2$, terms. In the $\phi_{1}=\phi_{2}$ case we can set $\phi_{1}=\phi_{2}=\pi / 2$ and thus accommodate an odd number of $\phi_{i}=\pi, i>2$, terms as well. The final result is that when $\sin \left(\phi_{n}\right)=0$, the van Hove singularities are the Case 3 singularities (B.10) for $\tilde{\chi}^{(n-2)}$ but with odd $k$ allowed and when supplemented by Case 5 are:

$$
\begin{aligned}
& T_{k}(1 / 2 w+1)=\quad T_{n-2 m-k}(1 / 2 w-1), \\
& 0 \leq k \leq n-2 m, \quad m=1, \cdots,[n / 2] .
\end{aligned}
$$

The situation here has parallels to that in a) and b). First, the odd $k$ polynomials in (D.8) are not seen in any ODE analysis and the absence of the odd $k$ singularities (D.8) might well have the same explanation as that suggested for the absence of (D.6) in a). Second, recall the remarks just preceding (D.4) that the mixed van Hove/pinch situation in which $\sin \left(\zeta_{1}\right) \neq 0, \sin \left(\zeta_{2}\right) \neq 0$ and $\sin \left(\zeta_{i}\right)=0, i>2$, is not possible and hence yields no singularities. But with the interchange $\zeta \leftrightarrow \phi$, we get exactly the situation described by (D.8) for $k$ odd and thus the absence of these singularities is also confirmed.

The four cases a)-d) exhaust all possibilities for singularities of the toy $\tilde{\chi}^{(n)}$ integrals where a single $\left(f_{12}\right)^{2}$ or $\left(h_{12}\right)^{2}$ replaces the Fermionic $\left(G^{(n)}\right)^{2}$ factor in the integrand. Furthermore, by performing the Landau analysis of the $(n-1)$ dimensional integral analogs of $(5)$ rather that the $(2 n-2)$ dimensional integrals as in [14] we gain 
a powerful symmetry related tool that enables us to compare results obtained in two different ways. Without this tool we can only conclude that the Landau singularity conditions (D.6-D.8) are necessary but not sufficient. With this tool we can eliminate (D.6-D.8) as singularities of the integrals. This proves the toy $\tilde{\chi}^{(n)}$ integrals with $\left(f_{12}\right)^{2}$ or $\left(h_{12}\right)^{2}$ have the same singularities as those without these integrand factors.

We have not investigated systematically what happens when more terms from $\left(G^{(n)}\right)^{2}$ are included but such calculations will have many similarities to what has been done in the case of just $\left(f_{12}\right)^{2}$ or $\left(h_{12}\right)^{2}$. Furthermore, we have not found any obvious candidates for new singularities. Thus we conjecture that the singularities

of the integrals $\tilde{\chi}^{(n)}$ are exactly those of the integrals without the Fermionic $\left(G^{(n)}\right)^{2}$ factor and the complete list of these is the list we have given in Appendix C.

\section{Appendix E. Singularity exponents at Landau singularities}

To determine the singular behaviour of $\tilde{\chi}^{(n)}$ at Landau singularities requires only a local analysis and calculations similar to those already done for the Case 2 circle singularities [3, 4]. If only leading exponent information is of interest, the calculations simplify dramatically and essentially reduce to a power counting argument. We sketch in this appendix the calculations for the cases most analogous to Case 2 and derive the formula for the exponent

$$
p=\left[(n-m)^{2}+m^{2}-3\right] / 2,0 \leq m \leq n,
$$

applicable in many situations. Here $n$ is either the $\tilde{\chi}^{(n)}$ index in the irreducible Cases $2-4$ or the corresponding index of the $\tilde{\chi}$ subgraph in the reducible Case 5 . The $m$ in (E.1) is either the $k$ or $n-k$ as defined in (B.10) and (B.16). These indices are given as subscripts in our polynomial labeling scheme (cf. Appendix C) to allow a direct calculation of $p$ using (E.1). For example, $p=\left(3^{2}+2^{2}-3\right) / 2=5$ for polynomial $\left({ }^{3} 5_{3,2}\right)$ or any of the reducible polynomials $\left({ }^{5} N /{ }^{3} 5_{3,2}\right)$ with $N=5+2 i, i>0$. Note that Case 2 polynomials $\left({ }^{2} n\right)=\left({ }^{2} n_{n, 0}\right)$ have $m=0$ so that (E.1) becomes $p=\left(n^{2}-3\right) / 2$, the formula for the circle singularities derived in $[3,4]$ as exponents satisfying (E.1), which we will refer to as "normal" exponents. We also choose to call the principal disc physical exponents $p=-1$ at the ferromagnetic point and $p=0$ at the antiferromagnetic point "normal". There are other situations, possibly arising from the cancellation in certain expressions after analytic continuation onto different Riemann sheets. Because of our lack of understanding in most of these cases we will simply call the other exponents "anomalous". The following is then to be understood as a very tentative approach to the singularity exponent problem given that we do not have simple general criteria necessary for the "normal" situation to occur. We are in this respect ultimately guided by the agreement or disagreement with the numerical work of Sections 4 and 5. In this regard note that (E.1) represents the leading exponent at a singularity and the derivation below does not exclude exponents greater than $p$. On the other hand, if an exponent less than $p$ is observed a different mechanism is required. An alternative explanation is that the observed exponent is associated with a solution of the ODE that is not the integral $\tilde{\chi}^{(n)}$.

The power counting analysis requires that we distinguish between the two cases $\sin \left(\zeta_{i}\right)=\mathrm{i} \cdot f_{i}=0$ or $\neq 0$. We begin with the latter and determine first the singularities of $\tilde{\chi}^{(n)}$ without the Fermionic factor. The singularities in this case come from the zeros of $1-\prod x_{i}=1-\exp \left(\mathrm{i} \sum \zeta_{i}\right)$. For the behaviour of the integrand in the vicinity of a zero we define $\epsilon$ as the deviation of $\omega-1$ from the Landau singularity value and $\delta \phi_{i}$ as the 
deviation of the corresponding phase values. A Taylor expansion of $\sum \zeta_{i}$ yields $\sum \zeta_{i}=$ $2 \pi m+\sum \delta \phi_{i}\left(\partial \zeta_{i} / \partial \phi_{i}\right)-\epsilon / 2 \sum 1 / \sin \left(\zeta_{i}\right)-1 /(4 \omega) \sum \delta \phi_{i}^{2}\left(1-\cos \zeta_{i} \cos \phi_{i}\right) / \sin ^{3} \zeta_{i}+\ldots$ and the stationary condition (B.5) that $\partial \zeta_{i} / \partial \phi_{i}$ is the same for all $i=1 \ldots n$ implies that the linear term is proportional to $\sum \delta \phi_{i}$ and thus vanishes because of the phase constraint in (9). We conclude that the leading terms in $1-\prod x_{i}$ are of the form $A \epsilon+\sum a_{i} \delta \phi_{i}^{2}$ with $A$ and $a_{i}$ constants. The rescaling $\delta \phi_{i}=\delta \psi_{i} \sqrt{\epsilon}$ puts this denominator factor in the $\tilde{\chi}^{(n)}$ integral into homogeneous form proportional to $\epsilon$. The Jacobian of the transformation from $n-1$ variables $\delta \phi$ to $\delta \psi$ yields another overall factor of $\epsilon^{(n-1) / 2}$ so that the final scaling of the integral singularity is $\epsilon^{(n-3) / 2}$, that is to say, a singularity power $p=(n-3) / 2$ which is exactly that observed in all ODE analyses of these integrals.

The inclusion of the Fermionic factor leads to extra powers of $\epsilon$ that can be determined by counting as follows. In the Case 2 situation treated in [3, 4], there are $n(n-1) / 2$ factors of $h_{i j}^{2}$, each of which has an $\mathrm{O}(1)$ denominator and a numerator proportional to $\left(\delta \phi_{i}-\delta \phi_{j}\right)^{2}$ or $\epsilon\left(\delta \psi_{i}-\delta \psi_{j}\right)^{2}$ and thus $\mathrm{O}(\epsilon)$. The total singularity power then becomes $p=(n-3) / 2+n(n-1) / 2=\left(n^{2}-3\right) / 2$. For Case 4 , to which the present argument applies, there are $k$ phases $\phi_{a}$ and $(n-k)$ phases $\phi_{b}$ at a Landau singularity. This implies that there are now $k(n-k)$ factors of $h_{i j}^{2}$ with numerators close to $\sin ^{2}\left(\left(\phi_{a}-\phi_{b}\right) / 2\right)$ and these are $\mathrm{O}(1)$ and not $\mathrm{O}(\epsilon)$. Only the $k(k-1) / 2$ and $(n-k)(n-k-1) / 2$ factors within each $\phi_{a}$ and $\phi_{b}$ set respectively are $\mathrm{O}(\epsilon)$. This gives the singularity power now as $p=(n-3) / 2+k(k-1) / 2+(n-k)(n-k-1) / 2$ which is (E.1) with $m=k$.

For the reducible Case 5 a new situation arises in the Fermionic factor. Suppose, for example, in our singularity list of $(\phi, \zeta)$ values there are $n-k$ pairs of type $\left(\phi_{b}, \zeta_{b}\right)$ but that of the remaining $k$ pairs, one is $\left(-\phi_{a},-\zeta_{a}\right)$ and only $k-1$ are $\left(\phi_{a}, \zeta_{a}\right)$. Then we know there will be $k-1$ factors of $h_{i j}^{2}$ involving a $\left(-\phi_{a},-\zeta_{a}\right),\left(\phi_{a}, \zeta_{a}\right)$ combination. The numerator in each of these factors will be close to $\sin ^{2}\left(\phi_{a}\right)$ and thus $\mathrm{O}(1)$ and not proportional to $\left(\delta \phi_{i}-\delta \phi_{j}\right)^{2}$ and $\mathrm{O}(\epsilon)$. The denominator on the other hand is $\left(1-x_{i} x_{j}\right)^{2}=\left[1-\exp \left(i\left(\zeta_{a}+\delta \zeta_{i}\right)+i\left(-\zeta_{a}+\delta \zeta_{j}\right)\right)\right]^{2} \approx-\left(\delta \zeta_{i}+\delta \zeta_{j}\right)^{2}=\mathrm{O}(\epsilon)$. In summary, there are $k-1$ Fermionic factor terms each $\mathrm{O}(1 / \epsilon)$. Furthermore, of the original $k(k-1) / 2$ factors involving factors of $\phi$ of type $\phi_{a}$ only the remaining $k(k-1) / 2-(k-1)=(k-1)(k-2) / 2$ are $\mathrm{O}(\epsilon)$ as before. Factors involving only type $\phi_{b}$ or type $\pm \phi_{a}$ and $\phi_{b}$ combinations are also unaffected. Counting all powers of $\epsilon$ listed above shows that the singularity exponent will be $(n-3) / 2-(k-1)+$ $(k-1)(k-2) / 2+(n-k)(n-k-1) / 2=\left[(k-2)^{2}+(n-k)^{2}-3\right] / 2$ which is again (E.1) but with $m$ and $n-m$ identified with the irreducible subgraph values $k-2$ and $n-k$. If there is more than one sign reversed pair in the reducible Case 5 , then the above counting argument can be repeated and leads to the conclusion that (E.1) is the general result.

The arguments when $\sin \left(\zeta_{i}\right)=\mathrm{i} \cdot f_{i}=0$ at the Landau singularity are very similar to what is described above and show that (E.1) still applies, in particular for Case 3. One technical difference and partial result is worth noting. Each $f_{i}$ is of the form $\sqrt{\left(\epsilon+a_{i} \delta \phi_{i}\right)}$ and thus the appropriate rescaling to make this singular function homogeneous in $\epsilon$ is $\delta \phi_{i}=\delta \psi_{i} \cdot \epsilon$. The singularity power counting in the absence of the Fermionic factor then gives an $\epsilon^{n-1}$ from the Jacobian of the transformation, an $\epsilon^{-n / 2}$ from the $n$ denominator factors $f_{i}$ and another $\epsilon^{-1 / 2}$ from the denominator $1-\prod x_{i}$ which reduces, in leading order, to $\sum f_{i}$. The product of these three $\epsilon$ factors is $\epsilon^{(n-3) / 2}$ and thus we have as the singularity exponent in the absence of the Fermionic 
factor the value $p=(n-3) / 2$ exactly as in the $\sin \left(\zeta_{i}\right)=\mathrm{i} \cdot f_{i} \neq 0$ situation.

One set of comparisons of (E.1) with observed exponent values can be found in Table 4. While there are many cases of agreement, it is also clear that there are "anomalous" exponents that we cannot account for. These include the $-2,-7 / 4,-3 / 2$ and $-5 / 4$ at the ferromagnetic point, and more relevant for the present discussion, the powers $2,5 / 2$ and 0 for the polynomials $\left({ }^{5} 5 /{ }^{2} 3\right)$ and $\left({ }^{5} 5 /{ }^{3} 3_{1,2}\right)$. The understanding of these cases is left as a challenge for the future.

\section{References}

[1] Onsager L 1944 Phys. Rev. 65117

[2] Wu T T, McCoy B M, Tracy C A and Barouch E 1976 Phys. Rev. B 13316

[3] Nickel B 1999 J. Phys. A: Math. Gen. 323889

[4] Nickel B 2000 J. Phys. A: Math. Gen. 331693

[5] Palmer J and Tracy C 1981 Adv. Appl. Math. 2329

[6] Yamada K 1984 Prog. Theor. Phys. 711416

[7] Guttmann A J and Enting I G 1996 Phys. Rev. Lett. 76344

[8] Orrick W P, Nickel B , Guttmann A J and Perk J H H 2001 J. Stat. Phys. 102795 and arXiv: cond-mat/0103074

[9] Boukraa S, Hassani S, Maillard J M, McCoy B M and Zenine N 2007 J. Phys. A: Math. Theor. 408219 and arXiv:math-ph/0703009

[10] Zenine N, Boukraa S, Hassani S and Maillard J M 2004 J. Phys. A: Math. Gen. 379651 and arXiv:math-ph/0407060

[11] Zenine N, Boukraa S, Hassani S and Maillard J M 2005 J. Phys. A: Math. Gen. 38 (2005) 1875 and arXiv:hep-ph/0411051

[12] Zenine N, Boukraa S, Hassani S and Maillard J M 2005 J. Phys. A: Math. Gen. 384149 and arXiv:cond-mat/0502155

[13] Boukraa S, Hassani S, Maillard J M and Zenine N 2007 J. Phys. A: Math. Theor. 402583 and arXiv:math-ph/0701016

[14] Boukraa S, Hassani S, Maillard J M and Zenine N 2007 J. Phys. A: Math. Theor. 4011713 and arXiv:0706.3367

[15] Zenine N, Boukraa S, Hassani S and Maillard J M 2005 J. Phys. A: Math. Gen. 389439 and math-ph/0506065

[16] Nickel B 2005 J. Phys. A: Math. Gen. 384517

[17] McCoy B M and Wu T T 1980 Phys. Rev. Lett. 45675

[18] Perk J H H 1980 Phys. Lett. A 793

[19] Jimbo M and Miwa T 1980 Proc. Japan Acad. A 56405 and Erratum 1981 Proc. Japan Acad. A 57347

[20] Knuth D E 1997 Seminumerical Algorithms. The Art of Computer Programming, Vol 2., 3rd Edition, (Reading, Mass.: Addison Wesley)

[21] http://www.ms.unimelb.edu.au/ iwan/ising/Ising_ser.html

[22] Nappi C R 1978 Nuovo Cim A 44392

[23] Crandall R and Pomerance C 2005 Prime Numbers, A computational perspective, 2nd edition. (New York: Springer)

[24] Guttmann A J and Joyce G S 1972 J. Phys. A: Math. Gen 5 L81

[25] Bailey D H, Borwein J M and Crandall R E 2006 J. Phys. A: Math. Gen 3912271

[26] Rehr J J, Joyce G S and Guttmann A J 1980 J Phys A: Math. Gen. 131587

[27] Tracy C A 1978 Painlevé transcendents and scaling functions of the two-dimensional Ising model, Non-linear Equations in Physics and Mathematics, ed. A.O. Barut, pp. 221-237 (Dordrecht, Holland: D. Reidel Publishing Co.)

[28] van der Waall A 2002 Lamé Equations with Finite Monodromy, Thesis, Utrecht University

[29] Saito T 1958 Math. Sem. Rep. Vol.10 No.3 101

[30] Ince E L 1956 Ordinary Differential Equations (New York: Dover)

[31] van Hove L 1953 Phys. Rev. 891189

[32] Landau L 1959 Nucl. Phys. 13181

[33] Eden R J, Landshoff P V, Olive D I and Polkinghome J C 1966 The Analytic S-Matrix, (Cambridge: Cambridge Univ. Press) 